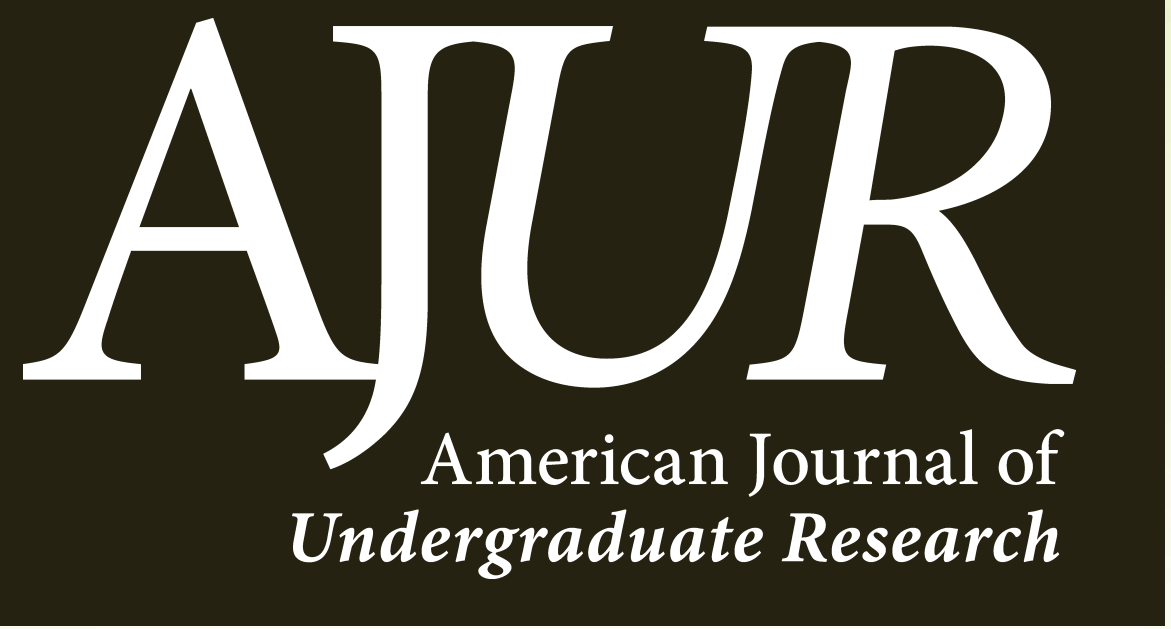

Volume 15 | Issue 2 | September 2018 www.ajuronline.org 


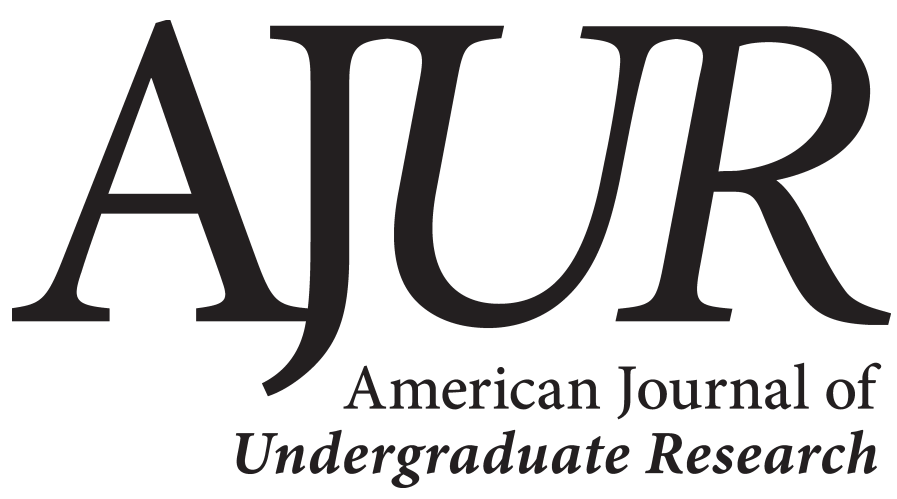

Volume 15 | Issue 2 | September 2018

www.ajuronline.org

AJUR History and Editorial Board

Special Thanks to AJUR's Sponsors

5

Determining Nutritional Barriers to Access to Fresh Fruits, Vegetables, and Whole Grains in Older Americans

Tornia C. Anderson-Morgan, Melissa Fett, Michelle Jasso, Aisha Moten, \& Elgloria Harrison

Minimizing the Cost of Guessing Games

David Clark, Lindsay Crap

Analysis of Fungal Contamination on Commercially Sold Rice in Puerto Rico

Nicole Colón Carrión, Chad Lozada Troche

Characterization of Ambient Particulate Matter Sampled at an Active Sand Mine Facility in Northwestern Wisconsin

Julie Zhang, Callie Fischer, Joe Oster, Gabriel de Carvalho Chaves, Rachel Mooney, \& Patricia Cleary

Dextran Sulfate Based Gel Coating for Drug Eluting Balloon Angioplasty

Jenna Mosier, Hannab Stealey, Kalifa Stringfield, Katie Webb, \& C. LaShan Simpson 
American Journal of Undergraduate Research (AJUR) is a national, peer-reviewed, open-source, quarterly, multidisciplinary student research journal. It is indexed internationally by EBSCO, and is listed via the Library of Congress under ISSNs of 1536-4585 (for print) and 2375-8732 (for web). The journal was established in 2002 and incorporated as a non-for profit organization in 2018.

EDITORIAL TEAM Volume 15 / Issue 2 / September 2018

Dr. Kestutis G. Bendinskas, Executive Editor, editor@ajuronline.org

Dr. Anthony Contento, Copy Editor, Treasurer

Peter Newell, Editor, Secretary

Rose Throop, Print

Daniel Laird, Web Master

EDITORIAL BOARD by subject area

\footnotetext{
ACCOUNTING

Dr. Dean Crawford,

dean.crawford@oswego.edu

ARCHEOLOGY

Dr. Richard Redding,

rredding@umich.eduu

ART HISTORY

Dr. Lisa Seppi,

lisa.seppi@oswego.edu

ASTROPHYSICS

Dr. Shashi Kanbur,

shasbi.kanbur@oswego.edu

BEHAVIORAL NEUROSCIENCE

Dr. Aileen M. Bailey,

ambailey@smcm.edu

BIOCHEMISTRY

Dr. Pamela K. Kerrigan,

pamela.kerrigan@mountsaintvincent.edu

Dr. Nin Dingra,

ndingra@alaska.edu

BIOENGINEERING

Dr. Jorge I. Rodriguez, jorger@clemson.edu

Dr. Jessica Amber Jennings,

jjnnings@memphis.edu

BIOINFORMATICS

Dr. Kevin Daimi,

daimikj@udmercy.edu

Dr. John R. Jungck, jungck@udel.edu

Dr. Isabelle Bichindaritz, ibicbind@oswego.edu

BIOLOGY, PHYSIOLOGY

Dr. David Dunn,

david.dunn@oswego.edu

BIOLOGY, DEVELOPMENTAL

Dr. Poongodi Geetha-Loganathan,

p.geethaloganathan@oswego.edu

BIOLOGY, MICROBIOLOGY

Dr. Peter Newell,

peter.newell@oswego.edu BOTANY

Dr. William R. Bromer, wbromer@stfrancis.edu
}

\author{
ENGINEERING, ENVIRONMEN- \\ TAL \\ Dr. Eileen M. Cashman, \\ eileen.cashman@humboldt.edu \\ ENGINEERING, MANUFACTURING \\ AND CONSTRUCTION, ROBOTICS \\ Dr. Haoyu Wang, \\ wanghao@mail.ccsu.edu \\ ENGINEERING, SOFTWARE$$
\text { Dr. Kevin Daimi, }
$$$$
\text { daimikj@udmercy.edu }
$$ \\ ENVIRONMENTAL SCIENCES \\ Dr. Eileen M. Cashman, \\ eileen.cashman@bumboldt.edu \\ Dr. Christopher Baltus, \\ christopher.baltus@oswego.edu \\ Dr. Mark Baker, \\ mark.baker@oswego.edu \\ MEDICAL SCIENCES \\ Dr. Jessica Amber Jennings \\ jjnnings@memphis.edu \\ METEOROLOGY \\ Dr. Steven Skubis, \\ steven.sk.ubis@oswego.edu \\ MUSIC \\ Dr. Juliet Forshaw, \\ juliet.forshaw@oswego.edu
}

FILM AND MEDIA STUDIES

Dr. Lauren Steimer,

Isteimer@mailbox.sc.edu HISTORY

Dr. Richard Weyhing,

richard.weybing@oswego.edu

Dr. Murat Yasar,

muratyasar@oswego.edu

HONORARY EDITORIAL BOARD MEMBER

Dr. Lorrie Clemo,

lorrie.a.clemo@gmail.com

JURISPRUDENCE

Bill Wickard, Esq,

William.Wickard@KLGates.com

KINESIOLOGY

Dr. David Senchina, david.sencbina@drake.edu

LITERARY STUDIES

Dr. Douglas Guerra,

douglas.guerra@oswego.edu

MATHEMATICS

Dr. John Emert,

emert@bsu.edu

Dr. Jeffrey J. Boats,

boatsjj@udmercy.edu

Dr. J.D. Phillips,

jophilli@nmu.edu

Dr. Dele Oluwade,

deleoluwade@yahoo.com
NANOSCIENCE AND CHEMISTRY

Dr. Gary Baker,

bakergar@missouri.edu

NEUROSCIENCE

Dr. Pamela E. Scott-Johnson, pscottj@calstatela.edu PHYSICS

Dr. Mohammad Islam, mohammad.islam@oswego.edu POLITICAL SCIENCE

Dr. Katia Levintova,

levintoe@uwgb.edu PSYCHOLOGY

Dr. Joseph DW Stephens, jdstephe@ncat.edu

Dr. Melanie Dyan Hetzel-Riggin, mdh33@psu.edu

Dr. Pamela E. Scott-Johnson, pscottj@calstatela.edu

SOCIAL SCIENCES

Dr. Rena Zito, rzito@elon.edu STATISTICS

Dr. Mark Ecker, mark.ecker@uni.edu

TECHNOLOGY, ENGINEERING

Dr. Recayi Pecen, regpecen@na.edu 


\section{SPECIAL THA NKS}

AJUR is made possible through the volunteer efforts of our editorial team and assistance of our sponsors.

Support for this issue has been provided by the Office of the Provost at the State University of New York at Oswego. Thank you!

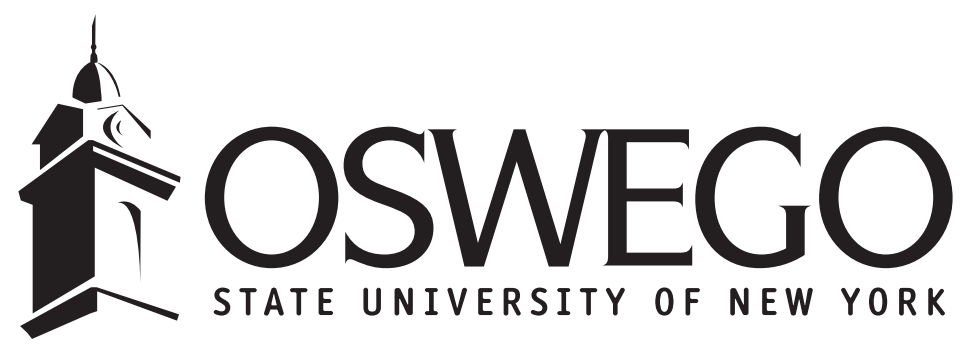

Please support undergraduate research

Request sponsorship information fromeditor@ajuronline.org 


\title{
Determining Nutritional Barriers to Access to Fresh Fruits, Vegetables, and Whole Grains in Older Americans
}

\author{
Tornia C. Anderson-Morgan, Melissa Fett, Michelle Jasso, Aisha Moten, \& Elgloria Harrison \\ Department of Health, Nursing, and Nutrition, University of the District of Columbia, W ashington, D.C. \\ Students: tornia.andersonmorga@udc.edu,melissa.fett@udc.edu,michelle.jasso@udc.edu, aisha.moten@udc.edu \\ Mentor:eharrison@udc.edu*
}

\begin{abstract}
The University of the District of Columbia is a partner of the Northeast Hatch Multistate Research Collaborative. This research project, known as the UDC NE 1439, was designed as a pilot study to determine the barriers that prevented the consumption of fresh fruits, vegetables, and whole grains among older adults in Wards 5, 7, and 8 in the District of Columbia (DC). The residents of these wards have been shown to have higher rates of chronic diseases. Data shows that Ward 7 has the highest rate of deaths due to diabetes. Furthermore, Wards 5, 7, and 8 have the highest percentage of hypertension and diabetes This project used a quantitative and qualitative survey instrument, which included 53 questions and/or statements using a Likert scale: demographics (8 questions); household information (2 questions); shopping habits (8 questions); eating habits, including the identity of the person who prepared meals in the home (21 questions); physical fitness (11 questions); and policy (3 questions). Ninety-six (96) older adults participated in the survey, with a gender distribution of $77 \%$ female (68 individuals), $22 \%$ male ( 24 individuals), and $1 \%$ not reported ( 1 individual). The racial distribution of the participants was 91\% (87) African American, .01\% (one) Asian American, .01\% (one) Caucasian, .01\% (one) Native American, and .03\% (three) others/not reported. Additionally, according to the participants' residency results, Ward 5 accounted for $23 \%$ of the participants (24 participants), Ward 7 for $38 \%$ (33 participants), and Ward 8 for 38\% (33 participants). The participants' average age was $\geq 56$, (45 to 76 and over) and most were the primary financial supporters of their households and the primary grocery shoppers in their families. In conclusion, this project determined that access to fresh fruits and vegetables and travel time to a full-service grocer were less prominent barriers; this was an unexpected finding. Though the participants indicated sufficient access to fresh fruits, vegetables, and whole grains, they lacked nutrition-based knowledge. Many of them viewed unfitting food choices as healthy. An intervention centered on nutrition education using food demonstrations and grocer tours would improve nutrition knowledge in this population. This offered these nutrition and dietetics research assistants with an opportunity to provide nutrition education to the population in question. Moreover, it presents an opportunity to extend nutrition education to all seniors across the Washington, DC region.
\end{abstract}

\section{KEYWORDS}

Fruits; Vegetables; Whole Grains; Urban; Disease; African American; Health; Nutrition; Food Security; Food Desert, Senior Citizens

\section{INTRODUCTION}

Heart disease and stroke are the third and fourth leading causes of death in the United States, and African Americans bear a higher burden of heart disease and stroke than Caucasians., ${ }^{1,2}$ Diabetes mortality rate in Wards 5 (38.2\%), Ward 7 (44.6\%), and Ward $8(31.8 \%)$ as of 2008 and $2009 .{ }^{3}$ The prevalence of End Stage Renal Disease (ESRD) for the District of Columbia in 2010 was 69.6 per 100,000 compared to the United States at 37.54 per 100,000. ${ }^{4}$ In 2015, the prevalence and incident of ESRD were 2429 and 404 per million state residents respectively. ${ }^{3}$ African Americans are likely to consume fewer fruits and vegetables than the United States Department of Agriculture (USDA) dietary recommendations. ${ }^{1}$ The protective benefits of fruits and vegetables have been identified in other studies, which have shown them to reduce the mortality risk that heart disease and stroke pose in minority populations ${ }^{2,6}$. The Sustainable DC plan is the District's vision for improving the health and well-being of its citizens, and it relies on two targeted health goals: Target \#1 involves cutting the citywide obesity rate by $50 \%$ by 2032 ; Target \#2 entails ensuring that, by $2032,75 \%$ of DC residents live within a quarter mile of a community garden, farmers' market, or healthy corner store. ${ }^{7}$ The aim of this research project was to identify the barriers that prevented the consumption of fruits and vegetables mainly among African American communities in Washington, DC, from January 2016 through August 2017. This was because African Americans in the District of Columbia showed a similar disease burden pattern to the national average in the United States where heart disease and stroke were concerned; heart disease and diabetes are the two leading causes of death in this region. The research question that drove this inquiry follows: What are the barriers that prevent the consumption of fresh fruits and vegetables by older adults in Wards 5, 7, and 8 in the District of Columbia? 
Haynes-Maslow et al. identified lack of access to quality food (a shortage of full-service grocery stores), lack of financial resources to purchase high-quality fruits and vegetables, and lack of knowledge of the benefits of fruits and vegetables as barriers to healthy eating behaviors. ${ }^{8}$ The District of Columbia Behavioral Risk Factor Surveillance System (BRFSS) Annual Health Report confirmed that fruit and vegetable consumption were low among African American citizens and were particularly low in Wards 5, 7, and 8 (Wards are administrative divisions within the District of Columbia; each ward represents approximately 75,000 persons). ${ }^{3}$ Given that Wards 5, 7, and 8 have the highest rates of obesity and diabetes and the lowest rates of fruit and vegetable consumption, questions were posed to identify the barriers that prevented this group of mainly African Americans residents from consuming healthy diets of fruits and vegetables. Diets rich in fruits and vegetables are known to reduce the risk of stroke and high blood pressure. ${ }^{2}$ Access to fruits and vegetables while participating in physical activities, has been demonstrated to improve the quality-of-life outcomes of older adults and to reduce risk factors associated with chronic diseases. ${ }^{8}$

It is expected that the older adult population will reach approximately 71 million by the year 2030 and is considered one of the fastest segment of population growth in the United States according to Brewer et al. ${ }^{9}$ Nicklett and Kadell noted that older adults are at a higher risk for chronic conditions such as diabetes, heart disease, and cancer. ${ }^{10}$ Nicklett and Kadell examined the relationship between fruits and vegetable consumption and the subsequent health outcomes and found that older adults tend to eat more fruits and vegetables than younger adults. ${ }^{10}$ Nicklett and Kadell found some of the predictors that influenced the consumption of fruits and vegetables in older adults were 1) health status, 2) geographic /physical environment, 3) demographics, 4)social support, 5)race/ethnicity, 6) socioeconomic status, and 7) dietary knowledge. ${ }^{10}$ Brewer et al. conducted a quasiexperimental pilot study of 35 older adults attending senior centers with 19 in the intervention group and 16 in the control groups to determine actual fruit and vegetable intake. ${ }^{9}$ Brewer et al. found that those in the intervention group significantly increased their fruit and vegetable intakes. This study concluded that a short nutritional educational program was linked to an increase in fruit and vegetable intake. ${ }^{9}$

Interestingly, a 2010 Gallup poll of 176,544 interviews nationwide revealed that access to fresh produce might not be the problem. ${ }^{11}$ This report showed that $92 \%$ of Americans said they had access to fresh fruits and vegetables, yet only half of Americans consumed the recommended five servings of fruits and vegetables per day. ${ }^{11}$ There has been increasing focus on ways of reducing obesity by improving fruit and vegetable intake in African Americans because African Americans are dying at higher rates from conditions that are preventable. Some studies show that the association of African American culture and food choices has become a barrier to understanding healthy food selection and the impact of food choices on the health and well-being of an individual. Understanding African Americans entails understanding their culture, which explains in part why many research programs involving African Americans are framed around faith-based organizations, particularly the African American church. Many research studies in African American communities are designed to shed light on the barriers and on the interventions that have been successful at increasing fruit and vegetable consumption.

\section{Barriers}

Research evidence has acknowledged the existence of many barriers that prevent the consumption of fruits and vegetables in older adults, particularly African Americans. Resnicow et al. noted two barriers that emerged from focus groups: One was that participants were not aware of the serving sizes of fruits and vegetables; the other was that they were unaware of the recommended 5 servings of fruits and vegetables. ${ }^{12}$ In comparison, Lucan, Barg, and Long found that, in a low-income African American community, cost and the participant's ability to afford fruits and vegetables were barriers. ${ }^{13}$ John and Ziebland conducted a 6-month randomized control study of 40 participants in a primary setting and found that children and male partners were barriers to eating fruits and vegetables; however, their research also noted that men with supportive partners increased their consumption of fruits and vegetables. ${ }^{14}$

In addition, John and Ziebland found that cost and travel time were identified as barriers. ${ }^{14}$ Lastly, Stephen $e t$ al. conducted a time period study and measured barriers during two time periods. ${ }^{15}$ They found that the rate of consumption of fruits and vegetables varied. The results suggested that buying fresh fruit every day led to too much waste. ${ }^{9}$ These studies demonstrated that participants who lacked adequate resources and nutritional knowledge and had unsupportive households were more likely to not consume adequate amounts of fruits and vegetables. While these studies provided similar findings on barriers, other studies provided interventions that improved fruit and vegetable consumption.

\section{Review of Previous Interventions}

Pinsker et al. conducted a 12-week community engagement project called Body and Soul to demonstrate healthy recipes and peer counseling in 20 black churches. ${ }^{16}$ The Body and Soul project was described as having four components: pastoral involvement, educational activities (cooking demonstration), a change in church policies (healthy food options at church events), and peer counseling. ${ }^{17}$ While the researchers reported success in the implementation of this project, they also reported implementation challenges such as variations in peer counseling and low turnout at follow-up events. One of the main outcomes of Body and Soul 
was that it proved to be an example of a simple intervention that could be implemented with minimum resources. Pinsker $e t$ al. reported an increase in the consumption of fruit and vegetables from baseline to follow-up. ${ }^{16}$

The GoodNews Trial was a community-based research study intended to reduce the risk of cardiovascular disease in the southernmost part of Dallas, Texas. It was a partnership between researchers and church pastors in 20 black churches to train 20 lay health promoters (LHP), who would recruit church members to participate in the study. ${ }^{18}$ The GoodNews Trial administered a Dietary Health Questionnaire (DHQ), which determined that most of the African Americans in the study consumed less than the recommended daily requirement of fruits and vegetables and that the use of lay health promoters and church pastors was the best way to reach African Americans when promoting healthy lifestyle changes. These studies demonstrated that using black churches to reach their members had many advantages and resulted in some changes in dietary habits.

Satia et al. surveyed 658 African Americans on nutrition label use and found that those who considered themselves healthier had a strong belief in the diet-cancer relationship, were trying to lose weight, and showed higher nutrition label use. ${ }^{19}$ Lewis $\mathrm{et}$ al. conducted a study to assess dietary information use among persons with chronic diseases using a representative sample of the United States population. ${ }^{20}$ They found that those with chronic diseases reported better nutrition awareness than those who did not have chronic diseases; however, this knowledge did not translate into better or improved dietary behaviors. ${ }^{20}$

Wright et al. demonstrated a correlation between diet quality and cognitive performance in the Healthy Aging in Neighborhoods of Diversity across the Lifespan (HANDLS) study. ${ }^{21}$ These researchers found that African Americans and those living in poverty might be at a greater risk for poor cognitive performance than those who had higher-quality diets. Interestingly, the Sustained Attention to Response Task (SART) was linked to mindfulness meditation training and yoga exercises, which have been used in previous studies to correlate aging and loss of cognitive ability. ${ }^{22}$ Sen suggested that SART was linked to mindfulness, meditation training, and yoga exercises, which have been shown to lower blood pressure and relieve stress--known risk factors for Alzheimer's disease and cardiovascular diseases. ${ }^{23}$ Kicklighter et al. followed five African American grandparents who participated in a home-based nutrition and physical activity intervention called "Project Healthy Grandparent Program." 24 The goal of this program was to increase the grandparents' knowledge and ability to select nutritious foods for themselves and their grandchildren. The study showed that one of the most important concerns for these grandparents was how to cook more nutritiously. Higgins and Murray noted that grandparents and others presently caring for grandchildren were more health conscious than when they were first parents; however, most found that eating a healthy nutritious meal posed financial challenges. ${ }^{25}$ This was particularly noted in Kansas, where the number of grandparents raising grandchildren increased to 43 percent from 1990 to 2000 . This was a 13 percent increase over the national average. ${ }^{25}$

Higgins and Murray also pointed out that the presence of grandchildren in the home changed the eating habits of grandparents and grandchildren. ${ }^{25}$ These authors noted that the presence of grandchildren might negatively influence grandparents' ability to make healthy dietary choices owing to the grandchildren's preferences and taste; they also noted that cultural influence might make it more difficult to move away from traditional foods. ${ }^{18}$ Environmental factors, such as lack of accessible and affordable foods, failure to read nutritional labels, raising grandchildren, poor cognitive performance, and traditional foods could be barriers to an African American's consumption of a healthier diet. Finding ways to decrease the impact of these barriers would have health consequences, lowering the risk of heart disease, stroke, and diabetes.

\section{METHODS}

The research project piloted a nutrition barrier survey in Wards 5, 7, and 8 to determine the ease with which residents could access fresh fruits, vegetables, and whole grains. The University of the District of Columbia Institutional Review Board approved the study. The project recruited 96 predominantly African American individuals from Wards 5, 7, and 8 at congregational sites, including faith-based organizations and recreational centers. The participants were informed of the study's purpose and signed letters of consent to participate. The students conducting the surveys received training on conducting human subject research and demonstrated the ability to conduct the survey uniformly. Each of them was accompanied by the principal investigator on the first survey to confirm that the approach to administering the questions was uniform.

\section{Survey Development}

The student research assistants and the principal investigators participated in the design and testing of the survey instrument, which had both closed- and open-ended questions. Several questions used a four-point Likert scale (1: never; 2: seldom; 3: sometimes; and 4: often) to collect relevant data. The survey included 53 questions and/or statements in the following categories: demographics (8 questions); household information (2 questions); shopping habits (8 questions); eating habits, including the identity of the person who prepares meals in the home (21 questions); physical fitness (11 questions); and policy (3 questions). While the survey included 53 questions, quantitative analysis was provided regarding the following: demographics, household information, and shopping habits, which included access to fruits and vegetables. 
Participants were selected via the convenience sampling method. Treadwell noted that convenience sampling was nonprobability sampling; as the name suggests, it was a matter of convenience for the student researchers to encounter older adults who were frequent visitors to meal sites or faith-based organizations within the wards. ${ }^{26}$ Each of the students agreed to work in Ward 5, 7, or 8 and to contact faith-based organizations and churches that participated in providing meals to seniors there. Once approval was received, the principal investigator conducted an overview of the project and introduced the research assistants who would be conducting the surveys. The principal investigator provided training on how to administer the survey and each research assistant was required to complete human subject training on the National Institutes of Health Human Subject Training online web portal prior to the administration of the survey.

\section{Quantitative Approach}

This research had both quantitative and qualitative aspects. The survey instrument represented the quantitative component, and the student observations and discussions with the participants following the survey represented the qualitative component. Each participant who agreed to take the survey could complete it on his or her own or with minimum assistance from the relevant research assistant. The survey was administered in a paper format that required each participant to read each question and place a mark on the response that best represented his or her answer. Participants who experienced reading difficulties asked for assistance, which entailed having the questions read to them. While the questions may have been read to these participants, they could mark their answers independently. Each survey lasted 45 minutes on average, and small tokens of appreciation were distributed after all the surveys were completed.

\section{Qualitative Approach}

In the qualitative component of this research, participants would openly discuss their concerns about their food consumption. The words of the participants were documented in a journal, and what emerged from these discussions were themes that shaped an understanding of the participants' thoughts and beliefs in relation to food. Upon the completion of the surveys, each research assistant remained on site to answer the questions that the participants may have had. The participants were able to reflect on the survey and to articulate their views on what they believed were barriers to their consumption of fresh produce. These conversations came as a surprise to the research assistants as they were not expecting to collect such rich qualitative data. Because the participants were willing to share their thoughts, written notes were taken of these conversations. Typically, in qualitative research, these types of conversations would be considered focus groups and would ordinarily be taped; however, these conversations were not taped. The impromptu conversations were shared with the principal investigator, who required each research assistant to write them in a journal. This was because the conversations set the context for an understanding of how participants viewed their access to fresh produce and what they thought were barriers. The research assistants' advisor noted that these were unexpected jewels that researchers find when they least expect them.

\section{RESULTS}

\section{Quantitative Data}

There were approximately equal percentages of participants from Wards 5, 7, and 8. Figure 1, which presents descriptive data results, shows a total of 96 respondents, of whom $73 \%$ were reported to be female (68) and $26 \%$ were reported to be male (24); for $1 \%$ (1), the gender was not reported. Figure 2 shows the ethnicities of the participants: 87 were African American, one was Asian, one Caucasian, and one Native American; ethnicity was not reported for three others. Figure 3 indicates the wards in which the participants resided. Ward 5 represented $23 \%$ (26 participants), Ward 7 represented $38 \%$ (33 participants), and Ward 8 represented $38 \%$ (33 participants).

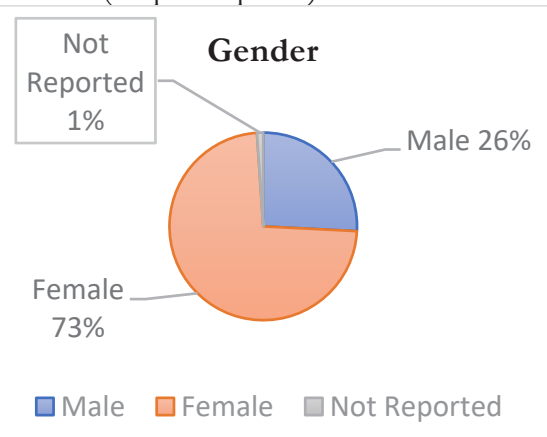

Figure 1. Participants' gender, male, female, and those not reporting.

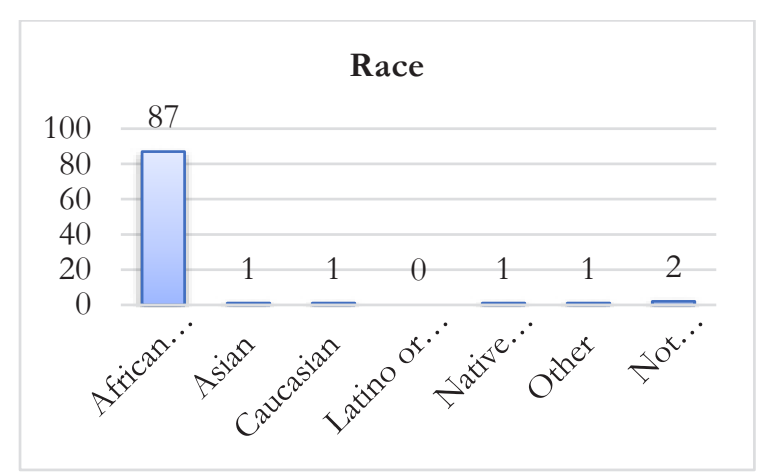

Figure 2. Participants' race for the targeted population for this project. 


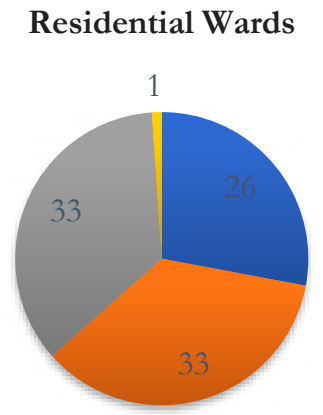

Ward 5 ward 7 ward $8 \quad$ Not reported

Figure 3. Participants' residential ward in Washington, DC.

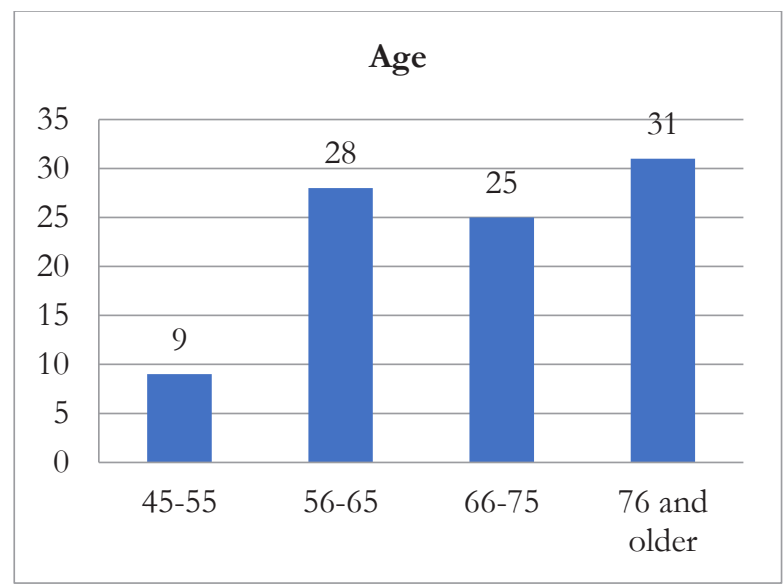

Figure 4. Participants' age for the targeted population for this project.

As per Figure 4, 89\% of the population was over age 56. This was not surprising given the wards and the characteristics of the targeted population. To determine the level of access to fresh fruits, vegetables, and whole grains, the following information was documented: 1) the identity of the individual who was considered the primary financial supporter of the household and 2) the identity of the one who was considered the primary grocery buyer in the family. Figures $\mathbf{5}$ and $\mathbf{6}$ show that many of the female respondents indicated they were the primary financial supporters $(89 \%)$ and the primary grocery shoppers for their households (78\%). When asked about access to fresh fruits, vegetables, and whole grains, $50 \%$ and 32\% of the participants respectively responded, "Often," or "Sometimes," as indicated in Figure 7. Most participants were women, who were primarily responsible for shopping, which meant that women made the primary decision to purchase fruits and vegetables. On the other hand, fewer male respondents indicated they were the primary grocery buyers.

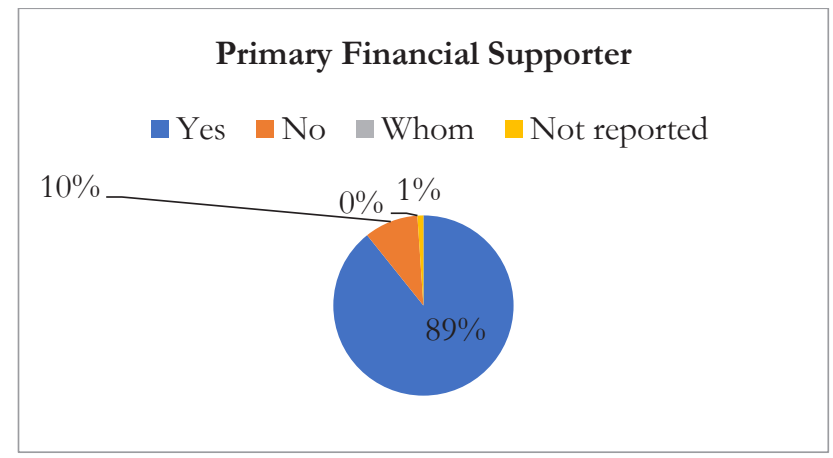

Figure 5. Primary financial supporter for groceries for the family.

\section{Access to Fresh Fruits, Vegetables, Grain \\ Never $\quad$ Seldom $\square$ Sometimes $\square$ Often}

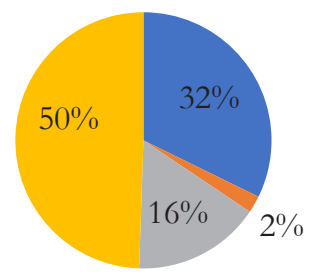

Figure 7. Access to fresh fruits/vegetables/whole grains.

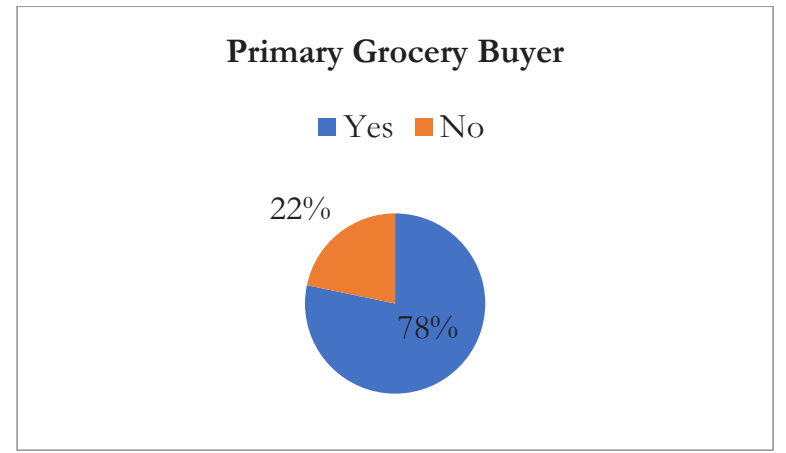

Figure 6. Participants who were the primary grocery buyer.

\section{Use Shopping List}

- Never $\square$ Seldom $\square$ Sometimes $\quad$ Often

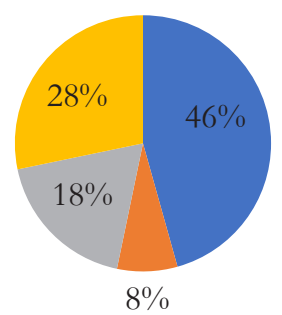

Figure 8. Participants who used a shopping list when buying groceries. 
Finally, the participants were asked if they used a shopping list when purchasing food items: 28\% indicated, "Often," $18 \%$ indicated, "Sometimes," and 46\% and 8\% responded "Never," and "Seldom," respectively, as Figure 8 shows. The participants who used shopping lists and added fresh fruits and vegetables to their lists demonstrated more intentional action in buying and consuming fresh produce.

\section{Qualitative Data: Emerging Themes}

Weekly research discussions highlighted themes that emerged from conversations with the participants. These were consistent across each of the wards, particularly the conversations with those who consumed lunch at congregant sites. While this population was guaranteed one balanced meal per day at the sites, it became apparent that its members might not eat other meals at all. Meals prepared and eaten at home often comprised frozen dinners that lacked adequate nutrition. Furthermore, the participants had varied views regarding what healthy and unhealthy food choices were Figure 9 and 10. There was surprising consistency among participants in the way they responded to whether the food selection was healthy versus unhealthy. Half of the participants in each group did not seem to find unhealthy food as being unhealthy. The themes were categorized as internal barriers, external barriers, and others as seen in Table 1. Many of the barriers that these participants identified were like those that emerged in many of the studies we reviewed in the literature.

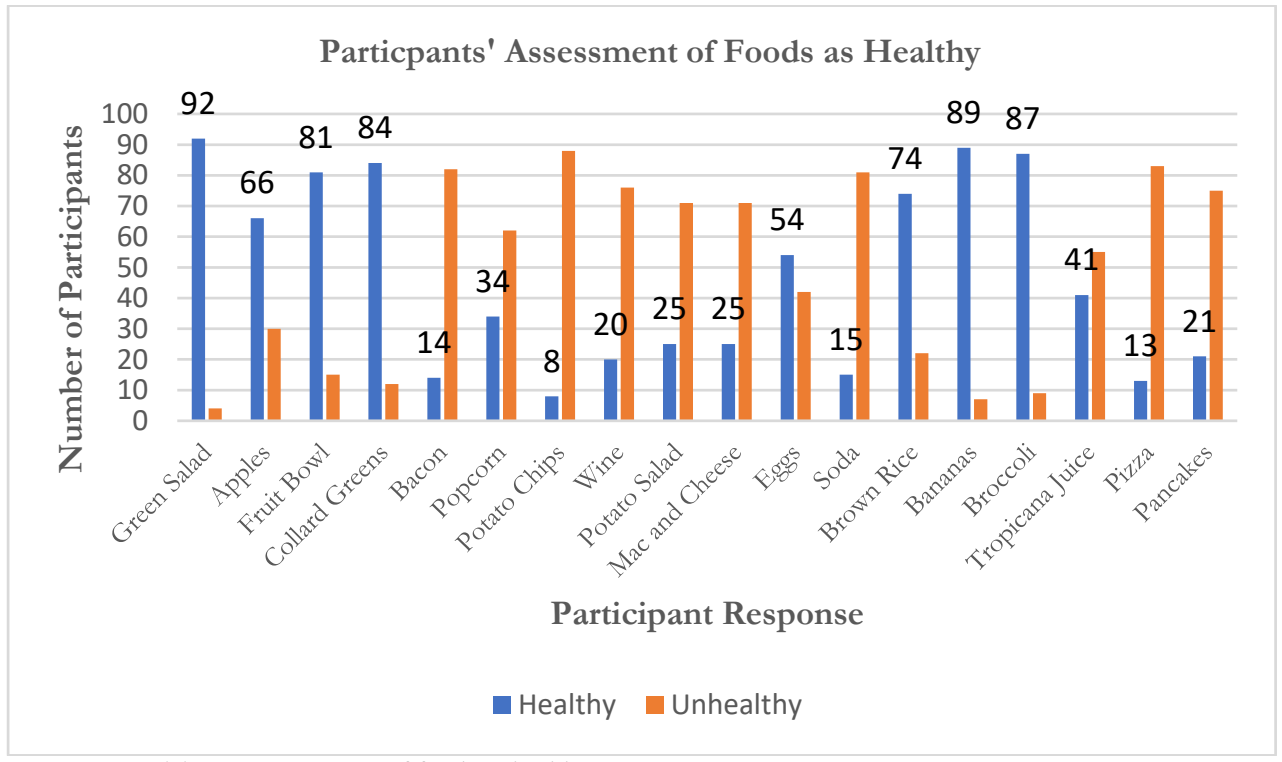

Figure 9. Participants' assessment of foods as healthy.

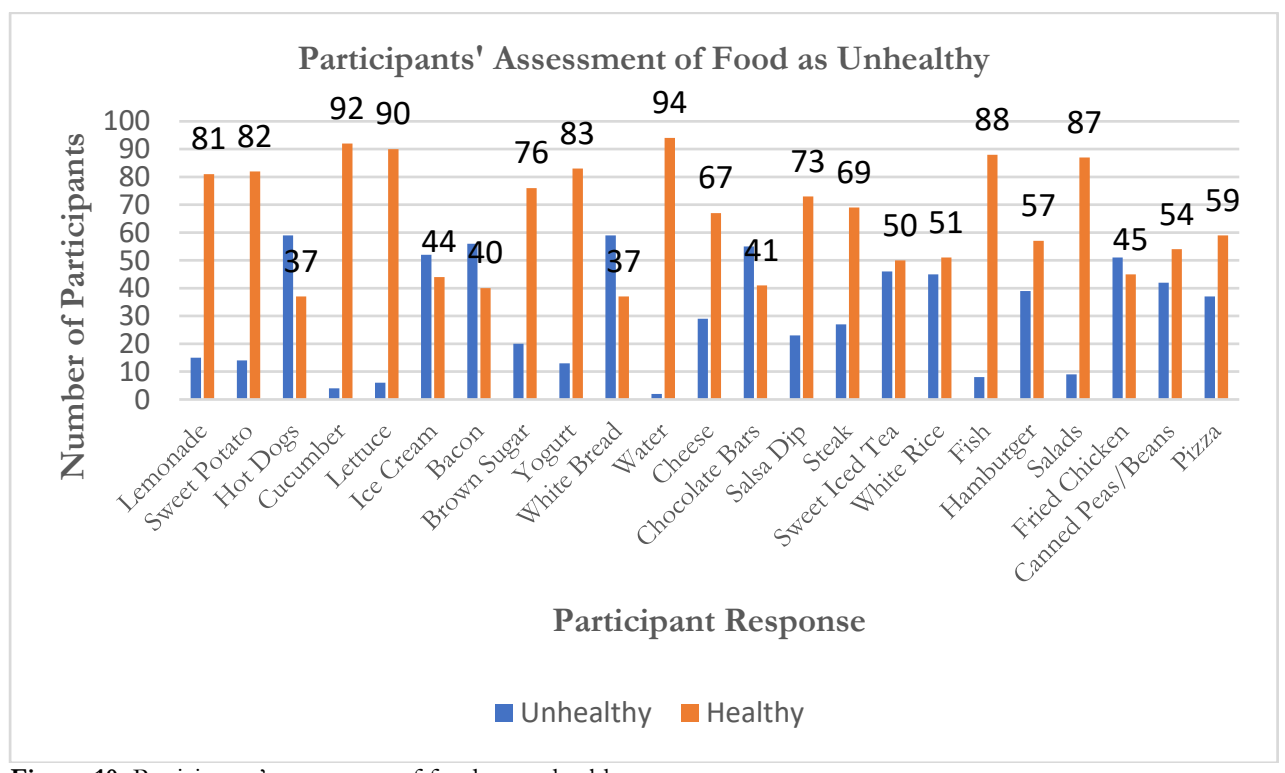

Figure 10. Participants' assessment of foods as unhealthy. 


\begin{tabular}{|c|c|c|}
\hline Barriers & Themes & Participants' Conversation \\
\hline Internal Barriers & $\begin{array}{l}\text { Meals not appealing } \\
\text { Institutional setting not appealing } \\
\text { Lack of nutritional knowledge on } \\
\text { the content of healthy vs. unhealthy } \\
\text { foods } \\
\text { Lack of knowledge regarding the } \\
\text { link between chronic conditions and } \\
\text { excess sodium and sugar intake } \\
\text { Cost - fixed income }\end{array}$ & $\begin{array}{l}\text { A Ward } 5 \text { participant who lived in a facility that was also a site } \\
\text { that provided congregate meals--for which they were } \\
\text { eligible-said, "Most chose not to receive those meals either } \\
\text { because they did not choose to socialize with other residents, } \\
\text { they did not feel that the meals were nutritionally sound, or } \\
\text { the meals did not appeal to them." }\end{array}$ \\
\hline External Barriers & $\begin{array}{l}\text { Not enough supermarkets in the } \\
\text { local neighborhood } \\
\text { Established grocers offer poor food } \\
\text { selection } \\
\text { Produce is of poor quality } \\
\text { Inadequate local transportation } \\
\text { system }\end{array}$ & $\begin{array}{l}\text { "I feel strongly the city government should do more to } \\
\text { provide more supermarkets closer to where you live." } \\
\text { "The further I go from the city, the better the quality of food" } \\
\text { Some seniors commented they would like to have a Whole } \\
\text { Foods store or Yes! Organic Market in their neighborhoods } \\
\text { When asked to explain, respondents cited transportation as } \\
\text { being an issue, as well as lack of choice and variety in grocery } \\
\text { stores } \\
\text { "I feel the traffic; closer stores are crowded" }\end{array}$ \\
\hline Other Barriers & $\begin{array}{l}\text { Other members of the community } \\
\text { could use help; those older than } 18 \\
\text { years but younger than } 60 \text { years. }\end{array}$ & $\begin{array}{l}\text { A respondent who was visually impaired stated that she "had } \\
\text { a friend in need of assistance, but there were no government } \\
\text { programs that provided help or relief like the services that her } \\
\text { friend receives." }\end{array}$ \\
\hline
\end{tabular}

Table 1. Barrier types.

\section{DISCUSSION}

The primary purpose of this project was to examine barriers that prevented residents of Wards 5,7 and 8 from accessing fresh fruits, vegetables, and whole grains. Fresh fruits, vegetables, and grains play a protective role, mitigating chronic diseases. Wards 5 , 7 , and 8 in the District of Columbia reported the highest prevalence of hypertension at 39.3\%, 41.5\%, and $40.4 \%$ respectively. ${ }^{27}$ The prevalence of diabetes was highest in Wards 5, 7 and 8, and death due to diabetes was highest in Ward 7. Diabetes mortality rate in Wards 5 (38.2\%), Ward 7 (44.6\%), and Ward $8(31.8 \%)$ as of 2008 and $2009 .{ }^{20}$ The research indicated that many of these chronic health conditions could be addressed with increasing African American compliance to the United States Department of Agriculture (USDA) recommendations for the daily consumption of fruits, vegetables, and whole grains. Sharma et al. stated that there was a need to reevaluate the dietary guidelines and that higher recommendation of fruits and vegetables might be necessary to reduce the risk of chronic diseases in high-risk populations. ${ }^{2}$ In 2015, the USDA released the 2015-2020 Dietary Guidelines.

Interestingly, the results show a surprising number of participants who stated they had access to fresh fruits, vegetables, and whole grains. This suggests that access was less of a barrier for $35 \%$ of these residents given the grocery store distribution in their neighborhoods. It is possible that this convenience sample of participants was in neighborhoods undergoing gentrification and was likely to have seen significant population shifts and an economic boom. There is evidence of economic growth in Ward 5, where several full-service grocery stores have entered the community, offering more fresh food options. Compare this to Ward 7 and Ward 8: There remain two full-service grocery stores in Ward 7 and only one full-service grocery store in Ward 8.

DC Hunger Solution reported data on the number of full-service grocery stores within the eight wards in Washington, DC and compared it to the median income level for each ward as seen in Table 2 . When the data on household income and the number of people in each of these wards was evaluated it was apparent there is a disparity in the number of grocery stores compared to the income level. ${ }^{28}$ The District of Columbia recognized that the grocery store gap is linked to the issue of food security. To combat this problem, the DC government passed the Food, Environment, and Economic Development Act (FEED-DC) and the Supermarket Tax Exemption Act of 2000 to attract grocery stores to low-income areas in the District. While these regulatory efforts were designed to provide incentives for grocery stores to locate in these areas, these incentives have not been able to attract large grocery chains. ${ }^{28}$ 


\begin{tabular}{|ccc|}
\hline Wards & \# of Full Service Grocery Stores & Median Income \\
\hline $\mathbf{1}$ & 8 & $\$ 80,794$ \\
\hline $\mathbf{2}$ & 7 & $\$ 99,422$ \\
\hline $\mathbf{3}$ & 9 & $\$ 109,909$ \\
\hline $\mathbf{4}$ & 5 & $\$ 71,545$ \\
\hline $\mathbf{5}$ & 7 & $\$ 55,063$ \\
\hline $\mathbf{6}$ & 10 & $\$ 90,903$ \\
\hline $\mathbf{7}$ & 2 & $\$ 39,828$ \\
\hline $\mathbf{8}$ & 1 & $\$ 31,642$ \\
\hline
\end{tabular}

Table 2. Full-service grocery stores in each ward.

Perhaps this was the reason why full-service grocers did not consider Wards 7 and 8 to be a viable market to place grocery stores. Household income appears to be one of the driving factors motivating vendors to open full-service grocery stores in areas of the District of Columbia Figure 11. In addition, Wards 7 and 8 have an increased number of residents who receive some type of public assistance, as seen in Figure 12. In the District of Columbia, it is difficult to conclude a direct correlation between the number of grocery stores and the level of household income; what is clear is the inadequate number of full-service grocery stores in these wards. Moreover, there have been giant strides in efforts to bring more farmers markets to Wards 7 and Ward 8 . Perhaps the positive response to the question of access to fruits, vegetables, and whole grains suggests that farmers markets have stepped in to fill the gap left by full-service grocers. However, it is worth noting that this survey study did not collect consumption data. Thus, it is uncertain what effect access has on overall food purchase selections. Research regarding actual consumption versus the items the respondents claimed they consumed would be a good point of departure in the future.

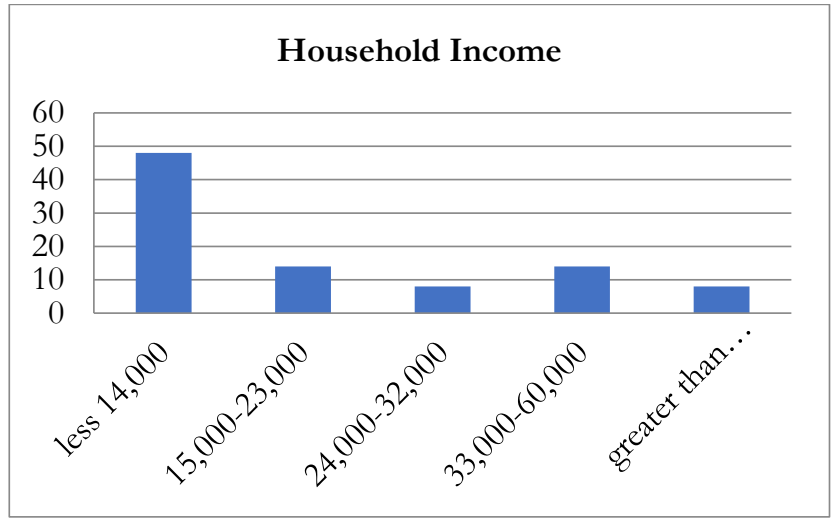

Figure 11. Household income.

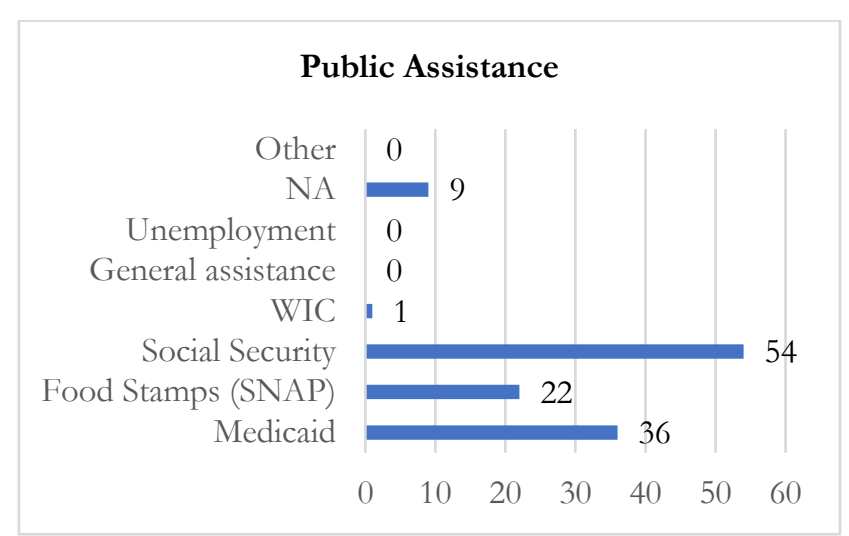

Figure 12. Participants receiving public assistance.

\section{CONCLUSIONS}

The findings from this research project answers our question: What are the barriers that prevent the consumption of fresh fruits and vegetables by older adults in $W$ ards 5, 7, and 8 in the District of Columbia? While cost, access to local quality fresh produce, transportation, are undeniably stated barriers; lack of nutritional knowledge and the selection of foods considered unhealthy was a surprising finding. The present research study provided some interesting insights into existing barriers that prevented access to fresh fruits and vegetables in Wards 5, 7, and 8. While these findings mirrored similar findings from the research literature, it was striking to confront some of these issues firsthand. While administering the surveys to the participants, the true meaning of food insecurity became evident. Food security as defined by the United States Department of Agriculture as "meaning, they lacked access to enough food for an active, health life for all household members" ${ }^{17}$ Fewer of the participants indicated that cost, transportation, or access was a barrier; however, it was revealed that most participants had differing views regarding foods they considered healthy. This became apparent when participants were asked to identify foods they considered healthy. The number of participants that chose unhealthy foods and marked them as healthy foods was startling. It is important to note that this project did not capture data on the length of time it took participants to travel to a full-service grocer; although, we believe, this data is relevant to further establish transportation as a barrier. 
For nutrition and dietetics research assistants and budding nutrition and dietetics professionals, opportunities to provide nutritional education to this targeted population abound; moreover, the situation presents an opportunity to improve the nutritional knowledge of all seniors across the District of Columbia. Currently, there are 48,809 persons over the age of 55. ${ }^{30}$ Health disparities continue to affect African Americans and people of color and as future nutrition and dietetic counselor one concrete strategy is to develop fact sheets aimed at increasing knowledge on diabetes and the linkage between an excess sugar and sodium intake. Another important educational strategy, particularly in each of these wards, is to develop posters that could be displayed at congregate meals sites that tell residents about the importance of eating the recommended servings of fruits and vegetables daily. The introduction of these two strategies perhaps will increase participants' knowledge of the importance of eating fruits and vegetables and will act as a catalyst to encourage a change in health behaviors.

These communities demonstrated a higher level of chronic disease such as diabetes than other communities in Washington, DC. Perhaps, one approach to address this would entail teaching residents how to prepare their traditional foods in a more healthful way. Finally, while the project did not discuss physical activity, encouraging physical activity among members of this target population must be part of the solution if the goal is to make Washington, DC, the greenest, healthiest city in the nation in accordance with the Sustainable DC Plan. ${ }^{7}$

\section{ACKNOWLEDGMENTS}

The authors thank Dr. Prema Ganganna, the survey participants and their respective site coordinators, the University of the District of Columbia Institute of Gerontology, Dr. Matthew Richardson, and Dr. Xiaochu Hu.

\section{REFERENCES}

1. Coleman-Jensen, A., Rabbitt, M.P., Gregory, C.A., Singh, A. (2017) Household Food Security in the United States in 2016. Economic Research Report No. (ERR-237) 44 pp. https://www.ers.usda.gov/publications/pub-details/?pubid=84972

2. Sharma, S., Pakserescht, M., Cruickshank, K., Green, D. M., Kolonel, L. N. (2013) Adherence to the USDA dietary recommendations for fruit and vegetable intake and risk of fatal stroke among ethnic groups: a prospective cohort study, $B M C$ Neurol, 13(120), 1-8. https:/ / doi.org/10.1186/1471-2377-13-120

3. DC Department of Health Cancer and Chronic Disease Prevention (n.d.) Diabetes in the District of Columbia Fact Sheet bttps:/ / dchealth.dc.gov/sites/default/files/dc/sites/doh/Diabetes\%20in\%20the\%District\%20of\%20Columbia\%20Fact\%20Sheet\%201105 2012.pdf https:// dchealth.dc.gov/ service/ cancer-and-chronic-disease-prevention (accessed Mar 2018)

4. United States Renal Data Systems 2011; Mid Atlantic Renal Coalition ESRD Network 5, 2010 Annual Report

5. Center for Disease Control and Prevention. Chronic Kidney Disease Surveillance System-United States. http:// wmw.cdc.gov/cked (accessed Mar 2018)

6. Sharma, S., Pakserescht, M., Cruickshank, K., Green, D. M., Kolonel, L. N. (2013) Adherence to the USDA dietary recommendations for fruit and vegetable intake, ethnicity and ischemic heart disease mortality, Nutr Metab Cardiovasc Dis, 23(12), 1247-1254. http:// dx.doi.org/10.1016/j.numecd.2013.03.004

7. Sustainable D.C. (2010) Sustainable DC Plan, https://sustainable.dc.gov/sites/default/files/dc/sites/sustainable/page_content/attachments/DCS-008\%20Report\%20508.3j.pdf(accessed Mar 2018)

8. Haynes-Maslow, L., Parsons, S. E., Wheeler, S. B., Leone, L. A. (2013) A qualitative study of perceived barriers to fruit and vegetable consumption among low-income populations, North Carolina, 2011. Preventing Chronic Disease 10, 1-10. https:/ / dx.doi.org/ 10.5888/pcd10.120206

9. Brewer, D., Dickens, E., Humphrey, A., Stephenson, T. (2016). Increased fruits and vegetable intake among older adults participating in Kentucky's congregate meal site program, Education Gerontology, 42(11), 771-784. Doi:10.1080/03601277.2016.1231511

10. Nicklett, E.J., Kadell, A.R. (2013). Fruit and vegetable intake among older adults: a scoping review. Maturitas 75(4),305-312. Doi: 10.1016/j.maturitas.2013.05.005

11. Morales, L. (2010) In U.S., consumption of fruits and vegetables trail access, Wellbeing, bttp:/ / news.gallup.com/poll/143159/consumption-fruits-vegetables-trails-access.aspx (accessed Mar 2018)

12. Resnicow, K., Jackson, A., Wang, T., De, A. K., McCarty, F., Dudley, W. N., Baranowski, T. (2001) A motivational interviewing intervention to increase fruit and vegetable intake through black churches: Results of the Eat Life trial, $A m J$ Public Health, 91 (10), 1686-1693.

13. Lucan, S. C., Barg, F. K., Long, J. A. (2010) Promoters and barriers to fruit, vegetable, and fast food consumption among urban, low-income African Americans_a qualitative approach, Am J Public Health, 100 (4), 631-635. Doi: 10.2105/AJPH.2009.172692

14. John, J. H., Ziebland, S. (2004) Reported barriers to eating more fruit and vegetables before and after participation in a randomized controlled trial: A qualitative study, Health Education Research Theory \& Practice, 19 (2), 165-174. Doi: 10.1093/ her/gyg016. 
15. Stephen, T., Troutman, A., Johnson, L., Taylor, T. (2015) Barriers to fresh fruit and vegetable intake among African Americans in a southeastern city: Preventive implication for cardiovascular disease. Journal of Family Medicine and Disease Prevention, 1:003.

16. Pinsker, E. A., Enzler, A. W., Hoffman, M. C., Call, K. T., Amos, S., Babington-Johnson, A., Okuyemi, K. S. (2017) A community-driven implementation of the body and soul program in churches in the twin cities, Minnesota 2011-2014, Prev Chronic Dis, 14 (E26), 1-11. http:/ / dx.doi.org/10.5888/pcd14.160386

17. Allicock, M., Johnson, L., Leone, L., Carr, C., Walsh, J., Ni, A., Resnicow, K., Pignone, M., Campbell, M. (2013). Promoting fruit and vegetable consumption among members of black churches, Michigan and North Carolina, 2008-2010. Preventing Chronic Disease Public Health Research, Practice, and Policy

18. Carson, J. S., Michalsky, L., Latson, B., Banks, K., Tong, L., Gimpel, N., Lee, J. J., DeHaven, M. J., (2012) The cardiovascular health of urban African Americans: Dietary results from the genes, nutrition, exercise, wellness, and spiritual growth (GoodNews) Trial, J Acad Nutr Diet, 112(11), 1852-1858. http:/ / dx.doi.org/10.1016/j.jand.2012.06.357

19. Satia, J. A., Galanko, J. A., Neuhouser, M. L. (2005) Food nutrition label use is associated with demographic, behavioral, and psychosocial factors and dietary intake among African Americans in North Carolina, J Am Diet Assoc, 105(3), 392-402. bttp:/ / dx.doi.org/10.1016/j.jada.2004.12.006

20. Lewis, J. E., Arheart, K. L., LeBlanc, W. G., Fleming, L. E., Lee, D. J., Davila, E. P., Caban-Martinez, A. J., Dietz, N. A. McCollister, K. E., Bandiera, F. C., Clark, J. D. (2009) Food label use and awareness of nutritional information and recommendations among persons with chronic disease, Am J Clin Nutr, 90, 1351-1357. bttps://10.3945/ajcn.2009.27684

21. Wright, R. S., Waldstein, S. R., Kuczmarki, M. F., Pohlig, R. T., Gerassimakis, C. S., Gaynor, B., Evans, M. K., Zonderman, A. B. (2016) Diet quality and cognitive function in an urban sample: Findings from the healthy aging in neighborhoods of diversity across the life span(HANDLS) study, Public Health Nutr, 20 (1), 92-101. https:// doi.org/ 10.1017/S136980016001361

22. Staub, B., Camus-Doinon, N., Bacon, E., Bonnefond, A. (2014) The effects of aging on sustained attention ability: An ERP Study, Psychol Aging, 29(3), 684-695. http:// dx.doi.org/10.1037/a0037067

23. Sen, S. (2017) Why Buddha never had Alsheimer's: A holistic treatment approach through meditation, Yoga, \& the arts $1^{\text {st }}$ ed., Health Communications, Inc., Deerfield Beach.

24. Kicklighter, J. R., Whitley, D. M., Kelley, S. J., Shipskie, S. M., Taube, J. L., Berry, R. C. (2007) Grandparents raising grandchildren: a response to a nutrition and physical activity intervention, J Am Diet Assoc, 107(7), $1210-1213$. bttp:/ / dx.doi.org/10.1016/j.jada.2007.04.006

25. Higgins, M. M., Murray, B. J. (2010) Nutrition-related practices and attitudes of Kansas skipped-generation(s) caregivers and their grandchildren, Nutrients, 2, 1188-1211. bttps:// doi.org/10.3390/ nu2121188

26. Treadwell, D. (2017) Introducing communication research paths of inquiry 3rd ed., 81, 139, Sage Publications, Los Angeles.

27. Schwartzman, P. (2017) D.C.'s grocery gap reflects city's income divide, The $W$ ashington Post, https: / / www.washingtonpost.com/ local/ dc-politics/dcs-grocery-gap-reflects-citys-income-divide/2017/06/01/874f0870-4627-11e7-bcde624ad94170ab_story.html?utm_term $=.92616 e d e 407 c$ (accessed Jan 2018)

28. DC Hunger Solution. (2017). Closing the grocery store gap in the nation's capital and race 2016 and 2017 , https:// suburbanstats.org/population/ how-many-people-live-in-washington-dc (accessed Dec 2017)

\section{ABOUT THE STUDENT AUTHORS}

Tornia Anderson-Morgan is a senior pursuing a BS in Nutrition and Dietetics and is an Undergraduate Research Assistant at The University of the District of Columbia (UDC). Her passion for wellness is reflected in her leadership roles at UDC and her volunteerism in the community. As President of the Student Association of Nutrition and Dietetics (SAND) and Executive Vice President of the National Society of Collegiate Scholars, she is actively involved in student development. Tornia also interns as a Fitness Instructor and Nutrition Educator with Montgomery County middle schools in Maryland. She aids the Health and Nutrition Officer at the Edward C. Mazique Child Care Center in Washington, DC, weekly and will pursue a career in nutrition and fitness. Melissa Fett is a senior pursuing a BS in Nutrition and Dietetics at UDC. She currently holds a BS in Public Policy and previously worked in the United States Senate as a legislative aide in health care policy. Upon her graduation from UDC in May and the completion of her Registered Dietitian certification exam, she plans to help close the food access and education gap in the DC metro area. She currently works as an Undergraduate Research Assistant on the NE-1439 Multi-State Research Project and has previous experience interning with DC Greens' Fruits and Vegetable Prescription Program and Arcadia Farm as a nutrition educator. She is also involved on the UDC campus as the Vice President of SAND. Michelle Jasso currently holds an Associate of Technology in Food Service Management and is pursuing a BS in Nutrition and Dietetics, which she will complete in Spring of 2018. She plans to work with the senior population in the DMV area when she completes her certification exam to become a Registered Dietitian. She currently works as an Undergraduate Research Assistant on the NE-1439 Multi-State Research Project: Changing the Health Trajectory for Older Adults through Effective Diet and Activity Modifications. Moreover, she previously worked as a student assistant for UDC CAUSES Ethnic Food Crop Program, which researched a variety of plants and farming techniques and helped residents in the DMV learn to grow their crops sustainably, manage them, and market them. 
Aisha Moten holds a BA in Fine Arts and graduated in the class of 2017 of the University of the District of Columbia, earning a BS in Nutrition and Dietetics. She currently works full time for the District of Columbia Department of Parks and Recreation and worked as an undergraduate research assistant at the University of the District of Columbia on the NE-1439 Multi-State Research Project. She plans to become a Registered Dietitian and to work in pediatrics and become a diabetes educator.

\section{PRESS SUMMARY}

The UDC-1439 project designed a pilot study to determine the existing barriers that prevented the consumption of fresh fruits, vegetables, and whole grains among older adults in Wards 5, 7, and 8 of the District of Columbia. These wards have higher incidences of diet-related chronic diseases, such as diabetes and hypertension. Survey participants included those aged 45 and older, with African Americans being the majority ethnic group represented. The 96 participants provided quantitative and qualitative data regarding barriers to wellness, including educational barriers, access, and socioeconomic factors. The data captured the surprising notion that education was a greater barrier than access. 


\title{
Minimizing the Cost of Guessing Games
}

\author{
David Clark ${ }^{a}$, Lindsay Czap ${ }^{* b}$ \\ ${ }^{a}$ Department of Mathematics, Grand Valley State University, Allendale, MI \\ ${ }^{b}$ Department of Mathematics, Middle Tennessee State University, Murfreesboro, TN
}

Students: czapl@mail.gvsu.edu,lnc4a@mtmail.mtsu.edu

Mentor: clarkdav@gvsu.edu

\begin{abstract}
A two-player "guessing game" is a game in which the first participant, the "Responder," picks a number from a certain range. Then, the second participant, the "Questioner," asks only yes-or-no questions in order to guess the number. In this paper, we study guessing games with lies and costs. In particular, the Responder is allowed to lie in one answer, and the Questioner is charged a cost based on the content of each question. Guessing games with lies are closely linked to error correcting codes, which are mathematical objects that allow us to detect an error in received information and correct these errors. We will give basic definitions in coding theory and show how error correcting codes allow us to still guess the correct number even if one lie is involved. We will additionally seek to minimize the total cost of our games. We will provide explicit constructions, for any cost function, for games with the minimum possible cost and an unlimited number of questions. We also find minimum cost games for games with a restricted number of questions and a constant cost function.
\end{abstract}

\section{KEYWORDS}

Ulam’s Game; Guessing Games With Lies; Error Correcting Codes; Pairwise Balanced Designs; Steiner Triple Systems

\section{INTRODUCTION}

The game "20 Questions" has always been a staple for children trapped in long car rides. This game involves two players: the first player picks an object and the second player asks "yes or no" questions in order to guess which object the first picked. The game alternates between questions and answers so questions may be constructed based off of previous answers. This type of adaptive guessing game is called an online game. For example, you would not ask the question "Does it have four legs?" if you have already received a "yes" to the question "Is your object a vegetable?"

"20 Questions" requires both players to be present and for each response to be given immediately after its corresponding question. In this paper, we will focus on a different type of guessing game: In an offline guessing game, the questioner must ask all of the questions at once and then receive all of the responses at once afterwards. This means that we must construct all of the questions ahead of time and we cannot adapt questions based upon previous responses. These types of non-adaptive guessing games are called offline games.

In our offline guessing games, the two players decide on a range of $m$ integers starting at 1 . The first player, the "Responder," secretly chooses one of those integers $x$. The second player, the "Questioner," then constructs yes-or-no questions of the form "Is $x \in S$ ?" where $S \subseteq\{1,2, \ldots, m\}$. Since these games are offline, all of the questions that the Questioner wishes to ask the Responder will be asked at once and then all of the answers will be received at once.

Example 1. The following is an example of an offline guessing game with $m=4$ possible answers.

\section{Questioner:}

Question 1: "Is your number in $\{3,4\}$ ?"

Question 2: "Is your number in $\{2,4\}$ ?"

Question 3: "Is your number in $\{2,3\}$ ?"

Question 4: "Is your number in $\{2,4\}$ ?"

Question 5: "Is your number in $\{3,4\}$ ?"

Responder: Responses: “Yes”, “No”, “Yes”, “No”, “Yes” (in order). 
The Questioner can then use the Responder's responses to figure out which integer was chosen. A response of “yes" to Question 1 eliminates the possibilities $\{1,2\}$, leaving only $\{3,4\}$ as possibilities. A response of "no" to Question 2 eliminates the possibility of 4 being the correct number, leaving only 3 as a possibility. Note that Question 2 also eliminates 2 as a possibility, but the Responder's answer to Question 1 already told us this. The remaining answers are no longer necessary, but they serve to confirm this result (and will be useful later, when we begin to discuss guessing games with lies). Thus, the Questioner knows that the Responder chose $x=3$ as their integer.

The guessing games that we will be analyzing have a special property: The Responder is allowed to lie to the Questioner. However, even though the Responder is allowed to lie, these games are designed so that the Questioner can still guess the correct number that the Responder has chosen. We will also add the idea of cost to a guessing game: The Questioner will be charged a certain cost whenever they ask a question. In the following sections, we will give precise definitions and background for each of these additional twists.

\section{BACKGROUND}

In this section, we give background and definitions, leading up to a precise definition of guessing games with lies and cost functions.

Guessing games with lies have been extensively studied. The idea of a "searching game" with a lie was suggested by Ulam in his autobiography ${ }^{1}$, which in turn spawned a large and active field of research. In general, work in this area has focused on minimizing the number of questions needed to determine the Responder's number correctly. In particular, Spencer ${ }^{2}$ focused on solving "Ulam's game" with 1,000,000 numbers and 1 lie, which turns out to be the equivalent of the "20 questions" game with 1 lie permitted. Offline guessing games with lies turn out to be equivalent to important objects in mathematics called error-correcting codes. See ${ }^{3,4}$ for some excellent surveys of the literature on guessing games with lies.

Our particular focus in this paper will be adding the idea of a "cost function" to offline guessing games with lies. A guessing game $G$ with cost function $c$ is an offline guessing game in which each of the possible answers $i$ is assigned a "cost" $c(i)$. For each question, the Questioner will be "charged" the cost of all of the answers that they are asking about. For example, the cost of the question "Is your number in $\{1,3\}$ ?" will be the sum of the costs of 1 and 3 . This generalizes the idea of minimizing the number of questions required, and allows us to measure the "efficiency" of a game in a more general way.

\section{Representations of guessing games}

For our offline guessing games, we will represent the answers to each of our questions using binary vectors. A binary vector is an ordered list of 1's and 0's $\left(x_{1}, x_{2}, \ldots, x_{n}\right)$ where $x_{i}=0$ if the Responder's answer to the $i$ th question is "no", and $x_{i}=1$ if the answer is yes. For example, if we asked 5 consecutive questions and their respective answers were "yes”, "yes","no," “yes”,"no”, we would represent these responses with the binary vector $(1,1,0,1,0)$. We will often use the shorthand notation: 11010.

We can think of an answer vector as the "fingerprint" of the Responder's secret number. It contains all of the information necessary to determine the secret number. In particular, in every guessing game, every secret number must have a different answer vector or else it would be impossible to distinguish between some of the numbers.

Definition 2. For a given natural number $m$, a cost function $c$ is a function $c:\{1,2, \ldots, m\} \rightarrow \mathbb{R}^{+}$with $0<c(1) \leq$ $c(2) \leq \ldots \leq c(m)$. For simplicity, we often represent $c$ as a vector: $c=(c(1), c(2), \ldots, c(m))$.

We can now define the fundamental object in this paper:

Definition 3. An ( $m, c, \ell)$ offline guessing game $G$ with $n$ questions is an ordered list of $n$ questions of the form "Is your number in the set $S_{i}$ ?", where $S_{i} \subseteq\{1,2, \ldots, m\}$, together with a cost function $c=(c(1), c(2), \ldots, c(m))$. The questions are arranged so that the Questioner can determine the Responder's secret number, even if the Responder is allowed to lie in at most $\ell$ answers. 
We will often omit the word "offline", since we will only be working with offline guessing games in this paper. Note that we have not yet indicated how the questions may be arranged to let the Questioner determine the Responder's secret number in the face of lies. We will address this in the following sections.

Next, we give a more precise definition of the "answer vectors" described at the beginning of this section. Here, let $\mathbb{F}_{2}^{n}$ be the space of all vectors of length $n$ over the finite field $\mathbb{F}_{2}$, that is, binary vectors of length $n$.

Definition 4. Given an $(m, c, \ell)$ guessing game $G$ with $n$ questions, the answer vector for $i$, denoted $\mathbf{v}_{i}$, is the binary vector $\mathbf{v}_{i} \in \mathbb{F}_{2}^{n}$ consisting of the Responder's truthful answers to the $n$ questions, in order, assuming that the Responder's secret number is $i$. The answer vector set of $G$, denoted $\mathcal{V}_{G}$, is the set $\left\{\mathbf{v}_{1}, \mathbf{v}_{2}, \ldots, \mathbf{v}_{m}\right\} \subseteq \mathbb{F}_{2}^{n}$ of answer vectors for $i=$ $1,2, \ldots, m$.

Definition 5. Given an $(m, c, \ell)$ game $G$ with $n$ questions, the guessing game matrix of $G$ is an $m \times n$ binary matrix denoted $[G]$ in which row $j$ corresponds to vector $\mathbf{v}_{j} \in \mathcal{V}_{G}$. That is, the $j$ th row of $[G]$ is the list of the Responder's truthful answers to the $n$ questions in order, assuming that the Responder's secret number is $j$.

Answer vectors and guessing game matrices are the main ways in which we will study guessing games in this paper. Because the rows of a guessing game matrix are the same as the answer vectors, we will often refer to the rows of a matrix as "vectors."

Example 6. There are many different vector sets that could be used to construct a $(4, c, 1)$ game. Consider the set $\mathcal{V}_{G}=$ $\{00000,01110,10101,11011\} \subseteq \mathbb{F}_{2}^{5}$. This is a set of 4 answer vectors of length 5 for the numbers $\{1,2,3,4\}$. This could be the answer vector set for a $(4, c, 1)$ guessing game with 5 questions. In particular, we have $\mathbf{v}_{1}=00000, \mathbf{v}_{2}=01110$, $\mathbf{v}_{3}=10101$, and $\mathbf{v}_{4}=11011$. Notice that $\mathbf{v}_{3}$ corresponds to the answers given by the Responder in Example 1.

Figure 1 shows the game matrix $[M]$ for a $(4, c, 1)$ game $M$ that uses the answer vector set $\mathcal{V}_{G}$.

$[M]=$\begin{tabular}{|c|ccccc|}
\hline & $q_{1}$ & $q_{2}$ & $q_{3}$ & $q_{4}$ & $q_{5}$ \\
\hline 1 & 0 & 0 & 0 & 0 & 0 \\
\hline 2 & 0 & 1 & 1 & 1 & 0 \\
\hline 3 & 1 & 0 & 1 & 0 & 1 \\
\hline 4 & 1 & 1 & 0 & 1 & 1 \\
\hline
\end{tabular}

Figure 1. A possible game matrix, $[M]$, for a $(4, c, 1)$ game.

It is possible to determine a game's questions entirely from its guessing game matrix. Consider the column labeled $q_{1}$ in Figure 1. We say that this column is the question vector $q_{1}=(0,0,1,1)$. We know that, if responding truthfully, the Responder will answer "yes" to this 1st question if and only if there is a 1 in the row of $[M]$ corresponding to their secret number. Therefore, $q_{1}$ would be the first question in the game $G$ and would be asked as: "Is your number in $\{3,4\}$ ?" because these are the answers whose answer vector have a 1 in column 1 of $[M]$. This is indeed the first question asked in Example 1.

We will often treat a guessing game not as a list of questions, but rather as a list of answer vectors. These two viewpoints are exactly equivalent.

Cost functions

We will now focus on the effect of the cost function in a guessing game. The cost function will be our measure of the "efficiency" of a game.

Definition 7. Let $G$ be an $(m, c, \ell)$ guessing game. Suppose that $Q=\left\{p_{1}, p_{2}, \ldots, p_{k}\right\} \subseteq\{1,2, \ldots, m\}$ is a question in $G$. Then the cost of question $Q$ is defined to be the sum of the costs of the elements of $Q$, that is,

$$
c(Q)=\sum_{i=1}^{k} c\left(p_{i}\right) .
$$

Equation 1. 
Furthermore, the total cost of $G$, denoted $k_{G}$, is the sum of the cost of $Q$ for all questions $Q$ in $G$, that is,

$$
k_{G}=\sum_{\text {question } Q} c(Q) .
$$

Equation 2.

Because our guessing games are offline, all of the questions will always be asked. Thus the total cost of a game is a reasonable measure of the overall "efficiency" of a game. The following gives us a convenient way to calculate the total cost of a game in terms of its answer vectors.

Definition 8. The weight of a vector $\mathbf{v} \in \mathbb{F}_{2}^{n}$, denoted $|\mathbf{v}|$, is the number of nonzero coordinates in $\mathbf{v}$.

Definition 9. The total cost $k_{G}$ of an $(m, c, \ell)$ guessing game $G$ with answer vector set $\left\{\mathbf{v}_{1}, \mathbf{v}_{2}, \ldots, \mathbf{v}_{m}\right\}$ is $k_{G}=c(1)\left|\mathbf{v}_{1}\right|+$ $c(2)\left|\mathbf{v}_{2}\right|+\ldots+c(m)\left|\mathbf{v}_{m}\right|$.

Example 10. We consider a $(4, c, 1)$ game $G$ with $c=(2,4,6,8)$ and game matrix $[M]$ as shown in Figure 1.

Reading the columns of matrix $[M]$, we see that the questions and their costs are:

\begin{tabular}{cll}
$\#$ & Question & Cost \\
$q_{1}$ & Is your number in $\{3,4\} ?$ & $6+8=14$ \\
$q_{2}$ & Is your number in $\{2,4\} ?$ & $4+8=12$ \\
$q_{3}$ & Is your number in $\{2,3\} ?$ & $4+6=10$ \\
$q_{4}$ & Is your number in $\{2,4\} ?$ & $4+8=12$ \\
$q_{5}$ & Is your number in $\{3,4\} ?$ & $6+8=14$ \\
\hline & Total: & 62
\end{tabular}

Using Definition 9, the total cost of $G$ is calculated by:

$$
\begin{aligned}
k_{G} & =\left|\mathbf{v}_{1}\right| c(1)+\left|\mathbf{v}_{2}\right| c(2)+\left|\mathbf{v}_{3}\right| c(3)+\left|\mathbf{v}_{4}\right| c(4) \\
& =0 c(1)+3 c(2)+3 c(3)+4 c(4) \\
& =0 \cdot 2+3 \cdot 4+3 \cdot 6+4 \cdot 8 \\
& =62 .
\end{aligned}
$$

This demonstrates the essence of Definition 9: It is possible to count the costs of a game by using the columns of its guessing game matrix (questions), or by using its rows (answer vectors).

\section{Error-correcting codes and lies}

In order to discuss the ability for our $(m, c, \ell)$ guessing games to detect and correct for lying, we must first introduce some ideas from a branch of mathematics known as coding theory. We refer the interested reader to Huffman and Pless ${ }^{5}$ for further definitions and examples.

Definition 11. The Hamming Distance between two vectors $\mathbf{x}, \mathbf{y} \in \mathbb{F}_{2}^{n}$, denoted $d(\mathbf{x}, \mathbf{y})$, is the number of coordinates in which $\mathbf{x}$ and $\mathbf{y}$ differ.

Definition 12. The minimum distance $d(C)$ of a set of vectors $C \subseteq \mathbb{F}_{2}^{n}$ is the smallest Hamming Distance between any two distinct vectors in $C$.

Example 13. Continuing to use the answer vectors from Example 6, integers 2 and 3 have answer vectors $\mathbf{v}_{2}=01110$ and $\mathbf{v}_{3}=10101$. These vectors differ in 4 positions - every position except the middle digit, where both have 1's. Thus $d\left(\mathbf{v}_{2}, \mathbf{v}_{3}\right)=4$.

The smallest distance among any pair of vectors in $\mathcal{V}_{G}$ from Example 6 is 3 (between $\mathbf{v}_{1}$ and $\mathbf{v}_{2}$, as well as others), and so $d\left(\mathcal{V}_{G}\right)=3$.

With these ideas in hand, we are ready to define the fundamental idea that will allow us to detect and correct lies in guessing games. 
Definition 14. An $(n, M, d)$ error-correcting code $C$ (or "ECC") is a subset of $\mathbb{F}_{2}^{n}$ of size $M$ with $d(C)=d$. We call these vectors in $C$ codewords. An $(n, M, d)$ error-correcting code can be represented by an $M \times n$ matrix denoted $[C]$ whose rows are the codewords of $C$.

An ECC has the capacity to detect and then correct for errors that can occur in a codeword when it is exposed to contextual factors. These factors can include noise over a digital channel, a misprint in written text, or - in the case of our guessing games - a player lying. The capacity for an ECC to detect and correct for errors is based on the minimum distance of that code.

The process by which minimum distance allows a code to correct errors is called nearest-neighbor decoding. For an $(n, M, d)$ code $C$, a message vector $\mathbf{m}$ is any one codeword. It is transmitted through a channel, where errors occur in the form of bits "flipped" from 1 to 0 or vice versa. The received message vector $\mathbf{r}$ is then a binary vector with $n$ coordinates that may share some entries with $\mathbf{m}$. To decode this message, vector $\mathbf{r}$ is compared to all of the codewords in the code. The codeword $\mathbf{c}$ that minimizes $d(\mathbf{c}, \mathbf{r})$ is chosen as the "nearest neighbor" to $\mathbf{r}$. In particular, if there are at most $\ell$ mistakes in $\mathbf{r}$, and the minimum distance of a code is at least $2 \ell+1$, then there must be a unique codeword that is "closest" to $\mathbf{r}$ and which results in a correct decoding.

Proposition 15 (See Huffman and Pless $\left.{ }^{5}\right)$. An $(n, M, d)$ error-correcting code $C$ with $d \geq 2 \ell+1$ can detect and correct up to $\ell$ errors.

Example 16. We continue to work with the matrix $[M]$ shown in Figure 1 of Example 6. We interpret the rows of $[M]$ as the vectors in an error-correcting code $C$. It follows that $d(C)=3$ since the Hamming Distance between any two codewords in $C$ is at least 3 . Thus, $C$ can detect and correct for up to $\ell=1$ errors.

For example, if the vector $\mathbf{r}=(0,1,0,1,1)$ were received, we could compare its distance to each of the four vectors in $C$. The 4th vector has a distance of 1 to $\mathbf{r}$, and no other vector has a distance that is 1 or lower, and so $\mathbf{r}$ would be corrected as $(1,1,0,1,1)$.

A valid guessing game matrix $[G]$ for an $(m, c, \ell)$ game $G$ with $n$ questions can be viewed as the matrix for an $(n, m, 2 \ell+$ 1 ) error-correcting code and vice versa. The binary answer vectors in $[G]$ that are assigned to each of the $m$ possible integers correspond to the codewords of the code represented by $[G]$. The number of lies $\ell$ that are allowed in $G$ corresponds to the number of errors that the corresponding ECC can detect and correct. By this, it follows that in order for $[G]$ to be a valid guessing game matrix for an $(m, c, \ell)$ game $G$, it must be true that $\mathrm{d}([G]) \geq 2 \ell+1$. Thus, trying to find an $(m, c, \ell)$ offline guessing game $G$ with $n$ questions is equivalent to finding an $(n, m, 2 \ell+1)$ error-correcting code.

In the remainder of this paper, we will find $(m, c, 1)$ games with $n$ questions by solving the equivalent problem of finding $(n, m, 3)$ error-correcting codes. However, we will have the additional requirement of minimizing the total cost of the corresponding game, a restriction which is not normally considered when constructing an error-correcting code.

\section{RESULTS}

Guessing Games with an Unrestricted Number of Questions

In this section, we will make several assumptions. First, we restrict our investigations to guessing games allowing for only one lie, that is, $\ell=1$. Second, we place no restriction on the number of questions that the Questioner asks. Thus, for each guessing game we will specify only the number of possible answers $m$, and will leave the number of questions $n$ unrestricted. Instead, we will rely on the cost function for our games to measure the efficiency of the game.

For any $(m, c, 1)$ guessing game $G$, either the all-zeroes vector $\mathbf{0}$ is in the set of answer vectors $\mathcal{V}_{G}$, or it isn't. We will show two guessing game matrix constructions that we can use to minimize the total cost of a guessing game, depending upon the inclusion or exclusion of $\mathbf{0}$.

Definition 17. An $m \times n(3,0)$ matrix $[G]_{m}^{(3,0)}$ is an $m \times(2 m-1)$ binary matrix with the form shown in Figure 2.

That is, there are $m-1$ rows of weight 3 and a single row of weight 0 at the bottom. The rows of weight 3 are arranged in a "staircase" fashion so that each row shares a 1 in exactly one coordinate with the previous row, and in exactly one coordinate with the subsequent row. 


\begin{tabular}{|c|ccccccccc|}
\hline 1 & 1 & 1 & 1 & 0 & 0 & 0 & 0 & $\ldots$ & 0 \\
\hline 2 & 0 & 0 & 1 & 1 & 1 & 0 & 0 & $\ldots$ & 0 \\
\hline$\vdots$ & $\vdots$ & $\vdots$ & $\vdots$ & $\vdots$ & $\vdots$ & $\vdots$ & $\vdots$ & $\vdots$ & $\vdots$ \\
\hline$m-1$ & 0 & 0 & 0 & 0 & $\ldots$ & 0 & 1 & 1 & 1 \\
\hline$m$ & 0 & 0 & 0 & 0 & 0 & 0 & 0 & 0 & 0 \\
\hline
\end{tabular}

Figure 2. An $m \times n(3,0)$ matrix $[G]_{m}^{(3,0)}$.

Definition 18. An $m \times n(2,1)$ matrix $[G]_{m}^{(2,1)}$ is an $m \times(2 m-1)$ binary matrix with the structure shown in Figure 3 .

\begin{tabular}{|c|ccccccccc|}
\hline 1 & 1 & 1 & 0 & 0 & 0 & 0 & 0 & $\ldots$ & 0 \\
\hline 2 & 0 & 0 & 1 & 1 & 0 & 0 & 0 & $\ldots$ & 0 \\
\hline$\vdots$ & $\vdots$ & $\vdots$ & $\vdots$ & $\vdots$ & $\vdots$ & $\vdots$ & $\vdots$ & $\vdots$ & $\vdots$ \\
\hline$m-1$ & 0 & 0 & 0 & 0 & $\ldots$ & 0 & 1 & 1 & 0 \\
\hline$m$ & 0 & 0 & 0 & 0 & 0 & 0 & 0 & 0 & 1 \\
\hline
\end{tabular}

Figure 3. An $m \times n(2,1)$ matrix $[G]_{m}^{(2,1)}$.

There are $m-1$ rows of weight 2 and a single row of weight 1 at the bottom. No pair of rows share nonzero coordinates.

Note that, by construction, the rows of both $(3,0)$ and $(2,1)$ matrices have a minimum distance of 3 .

Lemma 19. Let $m$ and $c=(c(1), c(2), \ldots, c(m))$ be given. Among all $(m, c, 1)$ guessing games that contain an answer vector consisting of all $O$ 's, the minimum total cost is $k_{\mathbf{0} \in \mathcal{V}}=3 c(1)+3 c(2)+\ldots+3 c(m-1)$. A game with guessing game matrix $[G]_{m}^{(3,0)}$ will attain this minimum total cost.

Proof. Let $m$ and $c=(c(1), c(2), \ldots, c(m))$ be given. By Definition 9, the $(3,0)$ matrix $[G]_{m}^{(3,0)}$ has total cost $c\left([G]_{m}^{(3,0)}\right)=$ $3 c(1)+3 c(2)+\cdots+3 c(m-1)$. Next we will show that this is the minimum cost.

Let $[G]$ be any guessing game matrix for a $(m, c, 1)$ game with minimum total cost among all $(m, c, 1)$ games that contain a zero answer vector. By assumption, $[G]$ must contain a vector of weight 0 . Because $d([G]) \geq 3$, all other rows of $[G]$ must have weight at least 3. By Definition 9 and the fact that all costs are assumed to be positive, all other rows of $[G]$ must have weight exactly 3 .

Next we claim that the row of $[G]$ corresponding to answer $m$ must be $\mathbf{0}$. (Recall that, by assumption, $c(m)$ is the largest cost.) To prove this, we let $\left\{\mathbf{v}_{1}, \mathbf{v}_{2}, \ldots, \mathbf{v}_{i-1}, \mathbf{0}, \mathbf{v}_{i+1}, \ldots, \mathbf{v}_{m}\right\}$ be a fixed set of vectors with $\left|\mathbf{v}_{j}\right|=3$ for all $j$, minimum distance at least 3 , and $\mathbf{0}$ in position corresponding to cost $c(i), i \neq m$. It then follows that the cost of this game is

$$
k_{i}=3 c(1)+3 c(2)+\cdots+3 c(i-1)+0 c(i)+3 c(i+1)+\cdots+3 c(m-1)+3 c(m) .
$$

Suppose now that the same vectors are rearranged to place $\mathbf{0}$ in the $m$ th position. The cost of this new game is

$$
k_{m}=3 c(1)+3 c(2)+\cdots+3 c(i)+\cdots+3 c(m-1)+0 c(m) .
$$

Thus,

$$
k_{i}-k_{m}=3 c(m)-3 c(i) .
$$

Since $c(i) \leq c(m)$ by assumption, it then follows that $k_{i}-k_{m} \geq 0$ and therefore $k_{i} \geq k_{m}$. Thus it must be that $[G]$ contains a single vector of weight zero that represents the integer $m$, and all other vectors have weight 3 .

Therefore by Definition 9, this minimum cost must be

$$
c([G])=3 c(1)+3 c(2)+\ldots+3 c(m-1)=c\left([G]_{m}^{(3,0)}\right),
$$

and so a $(3,0)$ matrix $[G]_{m}^{(3,0)}$ attains the minimum cost. 
Lemma 20. Let $m$ and $c=(c(1), c(2), \ldots, c(m))$ be given. Among all $(m, c, 1)$ guessing games that do not contain an answer vector consisting of all $O$ 's, the minimum total cost is $k_{\mathbf{0} \notin \mathcal{V}}=2 c(1)+2 c(2)+\ldots+2 c(m-1)+c(m)$. A game with guessing game matrix $[G]_{m}^{(2,1)}$ will attain this minimum total cost.

Proof. Let $m$ and $c=(c(1), c(2), \ldots, c(m))$ be given. By Definition 9, the $(2,1)$ matrix $[G]_{m}^{(2,1)}$ has total cost $c\left([G]_{m}^{(2,1)}\right)=$ $2 c(1)+2 c(2)+\cdots+2 c(m-1)+c(m)$. Next we will show that this is the minimum cost.

Let $[G]$ be any guessing game matrix for a $(m, c, 1)$ game with minimum total cost among all $(m, c, 1)$ games that do not contain an answer vector of weight zero. We first claim that $[G]$ will contain a vector of weight 1 . Suppose such a vector does not exist in $[G]$. It would follow that every vector in $[G]$ has a weight of at least 2 . We will demonstrate that some vector may be reduced to a weight of 1 . Suppose the set of vectors $\left\{\mathbf{v}_{1}, \ldots, \mathbf{v}_{i}, \ldots, \mathbf{v}_{m}\right\}$ in Figure 4 a represent the rows of $[G]$. These rows satisfy $\mathrm{d}([G]) \geq 3$ and all have weight of at least 2 . Replace $\mathbf{v}_{i}$ with $\mathbf{0}$ and ask an additional question about only $i$, as illustrated in Figure $\mathbf{4 b}$. We can denote this new answer vector by $\mathbf{0} \mid 1$. It follows that $d\left(\mathbf{0}, \mathbf{v}_{j}\right) \geq 2$ for all $\mathbf{v}_{j}$, and because the single additional 1 occurs in a column of all 0 's, $d\left(\mathbf{0} \mid 1, \mathbf{v}_{j}\right) \geq 3$. Thus the original $\mathbf{v}_{i}$ can be changed to $\mathbf{0} \mid 1$ while maintaining $\mathrm{d}([G]) \geq 3$. This new matrix in Figure $4 \mathrm{~b}$ has a strictly lower total weight than the matrix in Figure $4 a$.

\begin{tabular}{|ccccccccc|}
\hline \multicolumn{1}{|c}{} & $\mathbf{v}_{1}$ & & & & \\
\hline$\vdots$ & $\vdots$ & $\vdots$ & $\vdots$ & $\vdots$ & $\vdots$ & $\vdots$ & $\vdots$ & $\vdots$ \\
\hline & \multicolumn{1}{|c}{} & $\mathbf{v}_{i}$ \\
\hline$\vdots$ & $\vdots$ & $\vdots$ & $\vdots$ & $\vdots$ & & & & \\
\hline \multicolumn{1}{|c|}{} & $\mathbf{v}_{m}$ & & & & \\
\hline
\end{tabular}

(a) The rows of $[G]$ that satisfy $\mathrm{d}([G]) \geq 3$.

\begin{tabular}{|cccccccccc|c|}
\hline & \multicolumn{1}{|c}{} & $\mathbf{v}_{1}$ & & & & & 0 \\
\hline$\vdots$ & $\vdots$ & $\vdots$ & $\vdots$ & $\vdots$ & $\vdots$ & $\vdots$ & $\vdots$ & $\vdots$ & \\
\hline 0 & 0 & 0 & 0 & 0 & 0 & 0 & 0 & 0 & 1 \\
\hline$\vdots$ & $\vdots$ & $\vdots$ & $\vdots$ & $\vdots$ & $\vdots$ & $\vdots$ & $\vdots$ & $\vdots$ & \\
\hline & & & $\mathbf{v}_{m}$ & & & & & 0 \\
\hline
\end{tabular}

(b) The rows of $[G]$ with $\mathbf{0}$.

Figure 4. Replacing vector $v_{i}$ in $[G]$ with $\mathbf{0}$.

If two vectors of weight 1 existed, then their Hamming Distance would be at most 2 and thus $\mathrm{d}([G]) \leq 2$. Thus $[G]$ contains exactly one vector of weight 1 . To ensure that $d([G]) \geq 3$, it follows that all other vectors must have a weight of at least 2. By Definition 9 and the fact that all costs are assumed to be positive, all $m-1$ rows of weight at least 2 have weight exactly 2. Thus $[G]$ will contain exactly one vector of weight 1 and $m-1$ vectors of weight 2 .

Therefore by Definition 9, this minimum cost must be

$$
c([G])=2 c(1)+2 c(2)+\cdots+2 c(m-1)+c(m)=c\left([G]_{m}^{(2,1)}\right)
$$

and so a $(2,1)$ matrix $[G]_{m}^{(2,1)}$ attains the minimum cost.

We are now ready to describe the minimum-cost guessing games, where the number of questions is unrestricted.

Theorem 21. Let $m$ and $c=(c(1), c(2), \ldots, c(m))$ be given.

- If $c(m)<\sum_{i=1}^{m-1} c(i)$ then the minimum cost of a $(m, c, 1)$ guessing game is realized by a $(2,1)$ matrix $[G]_{m}^{(2,1)}$.

- If $c(m)>\sum_{i=1}^{m-1} c(i)$ then the minimum cost of a $(m, c, 1)$ guessing game is realized by a $(3,0)$ matrix $[G]_{m}^{(3,0)}$.

- If $c(m)=\sum_{i=1}^{m-1} c(i)$ then the costs of a game realized by a $(3,0)$ matrix and $a(2,1)$ matrix are both equal to the minimum cost.

Proof. Let $m$ and $c=(c(1), c(2), \ldots, c(m))$ be given. Let $k_{(3,0)}$ and $k_{(2,1)}$ be the total cost of an $(m, c, 1)$ guessing game realized by a $(3,0)$ matrix and a $(2,1)$ matrix respectively. 


$\left[G_{1}\right]=$\begin{tabular}{|c|ccccccc|}
\hline & $q_{1}$ & $q_{2}$ & $q_{3}$ & $q_{4}$ & $q_{5}$ & $q_{6}$ & $q_{7}$ \\
\hline 1 & 1 & 1 & 1 & 0 & 0 & 0 & 0 \\
\hline 2 & 0 & 0 & 1 & 1 & 1 & 0 & 0 \\
\hline 3 & 0 & 0 & 0 & 0 & 1 & 1 & 1 \\
\hline 4 & 0 & 0 & 0 & 0 & 0 & 0 & 0 \\
\hline
\end{tabular}

$\left[G_{2}\right]=$\begin{tabular}{|c|ccccccc|}
\hline & $q_{1}$ & $q_{2}$ & $q_{3}$ & $q_{4}$ & $q_{5}$ & $q_{6}$ & $q_{7}$ \\
\hline 1 & 1 & 1 & 0 & 0 & 0 & 0 & 0 \\
\hline 2 & 0 & 0 & 1 & 1 & 0 & 0 & 0 \\
\hline 3 & 0 & 0 & 0 & 0 & 1 & 1 & 0 \\
\hline 4 & 0 & 0 & 0 & 0 & 0 & 0 & 1 \\
\hline
\end{tabular}

Figure 5. Two possible matrices for a $(4, c, 1)$ game.

By Lemmas 19 and 20, either a $(3,0)$ or a $(2,1)$ matrix gives the minimum total cost of an $(m, c, 1)$ game, depending on whether or not a zero vector is present. Thus one of these two must represent the minimum possible cost of an $(m, c, 1)$ game.

Consider the case where $c(m)<\sum_{i=1}^{m-1} c(i)$. Using our assumptions and calculating the total cost of a $(2,1)$ and $(3,0)$ matrix, we have:

$$
\begin{aligned}
k_{(2,1)} & =2 c(1)+2 c(2)+\cdots+2 c(m-1)+c(m) \\
& <2 c(1)+2 c(2)+\cdots 2 c(m-1)+\sum_{i=1}^{m-1} c(i) \\
& =3 c(1)+3 c(2)+\ldots+3 c(m-1)+0 c(m) \\
& =k_{(3,0)} .
\end{aligned}
$$

Thus, $c(m)<\sum_{i=1}^{m-1} c(i)$ implies $k_{(2,1)}<k_{(3,0)}$. Therefore, a $(2,1)$ matrix gives the cheapest possible total cost. Similarly, $c(m)>\sum_{i=1}^{m-1} c(i)$ implies $k_{(2,1)}>k_{(3,0)}$, is which case a $(3,0)$ matrix gives the cheapest possible total cost. If $c(m)=\sum_{i=1}^{m-1} c(i)$ then these two matrices give equal total costs.

Example 22. Let $m=4$. The two candidates for the cheapest $(4, c, 1)$ guessing game are given in Figure 5.

If $c=(1,2,3,4)$, then $c(4)=4<1+2+3=\sum_{i=1}^{3} c(i)$. Theorem 21 predicts that a $(2,1)$ matrix will be cheapest. We calculate: $c\left(\left[G_{1}\right]\right)=3(1)+3(2)+3(3)=18$ and $c\left(\left[G_{2}\right]\right)=2(1)+2(2)+2(3)+1(4)=16$. Thus the $(2,1)$ matrix does indeed realize the cheapest game.

If instead $c=(1,2,3,10)$, then $c(4)=10>1+2+3=\sum_{i=1}^{3} c(i)$. Theorem 21 predicts that a $(3,0)$ matrix will be cheapest. We calculate: $\left(\left[G_{1}\right]\right)=3(1)+3(2)+3(3)=18$ and $\left(\left[G_{2}\right]\right)=2(1)+2(2)+2(3)+1(10)=22$. Thus the $(3,0)$ matrix is cheaper in this case.

\section{Guessing Games with a Restricted Number of Questions}

In the previous section, we saw that the cheapest guessing games required us to create a very large number of questions. In particular, any guessing game with $m$ possible answers requires $2 m-1$ questions. We will now look at guessing games where the number of questions are restricted. As a simplification, we will only consider the cost function $c=(1,1, \ldots, 1)$. We begin, as we did in the previous section, by defining a special type of matrix that will be used throughout our study of this topic.

Definition 23. An $m \times n(1,2,3)$ matrix $[G]$ is a matrix with minimum distance 3 which has the form shown in Figure 6. Here, $[\mathrm{A}]$ is a matrix with rows of weight 3 , and $[\mathrm{B}]$ is a matrix with $\left\lfloor\frac{n-1}{2}\right\rfloor$ rows of weight 2 .

Note that, to guarantee a minimum distance of at least 3 , no row of weight 2 or 3 can have a 1 in the rightmost column. In addition, $\left\lfloor\frac{n-1}{2}\right\rfloor$ is the largest number of rows of weight 2 that can be contained in a matrix with $n-1$ columns and minimum distance at least 3 . No two rows with weight 2 can share a 1 in any position.

Next, we will show that $(1,2,3)$ matrices (when they exist) give the lowest possible cost. 


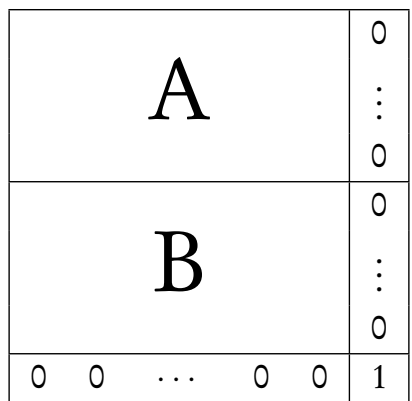

Figure 6. A $(1,2,3)$ matrix, [G].

Theorem 24. Let $c=(1,1, \ldots, 1)$ and let $n \geq 3$. If a $m \times n(1,2,3)$ matrix $[M]$ exists, then $[M]$ attains the minimum total cost for an $(m, c, 1)$ guessing game with exactly $n$ questions. This minimum cost is exactly $3\left(m-\left\lfloor\frac{n-1}{2}\right\rfloor-1\right)+2\left\lfloor\frac{n-1}{2}\right\rfloor+1=$ $3 m-\left\lfloor\frac{n-1}{2}\right\rfloor-2$.

Proof. Let $m$ and $n$ be parameters for an $(m, c, 1)$ guessing game with exactly $n$ questions, and assume an $m \times n(1,2,3)$ matrix $[G]$ exists. Because the cost function is fixed at $c=(1,1, \ldots, 1)$, the total cost of a $(m, c, 1)$ guessing game with game matrix $[G]$ is exactly the number of 1's in $[G]$. Thus our goal is to determine the minimum total number of 1's among all $m \times n$ binary matrices $[G]$ which have a minimum distance of at least 3 .

Let $[G]$ be a $m \times n$ guessing game matrix with $d([G]) \geq 3$ that attains the minimum possible number of 1 's.

We first claim that $[G]$ does not contain a zero vector. If $[G]$ did contain a zero vector, then to ensure $d([G]) \geq 3$, all other vectors must have weight at least 3 . Note that $[M]$ must contain at least one row of weight 2 , and so this guarantees that $[G]$ has at least as many 1's as $[M]$. Thus we may assume that $[G]$ does not contain a zero vector.

Next, we claim that $[G]$ must contain a single vector of weight 1 . Certainly $[G]$ cannot have two or more vectors of weight 1 while maintaining $d([G]) \geq 3$, and so it can contain either 1 or 0 such vectors. Suppose it has none. Then even if $[G]$ has the maximum possible number of rows of weight $2,[G]$ must have at least one more vector of weight 3 or larger compared to $[M]$. Thus $[G]$ would not have lower weight than $[M]$.

Thus we may assume that $[G]$ has no zero vector and has exactly one vector of weight 1 . Without loss of generality, assume that this single 1 appears in column $n$. To ensure $d([G]) \geq 3$, any rows of weight 2 may not have a 1 in column $n$. Thus at most $\left\lfloor\frac{n-1}{2}\right\rfloor$ of the remaining rows may have weight 2 , and the rest must have weight at least 3 . A $(1,2,3)$ matrix meets these bounds exactly, and so it must represent the minimum possible cost.

Note that the cost of an $m \times n(1,2,3)$ matrix is $3\left(m-\left\lfloor\frac{n-1}{2}\right\rfloor-1\right)+2\left\lfloor\frac{n-1}{2}\right\rfloor+1$, because $\left\lfloor\frac{n-1}{2}\right\rfloor$ of the $m$ rows have weight 2 , one has weight 1 , and the rest of the $m$ rows have weight 3 .

In the following work, we will prove that $(1,2,3)$ matrices exist in certain cases. To do so requires some concepts from a branch of math known as Design Theory. The interested reader is referred to Lindner ${ }^{6}$ for more information beyond that presented below.

Definition 25. Let $X=\{1,2, \ldots, u\}$ for $u \geq 3$ (these elements are called varieties), together with a set $B$ of subsets of $X$ of size 3 (called blocks or triples), such that every pair of varieties in $X$ occurs in exactly one block of $B$. The pair $(X, B)$ is a Steiner Triple System of $u$ varieties denoted STS $(u)$.

Proposition $26\left(\mathrm{see}^{6}\right)$. An STS $(u)$ exists if and only if $u \equiv 1(\bmod 6)$ or $u \equiv 3(\bmod 6)$.

Definition 27. The replication number $r$ of an STS $u$ is the number of blocks that contain a fixed variety.

It can be shown ${ }^{7}$, for an STS $(u)$, that the replication number $r$ satisfies $r=\frac{u-1}{2}$, regardless of the variety chosen. Similarly, the number of blocks is exactly $b=\frac{u(u-1)}{6}$.

Steiner Triple Systems will provide the basis for constructing $(1,2,3)$ matrices. However, they exist only for a limited number of varieties. Thus we consider an alternative structure to cover some of the remaining cases. 
Definition 28. Let $X=\{1,2, \ldots, u\}$ for $u \geq 3$ (these elements are called varieties). Let $K$ be a non-empty set of natural numbers. Let $B$ be a set of non-empty subsets of $X$ called blocks, such that for each $b \in B,|b| \in K$. In addition, each pair of the $u$ elements of $X$ must appear in exactly one block of $B$. The pair $(X, B)$ is a $(u, K, 1)$ Pairwise Balanced Design, also denoted by $(u, K, 1)$-PBD.

Note that the key difference between an $S T S(u)$ and a $P B D(u, K, 1)$ is that the sizes of the blocks in a $P B D(u, K, 1)$ may vary, while an $S T S(u)$ always has blocks of size 3.

Definition 29. Let $D=(P, B)$ where $P=\left\{p_{1}, p_{2}, p_{3}, \ldots, p_{u}\right\}$ is a set of $u$ varieties and $B=\left\{b_{1}, b_{2}, b_{3}, \ldots, b_{v}\right\}$ is a set of subsets of $P$. The incidence matrix of $D$ is a $|B| \times|P|$ binary matrix $M$ in which each entry $M_{i j}$ contains a 1 if subset $b_{i}$ contains variety $p_{j}$ and a 0 if subset $b_{i}$ does not contain variety $p_{j}$.

Example 30. Figure 7 shows the incidence matrix of an STS(7) with $X=\{1,2,3,4,5,6,7\}$ and

$$
B=\{\{1,2,4\},\{2,3,5\},\{3,4,6\},\{4,5,7\},\{5,6,1\},\{6,7,2\},\{7,1,3\}\} .
$$

\begin{tabular}{|l|lllllll|}
\hline & 1 & 2 & 3 & 4 & 5 & 6 & 7 \\
\hline$b_{1}$ & 1 & 1 & 0 & 1 & 0 & 0 & 0 \\
\hline$b_{2}$ & 0 & 1 & 1 & 0 & 1 & 0 & 0 \\
\hline$b_{3}$ & 0 & 0 & 1 & 1 & 0 & 1 & 0 \\
\hline$b_{4}$ & 0 & 0 & 0 & 1 & 1 & 0 & 1 \\
\hline$b_{5}$ & 1 & 0 & 0 & 0 & 1 & 1 & 0 \\
\hline$b_{6}$ & 0 & 1 & 0 & 0 & 0 & 1 & 1 \\
\hline$b_{7}$ & 1 & 0 & 1 & 0 & 0 & 0 & 1 \\
\hline
\end{tabular}

Figure 7. The incidence matrix of an STS(7).

Note that all parameters of the STS $(u)$ are visible in this matrix. Each row represents one block of size 3, and so each row (that is, a block) has exactly three 1's in it. Every pair of varieties appears in exactly one block, and so every pair of columns (that is, varieties) share a 1 in exactly one position. Finally, every variety is in exactly 3 blocks, and so every column contains exactly three 1's as well.

Example 31. Figure 8 shows the incidence matrix of an $(11,\{3,5\}, 1)-\mathrm{PBD}$.

\begin{tabular}{|l|lllllllllll|}
\hline & 1 & 2 & 3 & 4 & 5 & 6 & 7 & 8 & 9 & 10 & 11 \\
\hline$b_{1}$ & 1 & 1 & 1 & 1 & 1 & 0 & 0 & 0 & 0 & 0 & 0 \\
\hline$b_{2}$ & 1 & 0 & 0 & 0 & 0 & 1 & 1 & 0 & 0 & 0 & 0 \\
\hline$b_{3}$ & 1 & 0 & 0 & 0 & 0 & 0 & 0 & 1 & 0 & 0 & 1 \\
\hline$b_{4}$ & 1 & 0 & 0 & 0 & 0 & 0 & 0 & 0 & 1 & 1 & 0 \\
\hline$b_{5}$ & 0 & 1 & 0 & 0 & 0 & 1 & 0 & 1 & 0 & 0 & 0 \\
\hline$b_{6}$ & 0 & 1 & 0 & 0 & 0 & 0 & 1 & 0 & 1 & 0 & 0 \\
\hline$b_{7}$ & 0 & 1 & 0 & 0 & 0 & 0 & 0 & 0 & 0 & 1 & 1 \\
\hline$b_{8}$ & 0 & 0 & 1 & 0 & 0 & 1 & 0 & 0 & 1 & 0 & 0 \\
\hline$b_{9}$ & 0 & 0 & 1 & 0 & 0 & 0 & 1 & 0 & 0 & 0 & 1 \\
\hline$b_{10}$ & 0 & 0 & 1 & 0 & 0 & 0 & 0 & 1 & 0 & 1 & 0 \\
\hline$b_{11}$ & 0 & 0 & 0 & 1 & 0 & 1 & 0 & 0 & 0 & 1 & 0 \\
\hline$b_{12}$ & 0 & 0 & 0 & 1 & 0 & 0 & 1 & 1 & 0 & 0 & 0 \\
\hline$b_{13}$ & 0 & 0 & 0 & 1 & 0 & 0 & 0 & 0 & 1 & 0 & 1 \\
\hline$b_{14}$ & 0 & 0 & 0 & 0 & 1 & 1 & 0 & 0 & 0 & 0 & 1 \\
\hline$b_{15}$ & 0 & 0 & 0 & 0 & 1 & 0 & 1 & 0 & 0 & 1 & 0 \\
\hline$b_{16}$ & 0 & 0 & 0 & 0 & 1 & 0 & 0 & 1 & 1 & 0 & 0 \\
\hline
\end{tabular}

Figure 8. The incidence matrix of an $(11,\{3,5\}, 1)-\mathrm{PBD}$. 
These incidence matrices will be the starting point from which we create $(1,2,3)$ matrices that will ultimately represent our guessing games.

Lemma 32. Let $n \equiv 1$ or $n \equiv 3(\bmod 6)$ and $2 \leq m \leq \frac{n(n-1)}{6}+1$. Suppose $c=(1,1, \ldots, 1)$. Then there exists an $m \times n$ $(1,2,3)$ matrix $[G]$ which is a valid guessing game matrix for an $(m, c, 1)$ guessing game $G$ with exactly $n$ questions.

Proof. Let $n \equiv 1(\bmod 6)$ or $n \equiv 3(\bmod 6)$. By Proposition 26 , there exists a STS $(n) S$ with varieties $N=\{1,2,3, \ldots, n-$ $1, n\}$, block set $B$ such that $|B|=\frac{n(n-1)}{6}$, and replication number $r$ such that $r=\frac{n-1}{2}$. We will use $B$ to construct a new set of blocks.

We begin by removing variety $n$ from all blocks in $B$ and adding a single block $\{n\}$. More precisely, let $B^{\prime}=\{b \backslash\{n\} \mid b \in$ $B\} \cup\{\{n\}\}$. Then $B^{\prime}$ contains $\frac{n(n-1)}{6}-r$ (unchanged) blocks of weight $3, r=\frac{n-1}{2}$ blocks of weight 2 , and a single block of weight 1 .

Let $\left[S^{\prime}\right]$ be the incidence matrix of $S^{\prime}=\left(N, B^{\prime}\right)$. We will show that $d\left(\left[S^{\prime}\right]\right) \geq 3$.

Our key observation comes from the fact that every pair of varieties in $N$ appears in exactly one block in $B$. Thus in $B$, every pair of blocks differ by at least 4 elements. In terms of $[S]$, every pair of rows differs in at least four coordinates, meaning that the minimum distance of $[S]$ is at least 4 . Because any two rows in $\left[S^{\prime}\right]$ of weight 3 correspond directly to blocks of $S$, these two rows must have distance at least 4 .

Let $\mathbf{v}_{i}$ and $\mathbf{v}_{j}$ be two rows of $\left[S^{\prime}\right]$ such that $\left|\mathbf{v}_{i}\right|=3$ and $\left|\mathbf{v}_{j}\right|=2$. Based on a similar observation, vectors $\mathbf{v}_{i}$ and $\mathbf{v}_{j}$ share at most one 1 in the same coordinate. Thus, $\mathrm{d}\left(\mathbf{v}_{i}, \mathbf{v}_{j}\right) \geq 3$.

Next consider two rows $\mathbf{v}_{i}$ and $\mathbf{v}_{j}$ of $\left[S^{\prime}\right]$ with $\left|\mathbf{v}_{i}\right|=2$ and $\left|\mathbf{v}_{j}\right|=2$. By construction, the corresponding blocks $b_{i}$ and $b_{j}$ of $S$ both originally contained the variety $n$. Therefore $b_{i}$ and $b_{j}$ do not share any variety other than $n$. Thus, $\mathbf{v}_{i}$ and $\mathbf{v}_{j}$ share 1's in no coordinates. It follows that $\mathrm{d}\left(\mathbf{v}_{i}, \mathbf{v}_{j}\right)=4$. In particular, the rows of weight 2 in $\left[S^{\prime}\right]$ contain a single 1 in every column other than the column corresponding to variety $n$, which has only 0 's.

Finally, because variety $n$ has been removed from all blocks in $B^{\prime}$, the distance between any row $\mathbf{v}_{i}$ and the row $\mathbf{v}_{n}$ containing only variety $n$ must be at least 3 .

Thus, $\mathrm{d}\left(\left[S^{\prime}\right]\right) \geq 3$. In addition, $\left[S^{\prime}\right]$ contains one row with a weight of $1, \frac{n-1}{2}$ rows with weight 2 , and the remaining $\frac{n(n-1)}{6}-\frac{n-1}{2}$ rows have weight 3 , for a total of $\frac{n(n-1)}{6}+1$ rows. Thus $\left[S^{\prime}\right]$ forms a valid guessing game matrix for a $\left(\frac{n(n-1)}{6}+1, c, 1\right)$ guessing game.

The construction above explains how to construct a matrix with exactly $m=\frac{n(n-1)}{6}+1$ rows. To create a valid matrix for a game with $m<\frac{n(n-1)}{6}+1$, we simply remove as many rows of $\left[S^{\prime}\right]$ as necessary, beginning with rows of weight 3 . This cannot affect the minimum distance of the matrix.

Next, we consider a case for which a Steiner Triple System does not exist.

Proposition $33\left(\right.$ See Lindner $\left.^{6}\right)$. For $n \equiv 5(\bmod 6)$, there exists an $(n,\{3,5\}, 1)$-PBD $D$ such that $D$ has exactly one block of size 5 while the rest of the blocks have size 3 .

Lemma 34. Let $n \equiv 5(\bmod 6)$ and $2 \leq m \leq \frac{n^{2}-n-2}{6}$. Suppose $c=(1,1, \ldots, 1)$. Then there exists an $m \times n(1,2,3)$ matrix $[G]$ which is a valid guessing game matrix for an $(m, c, 1)$ guessing game $G$ with exactly $n$ questions.

Proof. Let $n \equiv 5(\bmod 6)$. By Proposition 33, there exists a $(n,\{3,5\}, 1)$-PBD $D$ with varieties $N=\{1,2, \ldots, n\}$ and block set $B$ that contains exactly one block of size 5 while the rest of the blocks have size 3 . By counting pairs, we can determine that $D$ contains $\frac{\left(\begin{array}{l}n \\ 2\end{array}\right)-\left(\begin{array}{l}5 \\ 2\end{array}\right)}{\left(\begin{array}{l}3 \\ 2\end{array}\right)}=\frac{n^{2}-n-20}{6}$ blocks of weight 3 . Without loss of generality, we assume that $\{n, b, c, d, e\}$ is the block of weight 5 in $D$.

We now create a new block set by "breaking apart" the block of size 5. This will be similar to the proof of Lemma 32, but with the added complication of the block of size 5 . 
Let $P=\{b \in B \mid n \in b$ and $|b|=3\}$ be the set of triples in $D$ containing the variety $n$, and notice that $|P|=\frac{n-5}{2}$. Define $P^{\prime}=\{b \backslash\{n\} \mid b \in P\}$. Let $T=\{b \in B \mid n \notin b$ and $|b|=3\}$ be the set of triples in $D$ not containing the variety $n$. We leave $T$ unchanged.

We construct a new set of blocks $B^{\prime}=P^{\prime} \cup T \cup\{\{b, c\},\{d, e\},\{n\}\}$. Note that $\{\{b, c\},\{d, e\},\{n\}\}$ is essentially a "factoring” of the block of size 5 . Then the set $B^{\prime}$ contains $\frac{\left(\begin{array}{c}n \\ 2\end{array}\right)-\left(\begin{array}{c}5 \\ 5\end{array}\right)}{\left(\begin{array}{c}3 \\ 2\end{array}\right)}+3=\frac{n^{2}-n-2}{6}$ blocks: $\frac{\left(\begin{array}{c}n \\ 2\end{array}\right)-\left(\begin{array}{c}5 \\ 2\end{array}\right)}{\left(\begin{array}{l}3 \\ 2\end{array}\right)}-\frac{n-5}{2}$ of weight 3 from $T, \frac{n-5}{2}$ of weight 2 from $P^{\prime}$, two new blocks also of weight 2 , and the new block of weight 1 . We note that $B^{\prime}$ contains $\frac{n-5}{2}+2=\frac{n-1}{2}$ blocks of size 2 , the maximum possible number of such blocks.

The resulting structure $D^{\prime}=\left(N, B^{\prime}\right)$ that has been constructed from $D$ will have an incidence matrix $\left[D^{\prime}\right]$ in the form shown in Figure 9.

\begin{tabular}{|ccccccc|c|}
\hline & & & & & & & 0 \\
& & & A & & & & $\vdots$ \\
& & & & & & & 0 \\
\hline 1 & 1 & 0 & 0 & $\cdots$ & 0 & 0 & 0 \\
0 & 0 & 1 & 1 & $\cdots$ & 0 & 0 & 0 \\
& & & & $\vdots$ & & & 0 \\
0 & 0 & 0 & 0 & $\cdots$ & 1 & 1 & 0 \\
\hline 0 & 0 & 0 & 0 & $\cdots$ & 0 & 0 & 1 \\
\hline
\end{tabular}

Figure 9. The incidence matrix $\left[D^{\prime}\right]$.

Similarly to the proof of Lemma 32, by using the fact that every pair in $N$ appears in exactly one block of $D$, it follows that $\mathrm{d}\left(\left[D^{\prime}\right]\right) \geq 3$. Since we have shown that $\left[D^{\prime}\right]$ contains one row with a weight of $1, \frac{n-1}{2}$ rows with weight 2 which are mutually disjoint, while the remaining rows have weight 3 and $\mathrm{d}\left(D^{\prime}\right) \geq 3$, it follows that $\left[D^{\prime}\right]$ is a valid $(1,2,3)$ guessing game matrix for a $\left(\frac{n^{2}-n-2}{6}, c, 1\right)$ guessing game with $n$ questions.

The construction above explains how to construct a matrix with exactly $m=\frac{n^{2}-n-2}{6}$ rows. To create a valid matrix for a game with $m<\frac{n^{2}-n-2}{6}$, we remove as many rows as necessary, beginning with rows of weight 3 . This cannot affect the minimum distance of the matrix.

Theorem 35. Let $c=(1,1, \ldots, 1)$ and let $n \geq 3$. Suppose that one of the following is true:

- $n \equiv 1$ or $3(\bmod 6)$ and $2 \leq m \leq \frac{n(n-1)}{6}+1$

- $n \equiv 5(\bmod 6)$ and $2 \leq m \leq \frac{n^{2}-n-2}{6}$

Then a $(1,2,3)$ matrix exists and provides the cheapest possible cost for an $(m, c, 1)$ guessing game with exactly $n$ questions.

Proof. Under the assumptions on $n$, an $m \times n(1,2,3)$ matrix $[M]$ exists by Lemmas 32 and 34 . Note that if $m$ is not as large as possible, then some rows of weight 3 must be removed from the corresponding $(1,2,3)$ matrix, as described in the proof of the respective Lemma. By Theorem 24, $[M]$ gives the cheapest possible cost for an $(m, c, 1)$ guessing game with exactly $n$ questions.

We note that Theorem 35 covers the interesting case where $m \leq \frac{n-1}{2}+1$, in which case a $(1,2,3)$ matrix $[M]$ contains only rows of weight 2 and 1 (because, per the definition, all rows of weight 3 must be removed before removing any rows of lower weight). In this case, $[M]$ is the same as a $(2,1)$ matrix, although possibly with several extra columns containing all O's (effectively, questions that are unused). Thus Theorem 35 is consistent with the conclusion of Theorem 21 applied with $c=(1,1, \ldots, 1)$. In this case, the restriction on the number of questions has no effect. 
Example 36. The following is the construction of a $(1,2,3)$ matrix with $m=8$. We begin with the incidence matrix of an STS(7) from Figure 7. By following the construction of a $(1,2,3)$ matrix as described in Lemma 32, we let the variety 1 be the variety that we remove from the blocks and then replace in a block of weight 1 . From this, we yield the matrix in Figure 10.

\begin{tabular}{|l|lllllll|}
\hline & 1 & 2 & 3 & 4 & 5 & 6 & 7 \\
\hline$b_{1}$ & 0 & 1 & 0 & 1 & 0 & 0 & 0 \\
\hline$b_{2}$ & 0 & 1 & 1 & 0 & 1 & 0 & 0 \\
\hline$b_{3}$ & 0 & 0 & 1 & 1 & 0 & 1 & 0 \\
\hline$b_{4}$ & 0 & 0 & 0 & 1 & 1 & 0 & 1 \\
\hline$b_{5}$ & 0 & 0 & 0 & 0 & 1 & 1 & 0 \\
\hline$b_{6}$ & 0 & 1 & 0 & 0 & 0 & 1 & 1 \\
\hline$b_{7}$ & 0 & 0 & 1 & 0 & 0 & 0 & 1 \\
\hline$b_{8}$ & 1 & 0 & 0 & 0 & 0 & 0 & 0 \\
\hline
\end{tabular}

Figure 10. The incidence matrix with variety 1 removed and a new row added.

By rearranging the columns and rows of the matrix in Figure 10, we obtain the $(1,2,3)$ matrix in Figure 11.

\begin{tabular}{|l|lllllll|}
\hline & 2 & 4 & 3 & 7 & 5 & 6 & 1 \\
\hline$b_{2}$ & 1 & 0 & 1 & 0 & 1 & 0 & 0 \\
\hline$b_{3}$ & 0 & 1 & 1 & 0 & 0 & 1 & 0 \\
\hline$b_{4}$ & 0 & 1 & 0 & 1 & 1 & 0 & 0 \\
\hline$b_{6}$ & 1 & 0 & 0 & 1 & 0 & 1 & 0 \\
\hline$b_{1}$ & 1 & 1 & 0 & 0 & 0 & 0 & 0 \\
\hline$b_{7}$ & 0 & 0 & 1 & 1 & 0 & 0 & 0 \\
\hline$b_{5}$ & 0 & 0 & 0 & 0 & 1 & 1 & 0 \\
\hline$b_{8}$ & 0 & 0 & 0 & 0 & 0 & 0 & 1 \\
\hline
\end{tabular}

Figure 11. The final $(1,2,3)$ matrix.

If $c=(1,1,1,1,1,1,1)$, this gives a $(8, c, 1)$ guessing game with exactly 7 questions and total cost $3 \cdot 8-\left\lfloor\frac{8-1}{2}\right\rfloor-2=19$. Example 37. The following is the construction of a $(1,2,3)$ matrix with $m=11$ as described in this section. We begin with the incidence matrix of an $(11,\{3,5\}, 1)$-PBD from Figure 8.

By following the construction of a $(1,2,3)$ matrix as described in Lemma 34, we let the variety 1 be the variety that we remove from the blocks and then replace in a block of weight 1 . From this, we yield the matrix seen in Figure 12. The blocks created from original block of weight 5 are highlighted. 


\begin{tabular}{|l|lllllllllll|}
\hline & 1 & 2 & 3 & 4 & 5 & 6 & 7 & 8 & 9 & 10 & 11 \\
\hline$b_{1}$ & 0 & 1 & 1 & 0 & 0 & 0 & 0 & 0 & 0 & 0 & 0 \\
\hline$b_{2}$ & 0 & 0 & 0 & 1 & 1 & 0 & 0 & 0 & 0 & 0 & 0 \\
\hline$b_{3}$ & 0 & 0 & 0 & 0 & 0 & 1 & 1 & 0 & 0 & 0 & 0 \\
\hline$b_{4}$ & 0 & 0 & 0 & 0 & 0 & 0 & 0 & 1 & 0 & 0 & 1 \\
\hline$b_{5}$ & 0 & 0 & 0 & 0 & 0 & 0 & 0 & 0 & 1 & 1 & 0 \\
\hline$b_{6}$ & 0 & 1 & 0 & 0 & 0 & 1 & 0 & 1 & 0 & 0 & 0 \\
\hline$b_{7}$ & 0 & 1 & 0 & 0 & 0 & 0 & 1 & 0 & 1 & 0 & 0 \\
\hline$b_{8}$ & 0 & 1 & 0 & 0 & 0 & 0 & 0 & 0 & 0 & 1 & 1 \\
\hline$b_{9}$ & 0 & 0 & 1 & 0 & 0 & 1 & 0 & 0 & 1 & 0 & 0 \\
\hline$b_{10}$ & 0 & 0 & 1 & 0 & 0 & 0 & 1 & 0 & 0 & 0 & 1 \\
\hline$b_{11}$ & 0 & 0 & 1 & 0 & 0 & 0 & 0 & 1 & 0 & 1 & 0 \\
\hline$b_{12}$ & 0 & 0 & 0 & 1 & 0 & 1 & 0 & 0 & 0 & 1 & 0 \\
\hline$b_{13}$ & 0 & 0 & 0 & 1 & 0 & 0 & 1 & 1 & 0 & 0 & 0 \\
\hline$b_{14}$ & 0 & 0 & 0 & 1 & 0 & 0 & 0 & 0 & 1 & 0 & 1 \\
\hline$b_{15}$ & 0 & 0 & 0 & 0 & 1 & 1 & 0 & 0 & 0 & 0 & 1 \\
\hline$b_{16}$ & 0 & 0 & 0 & 0 & 1 & 0 & 1 & 0 & 0 & 1 & 0 \\
\hline$b_{17}$ & 0 & 0 & 0 & 0 & 1 & 0 & 0 & 1 & 1 & 0 & 0 \\
\hline$b_{18}$ & 1 & 0 & 0 & 0 & 0 & 0 & 0 & 0 & 0 & 0 & 0 \\
\hline
\end{tabular}

Figure 12

By rearranging the columns and rows of the matrix in Figure 12, we obtain the $(1,2,3)$ matrix in Figure 13.

\begin{tabular}{|c|ccccccccccc|}
\hline & 2 & 3 & 4 & 5 & 6 & 7 & 8 & 11 & 9 & 10 & 1 \\
\hline$b_{6}$ & 1 & 0 & 0 & 0 & 1 & 0 & 1 & 0 & 0 & 0 & 0 \\
\hline$b_{7}$ & 1 & 0 & 0 & 0 & 0 & 1 & 0 & 0 & 1 & 0 & 0 \\
\hline$b_{8}$ & 1 & 0 & 0 & 0 & 0 & 0 & 0 & 1 & 0 & 1 & 0 \\
\hline$b_{9}$ & 0 & 1 & 0 & 0 & 1 & 0 & 0 & 0 & 1 & 0 & 0 \\
\hline$b_{10}$ & 0 & 1 & 0 & 0 & 0 & 1 & 0 & 1 & 0 & 0 & 0 \\
\hline$b_{11}$ & 0 & 1 & 0 & 0 & 0 & 0 & 1 & 0 & 0 & 1 & 0 \\
\hline$b_{12}$ & 0 & 0 & 1 & 0 & 1 & 0 & 0 & 0 & 0 & 1 & 0 \\
\hline$b_{13}$ & 0 & 0 & 1 & 0 & 0 & 1 & 1 & 0 & 0 & 0 & 0 \\
\hline$b_{14}$ & 0 & 0 & 1 & 0 & 0 & 0 & 0 & 1 & 1 & 0 & 0 \\
\hline$b_{15}$ & 0 & 0 & 0 & 1 & 1 & 0 & 0 & 1 & 0 & 0 & 0 \\
\hline$b_{16}$ & 0 & 0 & 0 & 1 & 0 & 1 & 0 & 0 & 0 & 1 & 0 \\
\hline$b_{17}$ & 0 & 0 & 0 & 1 & 0 & 0 & 1 & 0 & 1 & 0 & 0 \\
\hline$b_{1}$ & 1 & 1 & 0 & 0 & 0 & 0 & 0 & 0 & 0 & 0 & 0 \\
\hline$b_{2}$ & 0 & 0 & 1 & 1 & 0 & 0 & 0 & 0 & 0 & 0 & 0 \\
\hline$b_{3}$ & 0 & 0 & 0 & 0 & 1 & 1 & 0 & 0 & 0 & 0 & 0 \\
\hline$b_{4}$ & 0 & 0 & 0 & 0 & 0 & 0 & 1 & 1 & 0 & 0 & 0 \\
\hline$b_{5}$ & 0 & 0 & 0 & 0 & 0 & 0 & 0 & 0 & 1 & 1 & 0 \\
\hline$b_{18}$ & 0 & 0 & 0 & 0 & 0 & 0 & 0 & 0 & 0 & 0 & 1 \\
\hline
\end{tabular}

Figure 13. The final $(1,2,3)$ matrix. 


\section{ACKNOWLEDGEMENTS}

Thank you to the Student Summer Scholars (S3) program and at Grand Valley State University and all of the program's wonderful donors for funding this research.

The authors also thank the anonymous referees whose suggestions helped to improve and streamline this paper.

\section{REFERENCES}

1. Ulam, S. M. (1976) Adventures of a Mathematician, Scribner, New York.

2. Spencer, J. (1984) Guess a number - with lying, Math. Mag. 57, 105-108.

3. Deppe, C. (2007) Coding with feedback and searching with lies, in entropy, search, complexity (Csiszár, I., Katona, G., Tardos, G., Eds.), Bolyai Society Mathematical Studies vol. 16, 27-70, Springer.

4. Pelc, A. (2002) Searching games with errors: fifty years of coping with liars, Theoret. Comput. Sci. 270, 71-109.

5. Huffman, W. and Pless, V. (2003) Fundamentals of Error-Correcting Codes, Cambridge University Press.

6. Lindner, C. (2008) Design Theory 2nd ed., Chapman and Hall/CRC.

7. Brualdi, R. (2009) Introductory Combinatorics, Pearson.

\section{ABOUT THE STUDENT AUTHOR}

Lindsay Czap completed this project while earning a bachelor's degree in mathematics from Grand Valley State University with funding from the Student Summer Scholars program. She is currently a graduate student at Middle Tennessee State University earning her master's degree in pure mathematics. She plans to pursue her doctoral studies in undergraduate mathematics education.

\section{PRESS SUMMARY}

The game "20 questions" has always been a staple for children trapped in long car rides. We explore guessing games like "20 questions" in which one player chooses a number, and the other player asks yes-or-no questions to determine the number. In our games, the responding player may lie once. By adding a cost to each question in these games, we are able to quantify their efficiency. We determine the most efficient games in two cases: When the number of questions is unlimited, and when the number of questions is limited and the costs are especially simple. In each case, we explain exactly how to play these games in the most efficient way. 


\title{
Analysis of Fungal Contamination on Commercially Sold Rice in Puerto Rico
}

\author{
Nicole Colón Carrión, Chad Lozada Troche \\ Department of Biology, University of Puerto Rico, Cayey, PR \\ Student: nicole.colon11@upr.edu \\ Mentor:chad.lozada@upr.edu
}

\begin{abstract}
Crops and stored grains are susceptible to pathogens that represent a threat to our health. The study presented herein compares the normal surface and endophytic fungal communities present on white and brown rice grains. One hundred grains of each rice variety was analyzed to determine their fungal contaminants and endophytes. Fungi were inoculated on SDA media, and purified in PDA media; morphological characterization was performed followed by amplification of the ITS region using PCR for all fungal isolates. Statistical analysis indicated significant differences between medium brown rice compared to white rice for surface and endophytic communities ( $\mathrm{p}$-value $\leq 0.05$ ). In addition, a higher fungal diversity was found on brown rice grains compared to white rice. This variation may be due to differences in the processing methods used for each rice grain type. BLAST analysis revealed the presence of toxigenic strains of Aspergillus flavus, A.oryzae, Penicillium verrucosum, and P. viridicatum. The study of fungal growth in rice grains can contribute to the minimization of mycotoxin production by its prevention and control; therefore, decreasing crop contamination and human exposure to their metabolites.
\end{abstract}

\section{KEYWORDS}

Fungi; Rice; Fungal Contaminants; Fungal Endophytes

\section{INTRODUCTION}

For years, rice has been one of the most important crops worldwide, not only for its nutritional value but also for the economic profit generated by its production and consumption. Produce and commercialized in approximately more than 25 countries, rice has become an essential nutrient in our daily diet. According to the Food and Agriculture Organization, in 2015 milled rice production reached approximately 491.5 million tons (milled eq.); with a feed use of 1.8 million tons (milled eq.). ${ }^{1}$ China, India, Indonesia, Bangladesh, Vietnam, and Thailand are described as the six major producers of this crop. Even though the United States production of rice is relatively low compared to the six top producers, it is still considered a major one. In 2015, U.S. rice exports increased 18\% from the prior year to a total of 3.5 million tons, the highest in 4 years; most of the yield was exported to Latin America. ${ }^{2}$ Puerto Rico imports $85 \%$ of their food products from the United States, including rice, based on the U.S. merchant marine acts, under the Foraker Act. This law states that entrance of every product into the island must be carried on U.S. shipments. ${ }^{3}$

Rice crops can be contaminated with mycotoxin-producing fungi. These pathogens are known to infest rice via two pathways: (1) in the field while rice is growing (field fungi); pre-harvest method, or (2) during the processing and storage after harvesting (storage fungi); post-harvest method. The presence or absence of mycotoxins depends on different factors such as genotype of the fungal isolate, interactions with other organisms on the substrate where they coexist, origin of the crop and its climatology, stress factors, available nutrition, physical damage due to insect activity, or agricultural and post-harvest practices. ${ }^{4,5}$ The major mycotoxins, based on the levels of toxicity, have been identified as aflatoxins, ochratoxins, fumonisins and trichothecenes. ${ }^{6}$ These can be produced by various genera including Aspergillus, Penicillium, Fusarium, Claviceps, Stachybotrys. ${ }^{6}$ Human exposure to mycotoxin has been linked to many neurological diseases, immunosuppression, carcinogenic effects, nephrotoxicity, and hepatotoxicity, among others. ${ }^{6-8}$

It is estimated that approximately $25 \%$ of the world cereal production is contaminated with mycotoxins or their derivatives. ${ }^{9}$ The $^{-}$ study presented here is an initial effort to analyze endophytic and surface fungal contamination in milled white rice grains and brown rice grains commercially sold in Puerto Rico. Due to the level of importance, this crop possesses to human nutritional sustainability, the study of mycotoxin-producing fungi will provide insight for future prevention and control of exposure to mycotoxins, therefore decreasing global economic losses, crop contamination, and human exposure to fungal pathogens and their secondary metabolites. 


\section{METHODS AND PROCEDURES}

\section{Collection of Rice Grains}

Between 454 and 907 grams of milled rice samples were purchased from stores and supermarkets located in Caguas, Puerto Rico; standard packages sold for medium fortified brown rice contained less grain quantity (454 grams) when compared with medium fortified white rice (907 grams). Multiple batches were obtained at two-time points: August 2015 and June 2017, and analyzed one week after purchase; bags were stored at $25^{\circ} \mathrm{C}$, and ambient light. Once open, batches were not re-used or stored. Two different types of rice, medium fortified white and brown rice, were tested for surface and endophytic fungal isolates. Each group consisted of biological triplicates containing 50 grains per rice type. Both samples were selected from the same brand denominated as Brand A.

\section{Surface Fungal Isolation Assay}

Fungal Isolation was performed following Samson et al. (2002) protocol. ${ }^{10}$ A total of 150 grains per rice type were selected for analysis; $n=3,50$ samples per $n$. Grains were immersed in a solution of $0.4 \%$ of sodium hypochlorite for 2 min, rinsed with $\mathrm{dH}_{2} \mathrm{O}$, and dried for sterilization. Direct plating of grains was performed into Sabouraud Dextrose Agar (SDA) and incubated at $25^{\circ} \mathrm{C}$ for 7 days and ambient light; 5 particles per plate. Fungal colonies were sub-cultured and purified on Potato Dextrose Agar (PDA) under the same conditions.

\section{Endophyte Isolation Assay}

Fungal Isolation was carried out using a modification of the method described by Samson et al. (2002)..$^{10}$ One hundred fifty grains per rice type were selected for analysis; $n=3,50$ samples per $n$. Grains were half-cut and surface-sterilized with a solution of $0.4 \%$ of sodium hypochlorite for 2 min, rinsed with $\mathrm{dH}_{2} \mathrm{O}$ and dried. Plating of samples and purification of isolates was performed as described above.

\section{Control Assay}

Ten grains were surface-sterilized as described previously, dried, and autoclaved. Plating of samples and purification of isolates was performed as described above.

\section{Morphological Identification of Isolates}

Fungal characterization for surface and endophytic isolates was performed following Lozada-Troche et al. (1998) "Moist chambers" protocol. ${ }^{11}$ Chamber cultures consisted of Petri dishes containing a $75 \mathrm{~mm}$ microscopic slide placed above a V-shaped glass rod with a portion of SDA media over the slide and filled with $\mathrm{dH}_{2} \mathrm{O}$. Mycelia of isolates were transfer into the chamber's media and a cover with a glass slip. Chambers were maintained at room temperature and ambient light. After 96hrs, fungal structures were stained with lactophenol cotton blue to identify the genera according to their hypha, conidia and spore morphology. Isolates were identified following the Dugan (2006) identification guide. ${ }^{12}$

\section{DNA Extraction}

Extraction and purification of DNA from fungal isolates were carried out using a modification of the protocol described by Liu $e t$ al. (2000). ${ }^{13}$ Hyphal tip transfer of samples was performed into SDA liquid media and incubated for 7 days at $25^{\circ} \mathrm{C}$ with a shaking speed of $40 \mathrm{rmp}$. Fungal isolates were centrifuged at $10,000 \mathrm{x} g$ for five minutes, and the supernatant discarded. Five hundred $\mu \mathrm{l}$ of lysis buffer was added to Eppendorf microtubes containing fungal hyphae and blended for ten minutes. Once homogenous, $150 \mu \mathrm{l}$ of $5 \mathrm{M}$ potassium acetate was added, vortexed, and centrifuged at $10,000 \mathrm{x} g$ for one minute. The supernatant was collected, $600 \mu \mathrm{l}$ of $100 \%$ isopropanol added, and tubes were spun at $10,000 \mathrm{x} g$ for two minutes. The resulting DNA pellet was washed using $500 \mu \mathrm{l}$ of $70 \%$ ethanol, then centrifuged, after which ethanol was removed, and residual ethanol was evaporated at $25^{\circ} \mathrm{C}$ for ten minutes. The pellet was re-suspended in $50 \mu \mathrm{l}$ of $10 \mathrm{Mm}$ Tris.

\section{Amplification of ITS region}

The Internal Transcribed Spacer (ITS) region one and two including 5.8S gene were amplified using specific primers ITS1 and ITS4. ${ }^{14}$ PCR amplifications were carried out in a total volume of $50 \mu$ containing $2.5 \mu \mathrm{M}$ of nucleotide mix, 20 pmol of each primer, 2.5 U of Taq Polymerase (Promega, Madison, USA), 1X reaction buffer and 20-50 ng of genomic DNA. Amplifications were performed in an Eppendorf Master Cycler ${ }^{\circledR}$ gradient (Brinkmann Instruments) using 30 cycles with the following parameters: 1 min denaturation at $94^{\circ} \mathrm{C}, 45 \mathrm{sec}$ annealing at $45^{\circ} \mathrm{C}, 1 \mathrm{~min}$ extension at $72^{\circ} \mathrm{C}$, and a final extension of 5 min at $72^{\circ} \mathrm{C}$. PCR amplifications were verified using a $1 \%$ agarose gel with UVIEW (Biorad) under UV light. Length of amplified fragments was determined by comparison with the migration of Lambda Hind III plus marker (Lambda Biotech Inc., St. Louis, USA). PCR amplicons were purified following the Zymoclean Gel DNA Recovery protocol. ${ }^{15}$ 
Sample Sequencing and Isolate Identification

The sense and reverse strands of the PCR products of the fungal strains were sequenced using the Applied Biosystems Big Dye ${ }^{\mathrm{TM}}$ Terminator v3.1 and ABI $3130 \mathrm{xl}$ genetic analyzer (Applied Biosystems/ MDS SCIEX, Foster City, USA). DNA purified samples were sent to the Sequencing and Genomics facility, University of Puerto Rico-Río Piedras for sequencing. The DNA sequence data from fungal amplicons were analyzed with a BLASTn to identify the strains at the species level. ${ }^{16}$ Newly obtained sequences were deposited in GeneBank (Table 1).

\begin{tabular}{ll}
\hline Species & Accession numbers \\
\hline Aspergillus aculeatus & $\mathrm{KY} 006833, \mathrm{KY} 006835, \mathrm{KY} 006836, \mathrm{KY} 006838, \mathrm{KY} 006839$ \\
A. clavatus & $\mathrm{KY} 006827, \mathrm{KY} 006843, \mathrm{KY} 006845, \mathrm{KX} 944171, \mathrm{KX} 944177$ \\
A. flavus & $\mathrm{KY} 006825, \mathrm{KY} 006826, \mathrm{KY} 006828, \mathrm{KY} 006831, \mathrm{KY} 006832, \mathrm{KY} 006844$, \\
& $\mathrm{KX} 944169, \mathrm{KX} 944170, \mathrm{KX} 944180, \mathrm{KX} 944182$ \\
A. japonicus & $\mathrm{KY} 006834$ \\
A. oryzae & $\mathrm{KY} 006837, \mathrm{KY} 006846, \mathrm{KX} 944179$ \\
Fusarium equiseti & $\mathrm{KX} 944173$ \\
Penicillium citrinum & $\mathrm{KX} 944178$ \\
P. polonicum & $\mathrm{KX} 944172, \mathrm{KX} 944174$ \\
P. verrucosum & $\mathrm{KY} 006840, \mathrm{KY} 006841, \mathrm{KY} 006842$ \\
P.viridicatum & $\mathrm{KY} 006830, \mathrm{KX} 944181$ \\
Penicillium sp. & $\mathrm{KY} 006829, \mathrm{KX} 944176$ \\
Rinocladiella similis & $\mathrm{KX} 944175$ \\
\hline
\end{tabular}

Table 1. New ITS sequences obtained in this study. Sequences were deposited in GeneBank; species name and accession number are listed.

\section{Statistical Analysis}

Colonization frequency (CF) was calculated as described by Suryanarayanan and Vijaykrishna (2001); stated in Equation 1. ${ }^{17}$ CF's were graph using Datagrap 4.1 (Visual Data Tools, Inc., 2006). Wilcoxon's test was performed to compare the difference in fungal communities between medium fortified white vs. brown rice for surface and endophytic isolates; $\mathrm{P} \leq 0.05$ was set to determine the significance of results. GraphPad contingency calculator (StataCorp LLC, TX) was used to determine P value.

$$
C F=\left(\frac{\text { number of rice grains colonized by a specific genera }}{\text { number of total rice grains analyzed }}\right) \times 100 \quad \text { Equation } 1 .
$$

\section{RESULTS}

A significant difference in surface and endophytic fungal communities between medium fortified brown vs. white rice Wilcoxon's test was conducted to determine a difference in fungal presence between medium fortified white vs. brown rice. A significant difference between medium brown rice compared to white rice was determined for surface and endophytic communities ( $\mathrm{p}$-value $\leq 0.05$ ) (Table 3). A number of fungal isolates obtain per replicate is presented in table 2 for each group. Control samples showed no fungal colonization.

\begin{tabular}{|c|c|c|c|c|}
\hline Mycoflora Type & Rice Type & N=1 & N=2 & $\mathbf{N = 3}$ \\
\hline Surface & White Rice & 5 & 4 & 2 \\
\hline & Brown Rice & 22 & 38 & 40 \\
\hline & & & & \\
\hline Endophytic & White Rice & 3 & 2 & 30 \\
\hline & Brown Rice & 4 & 37 & 2 \\
\hline
\end{tabular}

Table 2. A number of fungal isolates obtain on rice grains. Each replicate consisted of 50 grains.

\begin{tabular}{|c|c|c|}
\hline Mycoflora Type & & P value \\
\hline Surface & White Rice vs. Brown Rice & 0.0495 \\
\hline Endophytic & White Rice vs. Brown Rice & 0.0463 \\
\hline
\end{tabular}

Table 3. P- value of medium fortified white $v$ s. brown rice for surface and endophytic communities. ( $\mathrm{n}=3$, 50 samples per replicate). 
Colonization frequency for surface communities

Identification of fungal isolates revealed strains of Aspergillus, Penicillium, Rhinocladiella, Mucor, and Fusarium. CF's were significantly higher for brown rice compared to white rice, with a higher frequency of Aspergillus strains (Figure 1). Rhinocladiella strains were only found on white rice, while strains of Fusarium and Mucor on brown rice. Furthermore, a significant amount of fungal isolates was identified as 'non-sporulators' due to their inability to develop a reproductive structure.

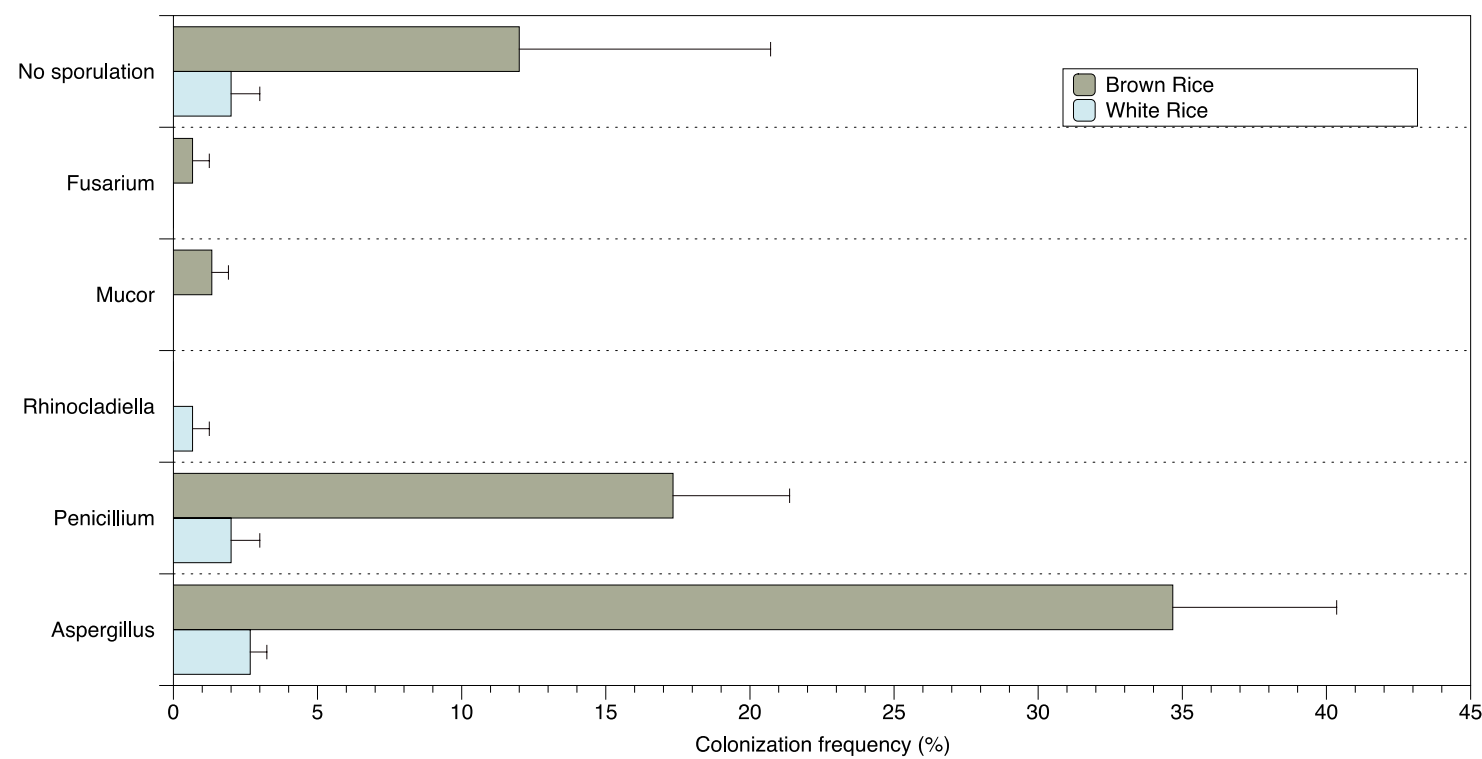

Figure 1. Colonization frequency for surface communities. \% age of CF's on medium fortified white and brown rice grains (three replicates per type).

\section{Blast Sequence Identification}

BLASTn sequence identification was only performed as $\mathrm{n}=1$. Results successfully identified strains of Aspergillus oryzae, Penicillium citrinum and Rinocladiella similis in medium fortified white rice. Strains of Aspergillus clavatus, Aspergillus flavus, Aspergillus oryzae, Fusarium equiseti, Mucor sp., Penicillium alli, Penicillium polonicum, Penicillium viridicatum, and Penicillium sp. were identified in medium brown rice. Endophytes consisted of strains of Aspergillus aculeatus, Aspergillus flavus, Aspergillus japonicas, and Penicillium verrucosum. Percentages of BLAST homology are presented in Table 4.

\begin{tabular}{|c|c|c|c|}
\hline Surface Communities & Rice Type & Endophytic Communities & Rice Type \\
\hline Aspergillus clavatus (99\%) & B & Aspergillus aculeatus (98\%) & W, B \\
\hline Aspergillus flavus (99\%) & B & Aspergillus flavus (96\%) & Aspergillus japonicus (99\%) \\
\hline Aspergillus oryzae (100\%) & W, B & Penicillium verrucosum (99\%) & B \\
\hline Fusarium equiseti (98\%) & B & & \\
\hline Mucor sp. & B & & \\
\hline Penicillium alli (98\%) & B & & \\
\hline Penicillium citrinum (99\%) & W & & \\
\hline Penicillium polonicum $(97 \%)$ & B & & \\
\hline Penicillium viridicatum $(98 \%)$ & B & & \\
\hline Penicillium sp. (99\%) & W & & \\
\hline Rinocladiella similis (98\%) & & & \\
\hline
\end{tabular}

Table 4. Fungal isolates in surface and endophytes mycoflora obtain in this study. Percentage indicates BLAST homology. $\mathrm{W}=$ white medium fortified rice, $\mathrm{B}=$ brown medium rice. ( $\mathrm{n}=1$, three replicates per group). 


\section{DISCUSSION}

Statistical analysis indicated significant differences between medium brown rice compared to white rice for surface and endophytic communities ( $\mathrm{p}$-value $\leq 0.05$ ); suggesting that a higher proportion of medium brown rice contains fungal contaminants. Microscopic fungal identification indicated the presence of genera such as Aspergillus, Penicillium, Rhinocladiella, Mucor, and Fusarium, with CF's ranging from 2\% to 34\% (Figure 1). Furthermore, a higher fungal diversity was observed on brown compared to white rice grains. BLAST sequence identification showed the presence of putative mycotoxin-producing fungi Penicillium citrium in white medium fortified rice, and Aspergillus flavus and Penicillium viridicatum in brown medium fortified rice. The high levels of fungal growth in medium brown rice compared to white rice may be due to harvesting and milling methods. After harvesting, grains undergo a drying and hulling process, at this point, no further processing is necessary for brown rice. However, white rice is subjected to a milling and enriching process which ensures the removal of the outer bran layer and provides the white gloss aspect. ${ }^{18}$ The removal of this layer leads to a loss in protein, vitamins, minerals and lipids levels; utilized by fungi during their nutrition. This may implement a significant reduction of mold's nutrients leading to a reduction in fungal growth and diversity on white rice.

In studies conducted with rice in Cuba and sorghum in Argentina, other researchers found the presence of filamentous and potential mycotoxin-producing genera Aspergillus, Fusarium, and Penicillium, as well as phytopathogenic genera Bipolaris, Curvularia, Alternaria, Pycularia, and Cercospora in rice (Almaguer and Rodriguez-Rajo, 2012), and Alternaria, Aspergillus, Cladosporium, Curvularia, Fusarium, Penicillium, and Phoma in sorghum (Gonzalez et al., 1997). 19, 20 Furthermore, studies conducted with rice in Vietnam revealed the presence of three main genera: Aspergillus, Fusarium, and Penicillium; with focus on toxigenic strains Aspergillus flavus, Aspergillus ochraceus and Penicillium citrinum. ${ }^{21}$ In southern regions of the United States, previous reports showed that Aspergillus flavus and Fusarium verillicoides represent high contaminants for corn samples, while Fusarium species are most common in rice; nevertheless, rice seems to exhibit more resistance to fungal contamination and mycotoxin production than other crops. ${ }^{22}$ Similarly to these studies, our results showed the presence of Aspergillus, Fusarium, and Penicillium genera's in medium fortified white and brown rice; while species analysis revealed the presence of Aspergillus flavus and Penicillium citrinum.

The high occurrence of fungal contamination in commercially sold rice grain in Puerto Rico may be due to poor post-harvesting methods. Extrinsic factors such as humidity, water content, and temperature are a vital factor for fungal infection. Water content is the principal environmental factor that influences the three stages of germination, particularly the first stage: swelling of the spore. ${ }^{23}$ Furthermore, temperatures ranging from $15-40^{\circ} \mathrm{C}$ promote fungal infection. ${ }^{23}$ When imported, grains are prone to constant temperature and humidity changes which contribute to fungal proliferation. Because of the great importance this crop possesses, it is vital that strict pre-harvest and post-harvest methods be applied in order to prevent and reduce the incidence of fungal contamination, and possible mycotoxin production. Thus, the measures applied to reduce and minimize contamination should focus towards the prevention against fungal development in the field, as well as the production of secondary metabolites. ${ }^{4}$

\section{CONCLUSION}

The present study represents an initial effort to reveal the occurrence of surface and endophytic fungal microflora contamination in medium fortified white and brown rice commercially sold in Puerto Rico. The results have shown a higher occurrence of fungal contamination in brown rice grains compared to white rice for surface and endophytic communities. Nevertheless, furthers studies with a higher number of replicates, as well as consideration of other rice brands need to be conducted to establish a specific pattern. Taxonomic results showed a higher fungal diversity on brown rice compared to white rice. In addition, potential mycotoxin-producing species were identified in both white and brown rice grains; Penicillium citrium in white medium fortified rice, and Aspergillus flavus and P. viridicatum in brown medium rice. Further studies should focus on identification, quantification, and prevention of mycotoxins in rice grains. The measures applied to reduce and minimize mycotoxins should focus towards two different aims: the prevention against contamination and fungal development in the cereal, as well as the production of secondary metabolites; and the detoxification of mycotoxins when they occur in the grain. ${ }^{4}$ For the latest, decontamination of mycotoxin and mycotoxin-producers by cooking methods had been successfully implemented. Palavaras et al. (2004) showed a 72-89\% reduction of mycotoxin levels in rice grains after different cooking methods. ${ }^{4,24}$ Hence, autoclaving practices will implement a great and accessible post-harvest method for the reduction of mycotoxin-producers and their metabolites in food samples in industrial settings; as shown in our previous control assay (refer to method section).

\section{ACKNOWLEDGMENTS}

Authors thank Dr. Carlos Betancourt-Lopez for reviewing the manuscript. Authors are also thankful for financial assistance provided by FIDI (Fondo Institucional para el Desarrollo de la Investigación) at the University of Puerto Rico - Cayey, and the Puerto Rico Louis Stokes Alliance for Minority Participation (Grant Number: 1400868). This project was made possible with support from the Sequencing and Genomics Facility of the UPR Río Piedras \& MSRC/UPR, funded by NIH/NIGMS-Award Number P20GM103475. 


\section{REFERENCES}

1. Food and Agriculture Organization, FAO. Rice Market Monitor 2015, http.// wnw.fao.org/economic.RMM (accessed Feb 2016)

2. United States Department of Agriculture Foreign Agricultural Service, U.S.D.A. Rice Outlook 2016, bttp:/ / www.fas.usda.gov/data/grain-world-markets-and-trade (accessed Jan 2016)

3. Bender, L. D. (1998) Economics and business, in The American presence in Puerto Rico 1st ed., 71-77, Publicaciones Puertorriquenas, Puerto Rico.

4. Ferre, F. S. (2016) Worldwide occurrence of mycotoxins in rice. Food Control 62, 291-298. https:// doi.org/10.1016/j.foodcont.2015.10.051

5. Bhat, R., Rai, R. V., \& Karim, A. A. (2010) Mycotoxins in Food and Feed : Present Status and Future Concerns, Compr Rev Food Sci Food Saf 9, 57-81.

6. Serrano, H. A., \& Cardona, N. C. (2015) Micotoxicosis y micotoxinas: generalidades y aspectos básicos, Rev CES Med 29, 143152.

7. Marroquín, A. G., Johnson, N. M., Phillips, T. D., \& Hayes, A. W. (2014) Mycotoxins in a changing global environment - A review. Food Chem Toxicol 69, 220-230. https://doi.org/10.1016/j.fct.2014.04.025

8. Gimeno, A., \& Martins, M. L. (2011) Mycotoxins and mycotoxicoses more significant in animals and humans, in Mycotoxins and mycotoxicoses in animals and humans 3rd ed., 65-81, Special Nutrients, Florida.

9. Aydin, A., Aksu, H., \& Gunsen, U. (2011) Mycotoxin levels and incidence of mould in Turkish rice, Environ Monit Assess 178, 271-280. https:// doi.org/10.1007/s10661-010-1688-9

10. Samson, R. A., Hoekstra E. S., \& Frisvad J. C. (2004) Introduction to food- and airborne fungi 7 th ed., Centraalbureau voor Schimmelcultures, Utrecht.

11. Lozada-Troche C., Ortiz, B., \& Betancourt, C. (1998) Moist chambers, in "Introducción a la Micología Médica. Imprenta Universitaria” 1st ed., 16, Recinto Universitario de Mayagüez, Puerto Rico.

12. Dugan, F. M. (2006) The identification of fungi: an illustrated introduction with keys, glossary, and guide to literature, APS, St. Paul, Min.

13. Liu, D., Coloe, S., Baird, R., \& Pendersen, J. (2000) Rapid mini-preparation of Fungal DNA for PCR, J Clin Microbiol $38,471$.

14. White, T. J., Bruns, T., Lee, S., \& Taylor, J. (1990) Amplification and direct sequencing of fungal ribosomal RNA genes for phylogenetics, in PCR Protocols: a Guide to Methods and Applications (Innis, M. A., Gelfand, D. H., Sninsky, J.J., White, T., Ed.) 1st ed., Academic Press, New York.

15. ZYMO RESEARCH CORP. Zymoclean ${ }^{\mathrm{TM}}$ Gel DNA Recovery Kit Instruction Manual, bttp:// www.zymoresearch.com/downloads/dl/file/id/34/d400li/pdf (accessed Dec 2015)

16. Altschul, S.F., Gish, W., Miller, W., Myers, E. W., \& Lipman D.J. (1990) Basic local alignment search tool, J Mol Biol 215, 403410.

17. Suryanarayanan, T.S., \& Vijaykrishna, D. (2001) Fungal endophytes of aerial roots of Ficus benghalensis, Fungal Divers 8, $155-161$.

18. Yadav, B. K., \& Jindal, V. K. (2001) Monitoring milling quality of rice by image analysis, Comput Electron Agric 33, 19-33.

19. Almaguer, M., Rojas, T. I., \& Rodríguez-rajo, F. J. (2012) Airborne fungal succession in a rice field of Cuba, Eur J Plant Pathol 133, 473-482. https:// doi.org/10.1007/s10658-011-9921-0

20. Mart, E. J., \& Resnik, S. L. (1997) Fungi associated with sorghum grain from Argentina, Mycopathologia 139, 35-41.

21. Trung, T. S., Bailly, J. D., Querin, A., Le Bars, P., \& Guerre, P. (2001). Fungal contamination of rice from South Vietnam, mycotoxinogenesis of selected strains and residues in rice. Revue de Médecine Vétérinaire, 152(7), 555-560.

22. Abbas, H. K., \& Shier, W. T. (2009). Mycotoxin contamination of agricultural products in the southern United States and approaches to reducing it from pre-harvest to final food products. Mycotoxin prevention and control in agriculture. Washington, DC: American Chemical Society, 37-58.

23. Gottlieb, D. (1950) The physiology of spore germination in fungi, Bot Rev 16, 229-257.

24. Palavras, C., Simionato, E., Sylos, M. R. S., and De, C. M. (2004). Effect of cooking on the levels of AFB1 and ochratoxin A in rice. Brazilian J. Food Technol. 7(2):167-171.

\section{ABOUT THE STUDENT AUTHOR}

Nicole Colón Carrión graduated in June 2016 from the University of Puerto Rico Cayey Campus with a bachelors degree in Biology. She has been working along with Dr. Lozada in this research project for two years; where she was awarded two research fellowship by the Puerto Rico Louis Stokes Alliance for Minority Participation Program, and one by the American Society for Microbiology Undergraduate Research Capstone Program. Furthermore, she had the privilege to present in a series of regional and national meetings, in which she was awarded Second Place Oral Presentation by the Puerto Rico Society for Mycologists. After graduation, she worked as a postbaccalaureate student at Michigan State University in the Department of Pharmacology and Toxicology studying the role of AMPA receptors in methylmercury-induced toxicity in hiPSC-MN. She is currently pursuing her graduate studies at the University of Arizona in the Arizona Biological and Biomedical Sciences Program specializing in Plant Pathology, where she was currently awarded an NSF-GRFP fellowship. 


\section{PRESS SUMMARY}

For years, rice has been one of the most important crops in the world. Approximately $75 \%$ of the human population includes it in their daily diet because of its nutritional value. However, these crops can be attacked by various pathogens, leading to production losses, and adverse health effects in animals and humans. One of the most important groups of pathogens and major crop contaminants are fungi. The study presented herein compares the surface and endophytic fungal microflora present on medium fortified white and brown rice commercially sold in Puerto Rico. The goal of this research is to identify whether commercially sold rice in Puerto Rico are contaminated with fungal pathogens, and the difference in fungal incidence between medium fortified white vs. brown rice The identification and classification of these organisms is vital to elucidate their behavior and mechanisms of action in the environment, as well as designing new methods for prevention and control; therefore, decreasing crop contamination and human exposure. 


\title{
Characterization of Ambient Particulate Matter Sampled at an Active Sand Mine Facility in Northwestern Wisconsin
}

\author{
Julie Zhang, ${ }^{1,2}$ Callie Fischer, ${ }^{1}$ Joe Oster, ${ }^{1}$ Gabriel de Carvalho Chaves, ${ }^{1,3}$ Rachel Mooney, ${ }^{1}$ \& Patricia Cleary ${ }^{1 *}$ \\ ${ }^{1}$ Department of Chemistry, University of Wisconsin - Eau Claire, Eau Claire, WI \\ ${ }^{2}$ now at University of Wisconsin-Madison, Madison, WI \\ ${ }^{3}$ now at Pontifícia Universidade Católica de Minas Gerais, Brazil \\ Students: jzhang555@wisc.edu*,fischerca@uwec.edu,joester93@hotmail.com,gabrielcarch@gmail.com,mooney.rachel23@gmail.com \\ Mentor: clearypa@uwec.edu*
}

\begin{abstract}
Concern has arisen about levels of silica in ambient particles near sand mines in Northwestern Wisconsin. Airborne particles released from mining and processing activity may release respirable silica into the air, which can have adverse health effects on individuals exposed to significant quantities. In order to assess these levels of silica, this study developed a parallel analysis using an X-ray diffraction (XRD) and scanning electron microscopy/energy-dispersive X-ray spectroscopy (SEM-EDS) analysis to test particles in real air samples. Calibrations were constructed for the XRD analysis (following NIOSH Method 7500) with silica standards containing $10 \mu \mathrm{g}-500 \mu \mathrm{g}$ respirable silica on filter media with detection limits of 19-28 $\mu \mathrm{g}$. SEM-EDS methods incorporated identifying the geologic composition of particles using the elemental analysis. Real air samples were collected at a sand mining site using a cascade impactor. Filter substrates were pre-weighed and post-weighed to determine the total dry mass of particles sampled and XRD results show at maximum $16 \%$ of the mass can be attributed to crystalline silica in the samples. An SEM-EDS analysis to categorize the particles geologic classification using ratios of elements shows more than $70 \%$ of sampled particles are classified as potassium feldspars.
\end{abstract}

\section{KEYWORDS}

Particulate Matter; Sand Mining; Silica; Atmospheric Characterization; XRD; SEM-EDS; Fugitive Dust

\section{INTRODUCTION}

Airborne particulate matter is a highly variable and complex mixture of solid or liquid matter. Both natural and anthropogenic sources are responsible for the distribution of primary particulate matter in air. Particles with the potential to cause health effects are associated with its size, composition, and concentration in the air, where typical federal regulations identify hazards by particle size and concentration. ${ }^{1}$ Particulate matter composed of crystalline silica poses a health threat. ${ }^{2}$ Emission of silica-containing particulate matter may occur during sand mining in Wisconsin as sandstone is crushed and broken apart, releasing dust particles into the air. ${ }^{3}$ The sand mining industry has grown immensely in Wisconsin in the past years, from just 8 industrial sand facilities in 2008 to 129 in 2015, making Wisconsin the nation's leading sand producer due to the demand for specialized sand used in hydrofracking operations (referred to as frac sand mining). ${ }^{4}$ As the number of frac sand mining facilities in Wisconsin increased rapidly, concern has arisen on the correlation between the sand mining boom and the potential for increasing ambient particle concentrations which may contain respirable crystalline silica. Residents near the frac sand sites have concerns about the potential exposure to respirable crystalline silica in the air. With no federal or state guidelines or standards for respirable crystalline silica in ambient air, Minnesota Department of Health and California Office of Environmental Health Hazarded Assessments established an air quality chronic health-based value $(\mathrm{HBV})$ of $3.0 \mu \mathrm{g} / \mathrm{m}^{3}$ for respirable crystalline silica. ${ }^{5}$

Silica, silicon dioxide $\left(\mathrm{SiO}_{2}\right)$, is one of the most abundant compounds in Earth's crust being present in most of the rocks and soils. It is found with both amorphous microstructure and crystalline structure, and three principal forms: quartz, cristobalite, and tridymite. Crystalline silica particles, primarily quartz dust, are known to be a human carcinogen. ${ }^{6}$ Respirable crystalline silica is defined as silicon dioxide particulates of 4 micrometers or less in diameter $\left(\mathrm{PM}_{4}\right)$ by OSHA. ${ }^{7}$ Excess exposure to these particles can lead to the development of silicosis, airway diseases, pulmonary tuberculosis, chronic renal disease, and lung cancer. ${ }^{1} \mathrm{PM}_{10}$ silica particles can become airborne and be inhaled and enter the lungs. Particles of a diameter of $4 \mu \mathrm{m}$ or less pose a greater health hazard. Not only are these particles small enough to enter the lungs, but they can also travel into the alveolar regions of the lungs where they can cause irreparable damage. ${ }^{8}$ Alveolar macrophages engulf but fail to dissolve the particles, triggering an inflammatory response. ${ }^{9}$ The scar tissue left by this damage can, over time, result in the production of shortness of breath, poor gas exchange in the lungs, fatigue, and in extreme cases, respiratory failure.

The Occupational Safety and Health Administration established a Reference Exposure Limit (REL) for respirable crystalline silica at $50 \mu \mathrm{g} / \mathrm{m}^{3}$ averaged over an 8-hour working shift. ${ }^{10}$ The recommended analysis, NIOSH method 7500, ${ }^{7}$ uses XRD analysis to determine silica concentrations. This method aims to collect atmospheric particles with the use of polycarbonate filters and cyclones samplers. Samples are prepared by ashing the filters and depositing the resulting ash onto silver filters, which are then 
analyzed by XRD. NIOSH Method 7500 has been used by Sanderson et al. to evaluate respirable silica abundance near sand mines. ${ }^{2}$ Other studies that utilize the NIOSH method to measure respirable silica include Shiraki et al.'s study ${ }^{11}$ on silica exposure near a sand facility in central California, and Richards et al. studies ${ }^{12,13}$ at Aggregate-Producing-sources in California and at frac sand mining facilities in Wisconsin.

The sandstone material being mined in western Wisconsin consists mainly of well-rounded quartz grains (95\%), a majority in the $0.3-0.6 \mathrm{~mm}$ diameter range, ideal for use in hydraulic fracking operations ${ }^{14}$. The interstitial spaces consist of void spaces and cement made up of dolomite, hematite, authigenic potassium feldspar, illite, and authigenic quartz, based on a previous study on the Wowenoc and Jordan formations. ${ }^{15}$ The components of the cement pose no added health concern for their composition in particulate matter. Due to the history of crystalline silica particle emissions from sand mining, this study is aimed at investigating the amount of quartz found in ambient particles emitted at these mines.

Two studies have investigated particles in areas near to frac sand mines in Wisconsin. Walters et al. investigated $\mathrm{PM}_{2.5}$ airborne particulates near frac sand operations using an SKC DPS sampler to determine $\mathrm{PM}_{2.5}$ concentrations. ${ }^{3}$ Authors of this study assumed that all the $\mathrm{PM}_{2.5}$ particles counted were silica, concluding that there is an increase of ambient $\mathrm{PM}_{2.5}$ levels as a result of increasing frac sand mining. On the other hand, another study by John Richards at EOG facilities monitored air emissions for crystalline silica at $\mathrm{PM}_{4}$ and collected 657 average daily measurements from the fence lines of sand producing facilities in Wisconsin. ${ }^{13}$ The study described measurements of local $\mathrm{PM}_{2.5}$ monitoring stations as representing regional fine particle loading, and the results showed there is little influence by nearby sand mine operations. NIOSH Method 7500 was followed, sample values below the limit of quantification were treated as zeros, XRD results showed the average long-term ambient $\mathrm{PM}_{4}$ crystalline silica concentrations ranged between 0.05 and $0.45 \mu \mathrm{g} / \mathrm{m}^{3}$, from $5 \%$ to $20 \%$ of the Minnesota Department of Health's and California Office of Environmental Health Hazarded Assessments' exposure level of $3.0 \mu \mathrm{g} / \mathrm{m}^{3}$. However, this study only measured the concentration of crystalline silica at $\mathrm{PM}_{4}$, and the composition of the particles that were not silica remained unresolved.

One way to solve the discrepancy between these two studies is to conduct some elemental analysis on the sampled particles using SEM-EDS. This strategy has been employed successfully by many groups to develop parameters for principal component analysis and source apportionment. ${ }^{16-18}$ Moreno et al. ${ }^{19}$ used scanning electron microscopy-energy dispersive spectroscopy in order to characterize the geology of ambient particles. Many others have used multi-instrumentation approaches to characterize ambient particles and their sources better. ${ }^{20,21}$

To have a better understanding of both the levels of crystalline silica and elemental composition of particles in the air, this study quantitatively and qualitatively measured respirable particles near frac sand mining sites in Wisconsin. Because of the toxicity of respirable crystalline silica, the analysis focuses on the identification of quartz with respect to other materials in the particles. Scanning electron microscopy-energy dispersive spectroscopy (SEM-EDS) and X-ray diffraction (XRD), were used to directly test for crystalline silica and other geologic parent material using elemental analysis. Samples for XRD analysis were prepared based on a modified version of NIOSH method 7500 .

\section{METHODS AND PROCEDURES}

\section{Sample Collection}

Samples were collected using two Sioutas Personal Cascade Impactors, each composed of four stages, used to separate particulate matter based on size. Using the Leland Legacy Sample Pumps at a flow rate of $9 \mathrm{~L} / \mathrm{min}$ for 24 hours, filters were sampled at the Fairmont Santrol mine in Menomonie, WI next to the "Grizzly," a rock crusher with no fugitive dust control measures in an open area. Typically the machine was operated in either 2 or 3 shifts (16 to 24 hours a day). The cascade impactors were each loaded with four pre-weighed $25 \mathrm{~mm}$ diameter $2.0 \mu \mathrm{m}$ pore size filters made of polycarbonate (for XRD analysis) or PTFE (for SEMEDS analysis). Airborne particles were separated into four size ranges: $>2.5 \mu \mathrm{m}, 1.0-2.5 \mu \mathrm{m}, 0.50-1.0 \mu \mathrm{m}$, and $0.25-0.50 \mu \mathrm{m}$, corresponding to stages A, B, C, and D respectively. An identical field blank filter was used as a control for comparison. Each set of samples was collected for 24 hours. Pre- and post-weighing of desiccated filters to the nearest $0.01 \mathrm{mg}$ was performed to determine deposited particle mass. The weighing process was performed a minimum of three times until a variability of less than $0.1 \%$ was achieved.

\begin{tabular}{|l|c|c|c|}
\hline Sampling Date & SEM analysis & XRD analysis & Gravimetric \\
\hline $6 / 25 / 15$ & $\mathrm{X}$ & & \\
\hline $7 / 27 / 15$ & $\mathrm{X}$ & $\mathrm{X}$ & \\
\hline $9 / 24 / 15$ & $\mathrm{X}$ & $\mathrm{X}$ & $\mathrm{X}$ \\
\hline $11 / 15$ & & $\mathrm{X}$ & $\mathrm{X}$ \\
\hline
\end{tabular}

Table 1: Overall sampling at Menomonie site with two particle samplers equipped with filters for either SEM or XRD analysis. Gravimetric analysis was conducted on filters used for XRD analysis that were pre-weighed before sampling. 


\section{XRD Analysis}

The quartz component of the particles has significant health implications, so the XRD analysis focused exclusively on the identifying the amount of crystalline silica collected. Sample preparation for analysis by X-ray diffraction was performed using a modified version of NIOSH Method 7500. Polycarbonate and silica standard filters were ashed in a muffle furnace at $500^{\circ} \mathrm{C}$ for 2 hours. The ash was then suspended in 2-propanol, and the suspension was subjected to an ultrasonic bath for 30 minutes to break up agglomerated particles. Particles were deposited onto a silver filter using a vacuum filtration apparatus. The filters were heatfixed to a glass slide using Parlodion solution and mounted on the XRD (Bruker D8 Discover).

A calibration curve for the XRD analysis method was constructed using $10 \mu \mathrm{g}, 20 \mu \mathrm{g}, 50 \mu \mathrm{g}, 100 \mu \mathrm{g}, 250 \mu \mathrm{g}$ and $500 \mu \mathrm{g}$ silica calibration standard filters from NIST's Standard Reference Material division. Filters were subjected to the sample preparation method and analyzed by XRD. Peak areas were measured versus quartz masses to derive a linear equation. The detection limit, DL, was calculated as:

$$
\mathrm{DL}=3 \mathrm{~s}_{\mathrm{x}} / \mathrm{m}
$$

\section{Equation 1.}

where $s_{x}$ is the standard deviation of the intercept, and $m$ is the slope of the linear calibration curve generated. Two calibrations were made on July 25, 2015, and June 6, 2016. The equation derived from the standard calibration curve was used to solve for quartz mass in the three samples from the field using the peak areas obtained from the sample XRD spectra.

Full XRD rotation was used to ensure use of continuous angles of a sample to help eliminate random errors that may occur when repeatedly testing multiple fixed angles. Peak areas were measured from the peak maximum at $26.66^{\circ}(2 \theta)$ to $\pm 4 *$ width to calculate the tails range for each peak (Figure 1). Due to the time-intensive nature of the XRD scans ( $\sim 5$ hours each) and the small sample material amounts, the XRD was only used to quantify quartz.

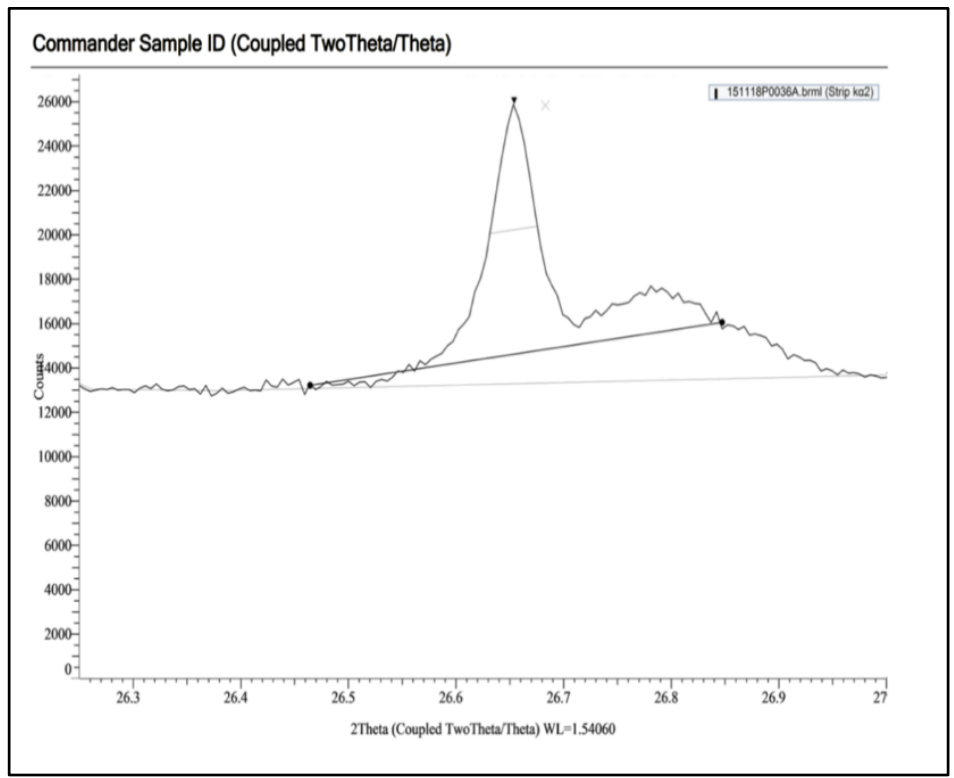

Figure 1. Area under the primary peak of quartz at $26.66^{\circ} 2 \theta$ was calculated using XRD

\section{SEM-EDS Analysis}

Elemental characterization of the particles was done using a scanning electron microscope. Particles from the sample were transferred onto carbon tape which was placed on an aluminum stub. The particles were analyzed using scanning electron microscopy (Hitachi S-3400N Variable Pressure SEM) coupled with energy dispersive spectroscopy (EDS with 1-micron resolution). The microscope working distance was $10 \mathrm{~mm}$ with an accelerating voltage of $15 \mathrm{kV}$ and had an acquisition of $60 \mathrm{~s}$ live time. The X-ray point-and-shoot and linescan methods were used to run the analysis. The point and shoot method focused on a specific point on a particle, providing an elemental analysis of a particular spot (See Figure 2). The sample was broken up into three zones and six sections. A random number generator was used to select one of these sections to analyze, and this process was repeated 6 times per filter. The points analyzed formed a grid of six points, three on top and three below per section totaling $\mathrm{N}=36$ for each filter stage (if sufficient particles were available). The point-and-shoot analysis can only perform this analysis on an area down to $0.5 \mu \mathrm{m}$ in diameter, so this method was only used for elemental analysis of the largest particulate sample, stage A.

The linescan method was used to analyze the other three stages, B-D on 9/24/15. Rather than analyzing individual points, the linescan method analyzes 100 points along a line to produce a spectrum, allowing a larger sample of particles to be 
analyzed per each section. Each filter sample produced 1200 data points. In the linescan method, the sample was broken up into five zones and each zone into six sections. Like the point-and-shoot method, one of the six sections was selected for analysis using a random number generator. Four linescans were run in the shape of a pound sign to analyze multiple particles in the sample. This process was repeated for each of the five zones. The data collected was categorized into different geological classes, which was modified from the analysis outlined in Moreno et al. ${ }^{19}$ using determined atom percent (Table 2).

\begin{tabular}{|c|c|c|c|c|c|c|}
\hline $\begin{array}{l}\text { Composition } \\
\text { label }\end{array}$ & $\begin{array}{l}\text { Identifying } \\
\text { factor }\end{array}$ & $\begin{array}{l}\mathrm{Al} \#= \\
\mathrm{Al} / \mathrm{SUM}\end{array}$ & $\begin{array}{l}\text { Mg\#= } \\
\text { Mg/SUM }\end{array}$ & $\begin{array}{l}(\mathrm{Mg}+\mathrm{Fe}) \#= \\
(\mathrm{Mg}+\mathrm{Fe}) / \mathrm{SUM}\end{array}$ & $\overline{\mathrm{Al} / \mathrm{Si}}$ & $\begin{array}{l}\mathrm{Si} \#= \\
\mathrm{Si} / \mathrm{SUM}\end{array}$ \\
\hline Other & $\begin{array}{l}\text { Large other } \\
\text { elemental } \\
\text { component }\end{array}$ & & & & & \\
\hline Illite & $\begin{array}{c}\mathrm{Mg}+\mathrm{Fe}>5 \% \\
\text { SUM }\end{array}$ & & & & & \\
\hline Hematite & $\begin{array}{c}\mathrm{Fe}>5 \% \\
\text { SUM }\end{array}$ & & & & & \\
\hline K-Feldspar & & $\mathrm{Al} \#<25$ & $\mathrm{Mg} \#<25$ & $(\mathrm{Mg}+\mathrm{Fe}) \#<25$ & $\mathrm{Al} / \mathrm{Si}>0.2$ & $\mathrm{Si} \#>50$ \\
\hline Quartz & $\begin{array}{c}\text { Si dominant } \\
\text { (SUM - Si< } \\
10 \%)\end{array}$ & $\mathrm{Al} \#<25$ & $\mathrm{Mg} \#<25$ & $(\mathrm{Mg}+\mathrm{Fe}) \#<25$ & $\mathrm{Al} / \mathrm{Si}<0.2$ & $\mathrm{Si} \#>50$ \\
\hline Diesel & $\begin{array}{l}\text { Sulfur atom } \\
\%>5 \%\end{array}$ & & & & & \\
\hline
\end{tabular}

Table 2. Classification scheme for SEM-EDS analysis. The inputs are the atom percent of $\mathrm{Al}, \mathrm{Mg}$, $\mathrm{Si}$, and $\mathrm{Fe}$, where $\mathrm{SUM}=(\mathrm{Al}+\mathrm{Mg}+\mathrm{Fe}+\mathrm{Si}+\mathrm{K})$.

a)

Filter-C(1)

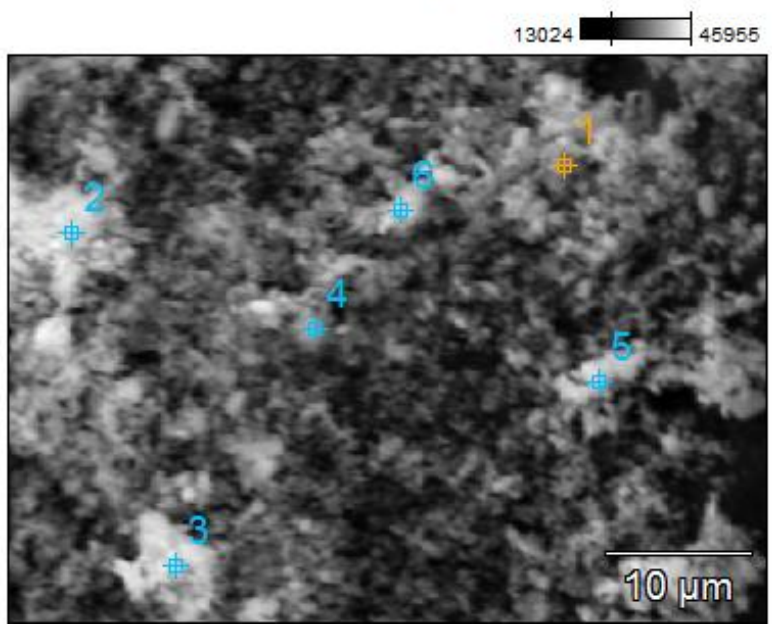

Full scale counts: 6048

Filter-C(1)_pt1

b)

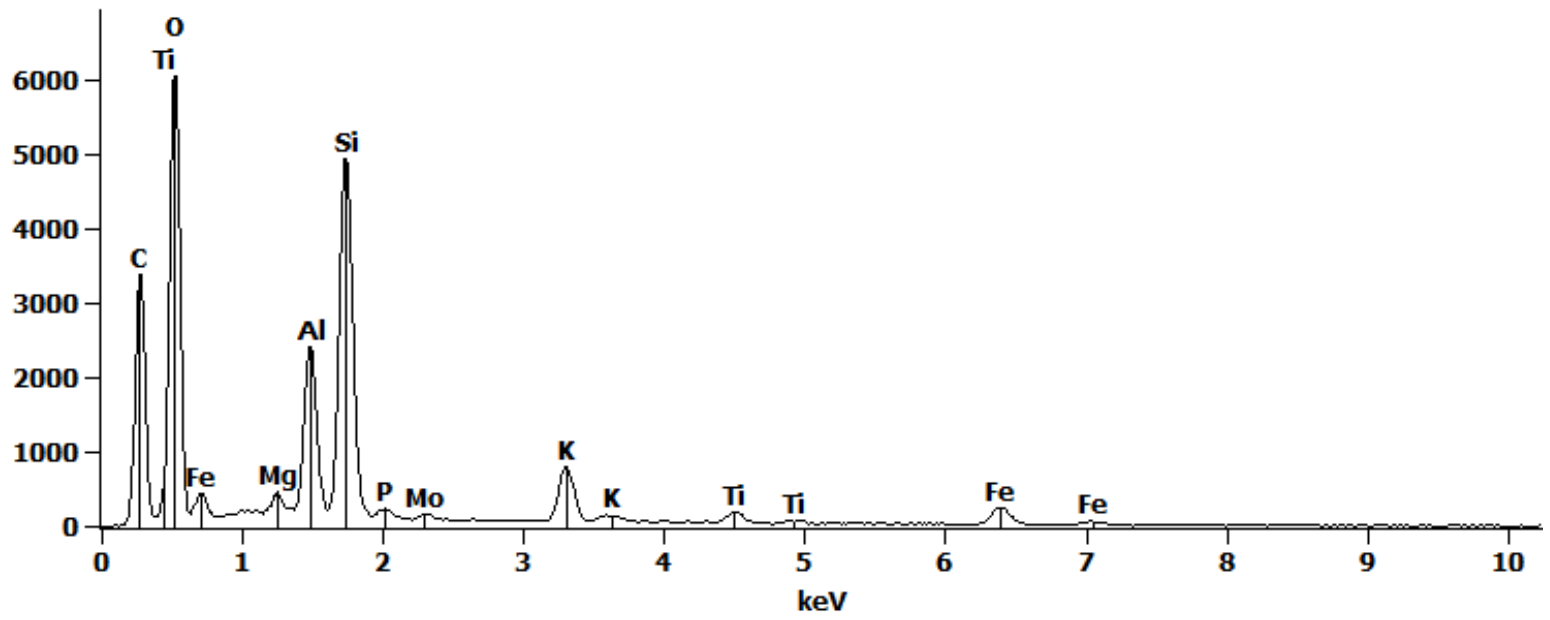

Figure 2. Example SEM image (a) of Stage C sample from 7/27/17 with EDS spectrum b) from Point 1. 


\section{RESULTS}

Four 24-hour samples were analyzed over the summer and fall of 2015, but not all analysis types were able to be conducted for all sampling days (Table 1). A calibration graph for XRD was made on June 6, 2016 gave a detection limit (DL) of 19 $\mu$ g, with y $=$ $0.00713( \pm 0.00018) \times-0.007$ ( \pm 0.044$)$. Particle mass deposited on the sample filters in the $2.5-10 \mu$ diameter group range from 250 to $1300 \mu \mathrm{g}$. Silica peak areas on the XRD were relatively small, requiring long time-averaged scans of 4-5 hours, with estimate quartz mass between 4-63 $\mu \mathrm{g}$. The nearest in time calibration curve was applied to each set of samples to process the data. Many samples were below the XRD detection limit for quartz. When above the detection limit, the percent quartz of the particle mass deposited on the filters was 16\%. Table 3 shows reported results for XRD analysis and gravimetric analysis. The particle concentration in the air was calculated from the particle mass, the 24-hour sampling period and the flow rate of the pump used with the cascade impactor (9 Liters per minute). The quartz concentration in the air was calculated similarly but starting with the calculated quartz mass from the XRD analysis.

Results from $7 / 27 / 2015$ had a detection limit of $28 \mu \mathrm{g}$. The decrease in silica net peak areas indicates smaller particle size and fewer particles collected on the filters. The calculated quartz mass from the particle size range of 2.5-10 $\mu \mathrm{m}$ was $63 \mu \mathrm{g}$. Preweights of filters were not measured. Therefore, overall particle mass deposited on filter and quartz percent on filters were unable to be obtained. Calculated quartz concentration in the air was $4.9 \mu \mathrm{g} / \mathrm{m}^{3}$ for particle size $2.5-10 \mu \mathrm{m}$, and $2.2 \mu \mathrm{g} / \mathrm{m}^{3}$ for particle size $1.0-2.5 \mu \mathrm{m}$. Results from Sept. 24, 2015, had a detection limit of $28 \mu \mathrm{g}$. Quartz mass from the particle size range of 2.5-10 $\mu \mathrm{m}$ is $39 \mu \mathrm{g}$, about $16 \%$ of the total particles collected on the filters. The calculated concentration of quartz in the air was $2 \mu \mathrm{g} / \mathrm{m}^{3}$. Results from $11 / 2015$ had a detection limit of $19 \mu \mathrm{g}$. Estimate quartz masses were all below the detection limit. It is noted that there were significant amounts of particles deposited on filters, ranging from $1330 \mu \mathrm{g}$ for particle size $2.5-10 \mu \mathrm{m}$, to $330 \mu \mathrm{g}$ for particle size $0.25-0.5 \mu \mathrm{m}$.

\begin{tabular}{|c|c|c|c|c|c|c|}
\hline $\begin{array}{l}\text { Cascade } \\
\text { Impactor Stages } \\
\text { (particle } \\
\text { aerodynamic } \\
\text { diameters) }\end{array}$ & $\begin{array}{l}\text { XRD } \\
\text { quartz } \\
\text { peak net } \\
\text { area } \\
\text { (counts } \times \\
\text { degrees) } \\
\end{array}$ & $\begin{array}{l}\text { Calculated } \\
\text { quartz mass } \\
(\mu \mathrm{g})\end{array}$ & $\begin{array}{l}\text { Particle } \\
\text { mass } \\
\text { deposited on } \\
\text { filters }(\mu \mathrm{g})\end{array}$ & $\begin{array}{l}\text { \% Quartz } \\
\text { on Filters }\end{array}$ & $\begin{array}{l}\text { Particle } \\
\text { concentration } \\
\text { in the air } \\
\left(\mu \mathrm{g} / \mathrm{m}^{3}\right)\end{array}$ & $\begin{array}{l}\text { Quartz } \\
\text { concentration } \\
\text { in the air } \\
\left(\mu \mathrm{g} / \mathrm{m}^{3}\right)\end{array}$ \\
\hline \multicolumn{7}{|c|}{ Sample Date: 07/27/2015 } \\
\hline $\mathrm{A}(2.5-10 \mu \mathrm{m})$ & 0.3736 & 63 & - & - & - & 4.9 \\
\hline B (1.0-2.5 $\mu \mathrm{m})$ & 0.1007 & 28 & - & - & - & 2.2 \\
\hline $\mathrm{C}(0.50-1.0 \mu \mathrm{m})$ & 0.08242 & $<\mathrm{DL}$ & - & - & - & - \\
\hline $\mathrm{D}(0.25-0.5 \mu \mathrm{m})$ & 0.03213 & $<\mathrm{DL}$ & - & - & - & - \\
\hline Blank & 0.01325 & $<\mathrm{DL}$ & - & - & - & - \\
\hline \multicolumn{7}{|c|}{ Sample Date: 09/24/2015 } \\
\hline A $(2.5-10 \mu \mathrm{m})$ & 0.1951 & 39 & 250 & $16 \%$ & 19 & 3.0 \\
\hline B $(1.0-2.5 \mu \mathrm{m})$ & 0.04110 & $<\mathrm{DL}$ & 70 & - & 5 & - \\
\hline $\mathrm{C}(0.50-1.0 \mu \mathrm{m})$ & - & - & - & - & - & - \\
\hline $\mathrm{D}(0.25-0.5 \mu \mathrm{m})$ & 0.002000 & $<\mathrm{DL}$ & 50 & - & 4 & - \\
\hline Blank & - & - & - & - & - & - \\
\hline \multicolumn{7}{|c|}{ Sample Date: 11/2015 } \\
\hline $\mathrm{A}(2.5-10 \mu \mathrm{m})$ & 0.02115 & $<\mathrm{DL}$ & 1330 & - & 103 & - \\
\hline B (1.0-2.5 $\mu \mathrm{m})$ & 0.01126 & $<\mathrm{DL}$ & 560 & - & 43 & - \\
\hline $\mathrm{C}(0.50-1.0 \mu \mathrm{m})$ & 0.008146 & $<\mathrm{DL}$ & 330 & - & 25 & - \\
\hline $\mathrm{D}(0.25-0.5 \mu \mathrm{m})$ & 0.006028 & $<\mathrm{DL}$ & 330 & - & 25 & - \\
\hline Blank & - & - & - & - & - & - \\
\hline
\end{tabular}

Table 3. Particle mass, XRD Data and Calculated Values for polycarbonate filters.

SEM-EDS analysis determined the particle composition of the samples. The main elements found in all samples were, in decreasing order, oxygen (53.8\%), silicon (23.2\%), aluminum (9.3\%), potassium (5.8\%), iron (3.4\%) and magnesium (1.4\%). The sum of atom percent of all elements excluding carbon was from 58-99\% for all samples. Carbon was excluded from the analysis due to interference from the carbon tape used in the SEM analysis (blank scans on the SEM-EDS show carbon as the main 
element). Samples with the highest amount of carbon were samples with fewer particles transferred to the carbon tape, consistent with the assumption that the carbon signal is background.

Particles were placed into five categories: potassium feldspar (abbreviated as kspar), quartz, hematite, illite, diesel and other based on their elemental composition. The identifying characteristics sorted based atom percent of elements, was established from both SEM-EDS analysis of thin-film sections of the Jordan formation with geologic identification from previous work, ${ }^{15}$ samples of pure quartz, calcite, hematite, diesel particles and modifications of the analysis established in Moreno $\mathrm{et}$ al. ${ }^{19}$

(See Table 2). For all sampling dates, the majority of sampled particles were classified as potassium feldspars (see Table 4), which could also be considered kaolinite clays based on the depositional history of the formation. ${ }^{15}$ The composition varies slightly for cements that have some characteristics of hematite (high iron content). While we classify these as hematite, the identifying signature is being iron-rich based on the other elements observed.

\begin{tabular}{|c|c|c|c|c|c|}
\hline & Kspar & Hematite & Illite & Quartz & Other \\
\hline \multicolumn{6}{|l|}{$6 / 25 / 15$} \\
\hline $\mathrm{A}(2.5-10 \mu \mathrm{m})$ & $94.44 \%$ & 0.00 & 0.00 & 5.56 & 0.00 \\
\hline B $(1.0-2.5 \mu \mathrm{m})$ & 86.67 & 5.00 & 0.00 & 1.67 & 6.67 \\
\hline $\mathrm{C}(0.50-1.0 \mu \mathrm{m})$ & 90.00 & 6.67 & 0.00 & 0.00 & 3.33 \\
\hline $\mathrm{D}(0.25-0.5 \mu \mathrm{m})$ & 80.00 & 13.33 & 0.00 & 3.33 & 3.33 \\
\hline \multicolumn{6}{|l|}{$7 / 27 / 15$} \\
\hline $\mathrm{A}(2.5-10 \mu \mathrm{m})$ & 70.00 & 23.33 & 0.00 & 0.00 & 6.67 \\
\hline B $(1.0-2.5 \mu \mathrm{m})$ & 53.33 & 43.33 & 0.00 & 3.33 & 0.00 \\
\hline $\mathrm{C}(0.50-1.0 \mu \mathrm{m})$ & 86.67 & 10.00 & 0.00 & 0.00 & 0.00 \\
\hline $\mathrm{D}(0.25-0.5 \mu \mathrm{m})$ & 76.67 & 23.33 & 0.00 & 0.00 & 0.00 \\
\hline \multicolumn{6}{|l|}{$9 / 24 / 15$} \\
\hline A $(2.5-10 \mu \mathrm{m})$ & 61.11 & 33.33 & 0.00 & 2.78 & 2.78 \\
\hline B $(1.0-2.5 \mu \mathrm{m})$ & 95.20 & 3.65 & 0.09 & 0.00 & 1.07 \\
\hline $\mathrm{C}(0.50-1.0 \mu \mathrm{m})$ & 96.85 & 2.04 & 0.00 & 0.00 & 1.11 \\
\hline $\mathrm{D}(0.25-0.5 \mu \mathrm{m})$ & 49.94 & 49.94 & 0.00 & 0.00 & 0.13 \\
\hline
\end{tabular}

Table 4 Geologic composition of Teflon filter set sampled on 6/25/2015, 7/27/15, and 9/27/15 using SEM-EDS elemental analysis.

The largest particle size (Stage A) typically has more identifiable different particle categories, perhaps due to the more precise point-and-shoot sampling technique. However, it should be noted that for most of the particles sampled, some component can be attributed to quartz for almost all filter stages, but not necessarily uniformly. Figure 3 shows the overall average composition of all samples, of which $2.5 \%$ are classified as quartz using SEM-EDS. The percent of quartz particles in each filter stage was between 0.00 and $7.41 \%$ of the total particles. The ambient particles were mainly potassium feldspars (78.4\%), secondarily hematite $(17.8 \%)$, with small amounts of quartz $(2.1 \%)$. The "other" category corresponds to particles that were unidentifiable, some of which contained large amounts of phosphorus, and trace amounts of transition metals such as titanium, molybdenum, and scandium.

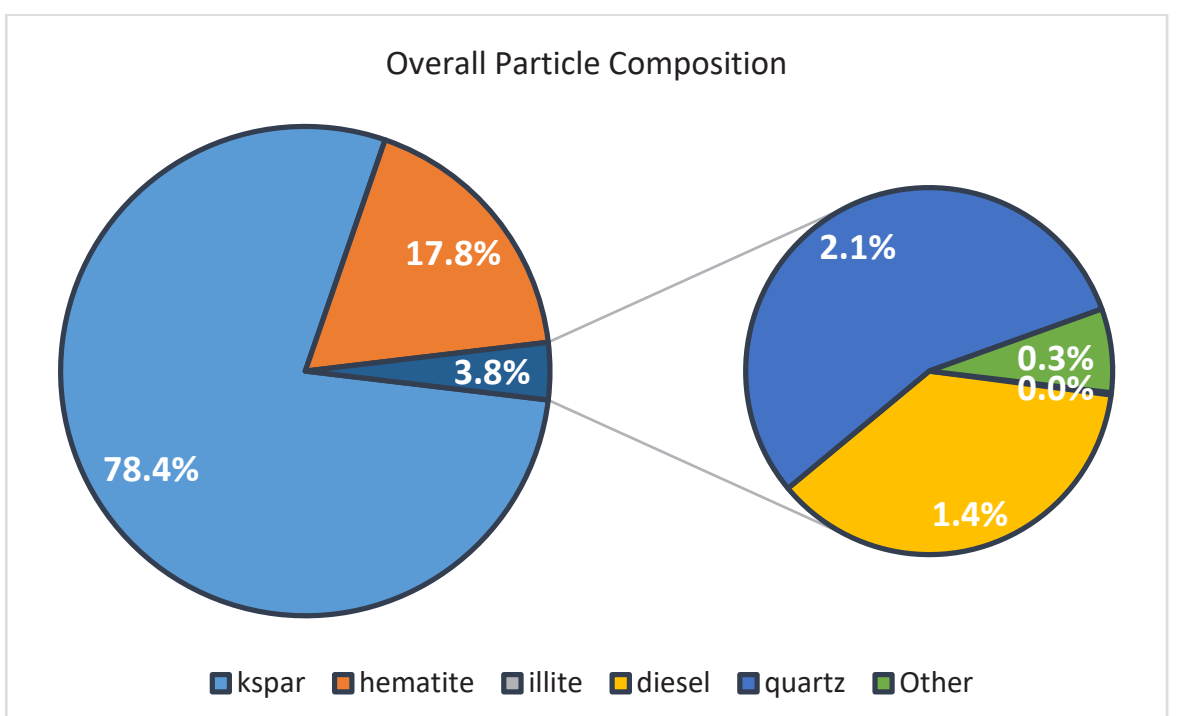

Figure 3. Pie chart of average results from three collection dates: June 25, 2015; July 27, 2015; September 24, 2015. The data show the percentage of results categorized by five subcategories of relatively 
When broken down into the filter stages (Figure 4) for all sampling days, the largest (A) and smallest (D) particle sizes also have more composition attributed to other classes besides potassium feldspar. We note the limitations of the SEM to target individual particles of smaller size on stages B-D. In these smaller stages, each data point analyzed corresponds to elemental analysis of a cluster of particles both under and around the specific point. Since a high percentage of the particles sampled are likely to be feldspars, they would mask the signal given off by different particles.

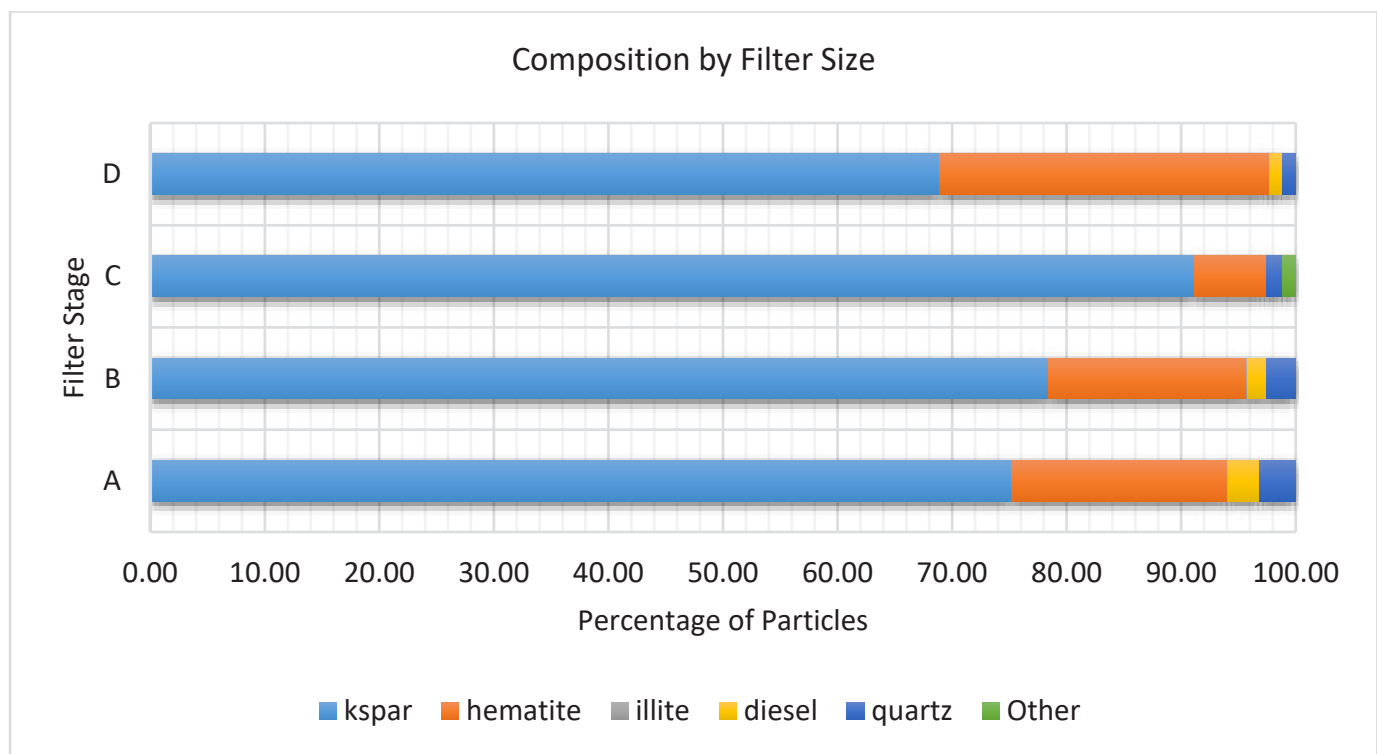

Figure 4. Average SEM-EDS results from three collection dates: 6/25/15; 7/27/15; 11/15. The data shows the distribution of the four subcategories of clays, and quartz, by filter stages.

\section{DISCUSSION}

Quartz makes up a small portion of the sampled particles emitted near a rock crusher in the sand mine, as determined by both the SEM-EDS analysis and the XRD analysis. The post-sampling weights in Table 3 show that there can be large amounts of particles deposited onto the filters, but that when those same filters are analyzed for quartz by XRD, it was not found above the experimental detection limits. The SEM-EDS analysis corresponds well to this showing that a majority of particles (over 75\%) are classified as potassium feldspars. The only data from the XRD that quantified quartz at $16 \%$ of particle mass was on a date where the simultaneously sampled particles used in the SEM-EDS analysis only show roughly $6 \%$ of the largest particle stage was quartz. If we assume all Stage A particles had 6\% quartz, on 9/24/15 that would correspond to $15 \mu \mathrm{g}$ quartz, below the XRD detection limit, but on $11 / 15$ this would correspond to $80 \mu \mathrm{g}$, which would have been above the detection limit. We consider these in rough agreement as the SEM-EDS analysis is not by mass and is semi-qualitative because not all particles are analyzed, and the analysis classifies by atom $\%$ instead of measurement by mass weight. The conclusions that the majority of particles sampled at the rock crusher in a sand mine also aligns with geological thin films studies of the sandstone formation, which assert that the large quartz spheres in the rock formation have a large diameter, much larger than would be airborne naturally, but the sandstone formation is held together by cements such as calcite, hematite and potassium feldspars. ${ }^{22}$ These geological cements are smaller grain sizes within the rock formation and take up room in between the quartz spheres, and therefore are already closer to a size to become readily airborne $(10 \mu \mathrm{m}$ in diameter or less).

Weaknesses in this analysis are the exact identification of geologic materials from the SEM-EDS analysis. Our main goal was to identify quartz and be able to distinguish between it and other materials. Moreno et al. ${ }^{19}$ applied an SEM-EDS technique to classify different types of silicates into different 5 different categories. These classification techniques were adopted and modified in this study because of the similar distribution of elements found in this study, which we used mainly to identify quartz (Moreno’s Si-Felsic) and potassium feldspars (Moreno's AlSi-Felsic). The particles that we identify as hematite could be hematite combined with a feldspar, or could be contaminated with iron from mechanical wear of the rock crusher. There are few particles in our study containing magnesium and iron. We interpreted a large sulfur component to be diesel particulates, based on our analysis of diesel particles which show high carbon and sulfur peaks. The "other" category corresponds to particles that were unidentifiable, some of which contained large amounts of phosphorus, and trace amounts of transition metals such as titanium, molybdenum, and scandium. Even if the identification of non-quartz particles has higher uncertainty, our results with respect to the quartz content remain consistent with both the XRD and SEM-EDS analysis. At the ultra-fine stage, $0.25-0.50 \mu \mathrm{m}$, the most deeply respirable fraction of particles, the sample is primarily composed of feldspars and secondarily hematite, or iron-enhanced material (Figure 4). The percent of quartz particles in each filter stage was between 0.00 and $6.7 \%$ of the total particles. 
SEM-EDS data show the particle sample consisted mostly of geologic materials instead of anthropogenic sources. The analyses obtained revealed that both fine and coarse ambient particles sampled were consistently silicates containing both aluminum and potassium, which leads us to assume they are potassium feldspars, although the origin of the feldspars could have come from previous weathering and deposition into the sandstone formation. This is to be expected because quartz and feldspar are the most common minerals in the Earth's crust. Feldspars and subsilic minerals are the most common parent material of aluminum. ${ }^{23}$ This allows the conclusion that the weathering of feldspars resulted in clay materials present in the cement in the sandstone. In the samples collected, large amounts of the airborne feldspar are observed, which can be explained by the geology of sandstone. Odom ${ }^{14,24}$ discovered that fine-grained sandstone contained potassium feldspars. The cement is most likely represented by the feldspar category, specifically potassium feldspar, which holds the silica sand particles together. When the sandstone is crushed and fragmented at the sand-processing facility, the cement is destroyed, releasing an abundance of particulate feldspar into the air. Other cements that have been identified in these sandstone formations are calcite, quartz, hematite, goethite and silcrete. ${ }^{22}$ Our results do not indicate a significant amount of dolomite, illite or calcite in the observed particles. Although there is a high silica content in the geological features of sandstone, small amounts of quartz particles are present in the airborne particle samples. In the geological formation most desirable for frac sand mining, the quartz grains are fine-to-course, meaning the spherical quartz grains in the formation have diameters ranging from 100-500 $\mu \mathrm{m}$, larger by an order of magnitude from airborne particles. Because the mining process is to collect the silica sand particles, the processes are designed to abrade off the cement in the formation. Our results are consistent with the mechanical rock crushing producing smaller airborne particles consisting of geologic material found in the cement, rather than of quartz.

Observed differences between percent silica found on filters analyzed by XRD methods versus filters analyzed by SEM methods led to an investigation into possible interferences. Minerals commonly found in the mining sites were analyzed by XRD methods, and the locations of major peaks were compared to those found in a quartz sample. Calcite, kaolin, potassium feldspar, hematite, and muscovite were analyzed. Of these minerals, muscovite was the only one to present a significantly large peak in the same area as the major peak found in the quartz samples at about $2 \theta=26.8^{\circ}$ (Figure 5).

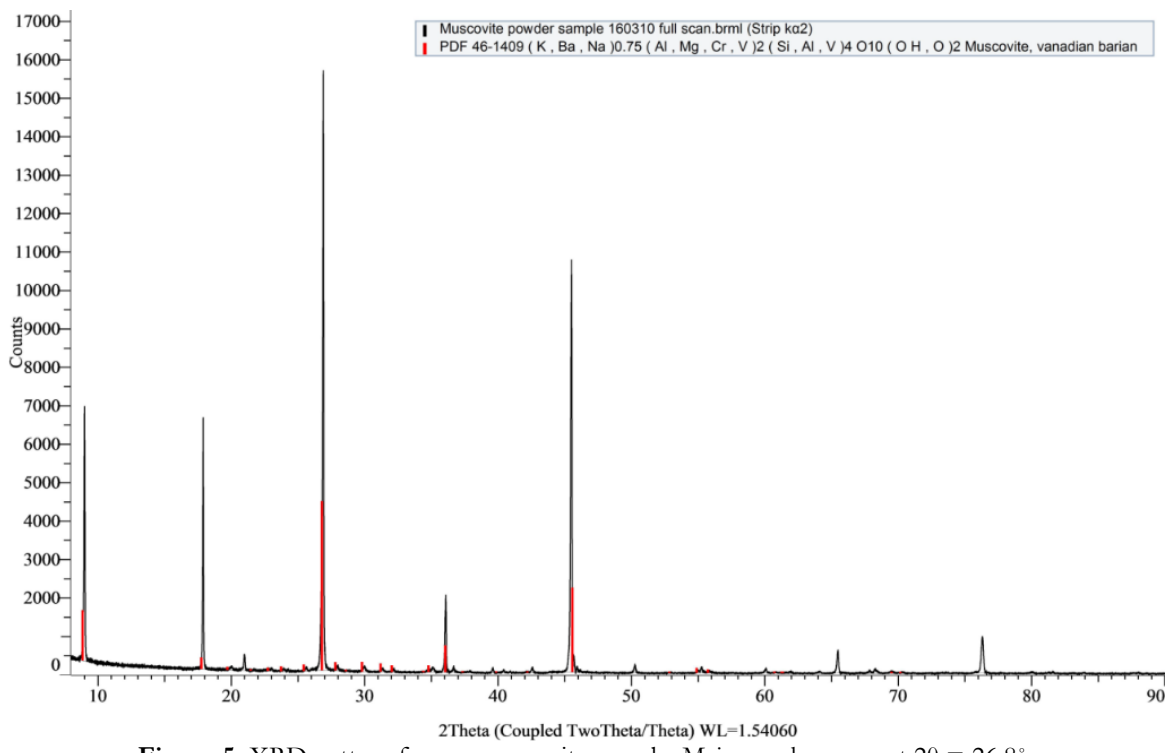

Figure 5. XRD pattern from a muscovite sample. Major peak occurs at $2 \theta=26.8^{\circ}$.

If some of the material on the filters contained muscovite, a high bias for quartz could have resulted for the XRD analysis, which could explain some higher values for the $\%$ quartz on the filters as measured by XRD. A systematic error of higher of higher quartz by the XRD analysis could come from the feature shoulder shown in Figure 1, where the baseline is not level. That said, even with a higher peak area at this diffraction angle, few measurements were quantifiable above the detection limit for a majority of the samples. Because the SEM-EDS analysis was more qualitative, misidentification quartz in the smaller particle sizes could lead to an underestimation of quartz. The consistent result from both XRD and SEM-EDS analysis is that quartz is a small component $(<20 \%)$ of particulate matter from mining a rock formation that is $95 \%$ quartz.

A future study could employ sampling at different locations at the sand mining facility. The sampling strategy presented here was focused on an area where dust control measures were not taken at a location of active rock crushing. The emissions from this site could be representative of some particle-emitting processes in the mine (for example, when dynamite is used on outcrops), but would be less representative of other possible sources of ambient particles (for example, during the processing, sorting and storage of sand) that could arise from abrasion of quartz sand. Therefore, this study is not comprehensive for all ambient particles emitted in a sand mine. 


\section{CONCLUSIONS}

The mass of particles typically collected on the filter samples during a 24-hour time period was about $2 \mathrm{mg}$, so a silica concentration of at least $32 \%$ would be needed to exceed the NIOSH Reference Exposure Level of $50 \mu \mathrm{g} / \mathrm{m}^{3} \mathrm{per} 8 \mathrm{hours}$. Silica levels determined from our analyses are well below, with the maximum observed at $1.6 \mu \mathrm{g} / \mathrm{m}^{3}$ per 8 hours. Overexposure to dust particles can be hazardous to health, and in the case of the sand mines, exposure to respirable silica is the most harmful, but we find little evidence of high levels of respirable silica. The X-ray diffraction analysis shows that levels of respirable crystalline silica at the rock crusher are well below the REL. Most sample quartz masses were below the detection limit, and the samples that did surpass this value were much lower than the REL. SEM-EDS analysis supported this same conclusion, finding that only a small percentage of the particles were identified as quartz. Rather, particles were composed primarily of the cement, specifically potassium feldspar, from the rock formation.

\section{ACKNOWLEDGMENTS}

The authors thank Brian Mahoney for his help in developing this project. This work was funded by the Office of Research and Sponsored Programs at UW-Eau Claire with the Student-Faculty Research Collaboration and the Summer Research Experiences for Undergraduates grants. Callie Fischer received support from UW-Eau Claire's Blugold Fellowship program. Gabriel de Carvalho Chaves was funded by the Brazil Scientific Mobility Program for Academic Training. The authors thank Fairmont Santrol for allowing us access to conduct air sampling at their facility.

\section{REFERENCES}

1. Davidson, C. I.; Phalen, R. F.; Solomon, P. A., (2005) Airborne particulate matter and human health: A review. Aerosol Sci Technol 39, 737-749.

2. Sanderson, W. T.; Steenland, K.; Deddens, J. A., (2000) Historical respirable quartz exposures of industrial sand workers: 1946-1996. Am J Ind Med 38, 389-398.

3. Walters, K.; Jacobson, J.; Kroening, Z.; Pierce, C., (2015) PM2.5 Airborne Particulates Near Frac Sand Operations. J Environ Health 78, 8-12.

4. Prengaman, K., Frac Sand Boom Creates Thousands of Jobs. WisconsinWatch.org: 2012. http:/ / wisconsinwatch.org/2012/08/sand-boom-creates-jobs/ (accessed Aug 2018)

5. Minnesota Department of Health (2013) Crystalline Silica Toxicological Summary. http:// www.health.state.mn.us/divs/eh/ hazardous/topics/silica/silicaguidance.html (accessed Aug 2018)

6. Borm, P. J. A.; Fowler, P.; Kirkland, D., (2018) An updated review of the genotoxicity of respirable crystalline silica. Part Fibre Toxicol 15, 17.

7. Ashley, K.; Fey O'Connor, P., (2016) NIOSH Manual of Analytical Methods. 5th ed.; National Institute for Occupational Safety and Health. https:// www.cdc.gov/niosh/docs/2003-154/chaps.html (accessed Aug 2018)

8. Castranova, V.; Porter, D.; Millecchia, L.; Ma, J. Y. C.; Hubbs, A. F.; Teass, A., (2002) Effect of inhaled crystalline silica in a rat model: Time course of pulmonary reactions. Mol Cell Biochem 234, 177-184.

9. Hamilton, R. F.; Thakur, S. A.; Holian, A., (2008) Silica binding and toxicity in alveolar macrophages. Free Radic Biol Med 44, 1246-1258.

10. O.S.H.A., Respirable Crystalline Silica Standard 1926.1153. https:// www.osha.gov/laws-regs/regulations/standardnumber/1926/1926.1153 (accessed Aug 2018)

11. Shiraki, R.; Holmen, B. A., (2002) Airborne respirable silica near a sand and gravel facility in central California: XRD and elemental analysis to distinguish source and background quartz. Environ Sci Technol 36, 4956-4961.

12. Richards, J. R.; Brozell, T. T.; Rea, C.; Boraston, G.; Hayden, J., (2009) PM4 Crystalline Silica Emission Factors and Ambient Concentrations at Aggregate-Producing-Sources in California. J Air Waste Manag Assoc 59, $1287-1295$.

13. Richards, J.; Brozell, T., (2015) Assessment of Community Exposure to Ambient Respirable Crystalline Silica near Frac Sand Processing Facilities. Atmosphere 6, 960-982.

14. Odom, I. E.; Wegrzyn, R. S.; Ostrom, M. E., (1978) Lithostratigraphy, petrology, and depositional environments of Jordan formation (Cambrian), Upper Mississippi Valley. Am Assoc Pet Geol Bull 62, 550-551.

15. Fliflet, R.; Poirier, J.; M.; Mahoney, J. B.; Syverson, K. (2016) Diagenetic History of Cambrian Sandstone Units in Western Wisconsin, GSA Abstr Prog: GSA Annual Meeting, Denver, CO.

16. Genga, A.; Baglivi, F.; Siciliano, M.; Siciliano, T.; Tepore, M.; Micocci, G.; Tortorella, C.; Aiello, D., (2012) SEM-EDS investigation on PM10 data collected in Central Italy: Principal Component Analysis and Hierarchical Cluster Analysis. Chem Cent J 6, 15.

17. Moroni, B.; Cappelletti, D.; Marmottini, F.; Scardazza, F.; Ferrero, L.; Bolzacchini, E., (2012) Integrated single particlebulk chemical approach for the characterization of local and long range sources of particulate pollutants. Atmos Environ 50 , 267-277.

18. Matassoni, L.; Pratesi, G.; Centioli, D.; Cadoni, F.; Lucarelli, F.; Nava, S.; Malesani, P., (2011) Saharan dust contribution 
to PM10, PM2.5 and PM1 in urban and suburban areas of Rome: a comparison between single-particle SEM-EDS analysis and whole-sample PIXE analysis. J Environ Monit 13, 732-742.

19. Moreno, T.; Gibbons, W.; Jones, T.; Richards, R., (2003) The geology of ambient aerosols: characterising urban and rural/coastal silicate PM10-2.5 and PM2.5 using high-volume cascade collection and scanning electron microscopy. Atmos Environ 37, 4265-4276.

20. Gonzalez, L. T.; Rodriguez, F. E. L.; Sanchez-Dominguez, M.; Leyva-Porras, C.; Silva-Vidaurri, L. G.; Acuna-Askar, K.; Kharisov, B. I.; Chiu, J. F. V.; Barbosa, J. M. A., (2016) Chemical and morphological characterization of TSP and PM2.5 by SEM-EDS, XPS and XRD collected in the metropolitan area of Monterrey, Mexico. Atmos Environ 143, 249-260.

21. Radulescu, C.; Stihi, C.; Iordache, S.; Dunea, D.; Dulama, I. D., (2017) Characterization of Urban Atmospheric PM2.5 by ATR-FTIR, ICP-MS and SEM-EDS Techniques. Rev Chim 68, 805-810.

22. Runkel, T.; Syverson, K.; Steenberg, J.; Bendernagel, M.; Merchison, T.; Bauer, A.; Kent, A.; Thompson, M.; Stauffer, T.; Brown, B. (2012) In Field Guidebook on the Silica Sand Resources of Western Wisconsin, Conference on the Silica Sand Resources of Minnesota and Wisconsin, Brooklyn Park, MN.

23. Keller, W. D., (1964) The Origin of High-Alumina Clay Minerals - A Review. In Clay and Clay Minerals: Vol. 2, $129-151$.

24. Odom, I. E., (1975) Feldspar-grain size relations in Cambrian arenites, Upper Mississippi Valley, J Sediment Petrol 45, 636650.

\section{ABOUT THE STUDENT AUTHORS:}

Julie Zhang is an undergraduate student studying chemistry at the University of Wisconsin-Madison. She transferred there from UW-Eau Claire in Spring 2017 and expects to graduate in Fall 2018. Callie Fischer graduated in Spring 2018 with a degree in biochemistry/molecular biology and plans to attend medical school. Joe Oster graduated from UW-Eau Claire in Spring 2015 with a B.S. in Chemistry. Gabriel de Carvalho Chaves was a Brazilian Exchange student under the Brazilian Scientific Mobility Program and is currently a production engineering major at Pontifícia Universidade Católica de Minas Gerais with a graduation date in 2019. Rachel Mooney will graduate from UW-Eau Claire in Spring of 2019 with a degree in chemistry.

\section{PRESS SUMMARY}

Concern has arisen about levels of silica in ambient particles near frac sand mines in Northwestern Wisconsin. Exposure to particles containing silica can cause well-known adverse health effects. This study developed a parallel analysis using an X-ray diffraction (XRD) and scanning electron microscopy/energy-dispersive X-ray spectroscopy (SEM-EDS) analysis to test particles for silica in real air samples. Real air samples were collected at a frac sand site using a cascade impactor. A standard method to analyze silica on filters, NIOSH Method 7500, was followed that can detect respirable silica down to of 10-44 $\mu \mathrm{g}$. The SEM-EDS used the elemental distribution found on the particles to assign parent geologic material. A majority $(>80 \%)$ of the particles studied were found to be made up of substances other than silica, as determined by both methods. 


\title{
Dextran Sulfate Based Gel Coating for Drug Eluting Balloon Angioplasty
}

\author{
Jenna Mosier*, Hannah Stealey, Kalifa Stringfield, Katie Webb, \& C. LaShan Simpson \\ Department of Agricultural and Biological Engineering, Mississippi State University, Mississippi State, MS \\ Students: jmosier243@gmail.com*, bannahstealey19@gmail.com,kalifastringfield@gmail.com, katiewebb1212@gmail.com \\ Mentor: clsimpson@abe.msstate.edu
}

\begin{abstract}
Vascular calcification, a consequence of cardiovascular disease, disrupts natural blood flow and can result in death. Common treatment efforts include various anti-inflammatory medications, balloon angioplasty, or stents, with little success in completely reversing calcification. The proposed design focuses on improving current drug-eluting stents by developing a dextran-sulfatebased gel drug delivery system loaded with receptor activator of nuclear kappa B-ligand (RANKL) to induce osteoclast differentiation. To ensure that the gel could adequately deliver RANKL, the gel was tested for its affinity for hydroxyapatite (HA), a critical component of calcification, and its ability to withstand shear. Infrared spectroscopy (IR) indicated binding to HA. Preliminary scanning electron microscopy and energy dispersive X-ray spectroscopy (SEM/EDS) results confirmed the presence of calcium on the gel after a one-hour soak in an HA mixture. Shear testing demonstrated that negligible protein, an average of $0.029 \pm 0.024 \mu \mathrm{g} / \mathrm{mL}$, was sheared off under flow conditions, indicating that the gel is stable for the duration of balloon delivery. These preliminary results indicate that a dextran-sulfate-based gel has the potential to serve as a therapeutic gel-coating to treat vascular calcification. Future experimentation will include a co-culture study to determine whether osteoclast progenitor cells will adequately proliferate and differentiate in the presence of the RANKL-loaded gel.
\end{abstract}

\section{KEYWORDS}

Angioplasty; Calcification; Cardiovascular; Dextran; Gel; Osteoclast; Stent; Vascular

\section{INTRODUCTION}

Cardiovascular disease is one of the most prevalent problems in the United States, causing one in three deaths annually. ${ }^{1}$ This condition can be manifested in various forms: heart disease, hypertension, diabetes, and many more. One particular consequence is vascular calcification. Smooth muscle cells lining the interior walls and in the medial layer of the artery are not terminally differentiated, meaning they can mature into different cell types. When calcium and phosphate levels increase in the bloodstream due to cardiovascular disease, they trigger a response in the tissue, causing arterial cells to differentiate into atypical phenotypes and deposit bone-like tissue. ${ }^{2}$ This active transformation of healthy tissue into diseased tissue along the lining of the artery causes the vasculature to stiffen and calcify, disrupting natural, laminar blood flow. ${ }^{3}$ The calcification prevents the arteries from dilating and constricting naturally and prevents blood flow through the lumen. The plaque buildup is living, inflamed tissue that continues to expand when aggravated. ${ }^{4}$

A current method used to treat vascular calcification is balloon angioplasty. During this procedure, a catheter is inserted through the femoral artery and fed to the site of calcification in the vasculature. A balloon is attached to the end of the catheter, typically one to five $\mathrm{mm}$ in diameter. ${ }^{5}$ Once in place, the balloon is inflated so that the force from deployment breaks up the calcification and clears the bloodstream. While this is temporarily successful, the recently disturbed tissue recruits more inflammatory molecules and restenosis, the return of plaque buildup, occurs. ${ }^{4}$ One way to counteract this issue is using an antiinflammatory agent to coat the balloon that can be released upon deployment. This design was first published in the 1980s, using the drug Paclitaxel as a counter-agent to inflammation. ${ }^{6}$ While it prolongs the time before restenosis occurs in the arteries; the use of Paclitaxel still leads to significant scar tissue and irreversible damage to the artery. ${ }^{7}$

Despite the downfalls associated with Paclitaxel, the drug-eluting balloon is still an effective design for drug delivery to vascular calcification. A gel coating could be employed to release a specific drug at a predetermined concentration slowly. The gel would need to be soluble, non-toxic, biocompatible, and able to bind to hydroxyapatite $\left(\mathrm{Ca}_{5}\left(\mathrm{PO}_{4}\right)_{3}\right)(\mathrm{HA})$, the main component of calcification in the arteries. ${ }^{8}$ Dextran-sulfate sodium salt is a previously used gel base that effectively covers these categories. ${ }^{9}$ Dextran is soluble in water and nontoxic, making it a viable option. Due to its hydrophilic nature, enhanced when combined with sulfate, dextran was studied for use as a delivery mechanism for hydrophobic, therapeutic drugs in the body.,10 It has shown success as a microsphere for drug delivery without losing important cargo before it reaches its desired location within the body. Clinically, it has been used as a therapeutic agent in various ways, including increasing plasma expansion and peripheral blood flow. ${ }^{11}$ At a low molecular weight, it has a typical half-life of eight hours, whereas its higher molecular weight counterparts exhibit an even longer half-life, making it an ideal vehicle for slow release. ${ }^{11,12}$ Dextran-sulfate has been previously used to form nanospheres loaded with insulin for delivery. ${ }^{13}$ As a hydrogel, it has been studied extensively as a delivery mechanism, with water 
content playing a significant role in the stability of the gel. ${ }^{14}$ In this study, we aim to show how this dextran-based gel is suitable for anti-calcification agent delivery due to its ability to bind to calcium deposits and withstand the shear force induced by blood flow.

The RANK/RANKL/OPG pathway is an important consideration in choosing a drug to load into the gel for delivery. While the pathway is mostly understood according to current research, conflicting reports on its effects in bone versus vasculature make it difficult to determine its exact mechanism of action. It is known that increased serum osteoprotegerin (OPG) is related to a decrease in bone loss and osteoclast activation in the skeletal system. ${ }^{15}$ OPG blocks RANK receptors on monocytes and macrophages in bone and prevents their differentiation into bone-resorbing bodies. This is where the gene, RANKL, becomes important. It naturally binds to the RANK receptors when uninhibited by OPG and initiates the differentiation process. RANKL upregulation is associated with an increase in osteoclastogenesis, which initiates the formation of osteoclasts. These cells then begin remodeling bone, leading to an increased bone loss. ${ }^{16,17} \mathrm{It}$ is hypothesized that RANKL could be introduced to the vasculature to activate monocytes and macrophages, causing differentiation into osteoclasts to resorb the plaque present in the arterial wall.

However, it is important to note that further exploration of the relationship of OPG and RANKL expression in the vascular system reveals that RANKL may be associated with plaque rupture in unstable coronary artery disease. ${ }^{18}$ Similarly, OPG, which plays a role in blocking osteoclastogenesis in bone, has been theorized to play a protective role against vascular calcification. ${ }^{19}$ In a study where OPG was knocked down in transgenic mice, an increased onset of vascular calcification was observed. ${ }^{19}$ In a different study measuring levels of OPG and RANKL in uremic mice, it was found that increased expression of RANKL was correlated with plaque accumulation in the arteries and increased observation of vascular calcification. ${ }^{20}$

This paradoxical relationship of OPG and RANKL to bone resorption is poorly understood. It is well known that vascular calcification and bone resorption are linked; an increase in bone loss causes an increase in bone formation in the arteries, and the opposite is true. The same factors that decrease bone mass in the skeletal system appear to increase bone mass in the arteries. As previously mentioned, this has been shown through the upregulation of RANKL in vascular calcification. ${ }^{18}$ However, there are two potential explanations for this issue. First, it is important to recognize that RANKL is currently viewed as a biomarker rather than a mediator. Increased RANKL is triggered by the aggregation of inflammatory molecules and cytokines in order to maintain balance through an immune response. ${ }^{21}$ Because inflammatory molecules are recruited during calcification; it is natural to see an increase in the recruitment of RANKL to help ameliorate this issue. This could account for the increase in RANKL expression.

Secondly, OPG is found in two primary locations: both in the serum and the plasma. High concentrations in each can lead to significantly different outcomes. An increase in serum OPG is reported to lead to bone destabilization and breakdown, while an increase in plasma OPG is associated with increased atherosclerosis. ${ }^{21}$ Fibrinogen, a clotting factor found in plasma, could be a component at play in this observation, meaning that the location of delivery is a determining factor in the effects of the gene.

Through this investigation, we believe that RANKL has the potential to reverse arterial calcification if delivered directly to the site of injury using the modified dextran-sulfate gel model. ${ }^{9}$ To further explore this potential treatment, our first goal was to determine the effectiveness of dextran-sulfate as a gel vehicle for RANKL delivery by using the protein bovine serum albumin (BSA). BSA was used as a substitute for RANKL because they are both soluble and have similar molecular weights (BSA averaging $\sim 50-60 \mathrm{kDa}$ and RANKL $\sim 60-70 \mathrm{kDa}$ ) (Sigma). ${ }^{22}$ These molecular weights, while not exact, are similar enough to validate using BSA as a RANKL surrogate. BSA is soluble in ethanol and glycerol, making it suitable for testing purposes. Finally, it is a readily available substitute that can be measured using the described methods. In this study, we looked at two separate aspects of the gel: 1) its ability to effectively bind to HA to determine whether it would stay in place once delivered to calcification and 2) its ability to withstand the shear forces incurred by blood flow in the artery without completely dissolving before drug (RANKL) could be delivered. Here, we use the dextran-sulfate based gel cast on nylon and incorporate BSA as a substitute for RANKL. With the use of IR spectrometry, SEM and EDS, and shear flow testing, we examined binding properties. Our results indicate future potential success for this application.

\section{METHODS AND PROCEDURES}

All materials were purchased from Sigma Aldrich unless otherwise noted.

\section{Gel Formation}

The gel formation protocol was developed as a modified model from Lamichhane. ${ }^{9}$ In short, $1.875 \mathrm{~g}$ of dextran sodium sulfate salt, $2.25 \mathrm{~mL}$ distilled water, and $0.38 \mathrm{~mL}$ glycerin were combined on a stir plate at 200 RPM for three hours. If no BSA was used, $1 \mathrm{~mL}$ of ethanol was added to the mixture while on the stir plate. For experiments loaded with BSA, $65.55 \mathrm{mg}$ BSA was added to the mixture while on the stir plate, allowed to dissolve, and then followed by $1 \mathrm{~mL}$ of ethanol. The gel continued to stir for 30 minutes at room temperature. Once combined, the gel was spread evenly in a thick layer (0.1-0.5 mm thick) over either a thin sheet of sterile nylon (purchased from VWR) or catheter tubing (donated by Oktibbeha County Hospital), dependent on testing. 
Gels were cross-linked to increase surface stability and lifespan. To do so, gels were dipped in a 1:9 ratio of glycerin to ethanol mixture for $30 \mathrm{~s}$ each followed by dipping in 100\% ethanol for one minute. A setting period of 24 hours elapsed for each gel before additional testing to allow gel time to dry.

\section{Hydroxyapatite Coating.}

To determine binding ability to HA, gels formed on nylon sheets $(2 \mathrm{~cm} \times 2 \mathrm{~cm})$ were placed in beakers with $1 \mathrm{X}$ Phosphate Buffered Saline (PBS) and $15 \mu \mathrm{M} \mathrm{HA}$ in a $37^{\circ} \mathrm{C}$ shaker to mimic in vivo conditions. Gels soaked for either one hour, four hours, or 24 hours with agitation. Gels were then rinsed in fresh 1X PBS and left to dry for a minimum of 24 hours before testing.

\section{IR Spectroscopy}

The gel samples previously soaked in HA for one hour, four hours, and 24 hours were tested with infrared spectroscopy (IR) using MicroLab technology to measure absorbance and transmittance of the sample. A control sample, dextran gel that was not soaked in the HA/PBS mixture, was tested for baseline reference, as well as pure powdered HA. All samples soaked in the HA/PBS mixture were dried for at least 24 hours to ensure that no water was left in the sample to affect the IR reading. For sample preparation, the gel was carefully removed from nylon after being dried, then directly transferred to the crystal for analysis. After the machine-generated graphs for each sample, peaks were analyzed and classified using a standard table of wavelengths.

\section{Scanning Electron Microscopy/Energy Dispersive Spectroscopy}

The same gel sample preparation procedure previously described for IR analysis (gels soaked in HA for one hour, four hours, and 24 hours) was also used to prepare samples for analysis with SEM and EDS testing. Small squares $(1 \mathrm{~cm} \mathrm{x} 1 \mathrm{~cm})$ were cut from the center of the gels on the nylon sheets to obtain the most representative sample. Samples were then mounted on a metal peg and coated with a $15 \mathrm{~nm}$ thick layer of platinum. A JEOL 6500F Field Emission SEM with an X-EDS spectrometer and Oxford Instruments INCAEnergy+ software was used for analysis.

\section{Shear Testing}

An expired standard hospital catheter $(4.36 \mathrm{~mm}$ diameter) was modified for use in recapitulating blood flow through an artery. Two experimental designs were used to test the adhesion of BSA to the gel. Dulbecco's Modified Eagle's Medium (DMEM) was used to mimic blood, as is standard in blood shear flow testing. A syringe pump (Fisher Scientific) was paired with a syringe containing DMEM, as shown in Figure 1. Catheter tubing was cut to $10 \mathrm{~cm}$, and the gel was placed opposite of end connected to the syringe. A flow rate of $956.9 \mathrm{~mL} / \mathrm{hr}$ created a shear force on the wall of the tubing as well as the catheter surface. The flow rate was chosen based on a published model of in vitro blood flow (see Discussion). ${ }^{23}$ Runoff was collected in a $100 \mathrm{~mL}$ beaker and briefly disturbed by shaking before aliquots were taken to ensure even sample collection. Aliquots of $1 \mathrm{~mL}$ were taken at the following time points for BSA analysis: 30 seconds and every minute for six minutes. This process was repeated twice.

The process was repeated a third time with an alternative design. The same tubing was used; however, a coated catheter was not used. Instead, a microscope slide with a small square sheet of nylon coated with the gel was placed diagonally into a beaker, and the same flow rate and syringe set-up were used as outlined previously. Aliquots of $1 \mathrm{~mL}$ were taken at the following time points for BSA analysis: 30 seconds, and every minute for six minutes.

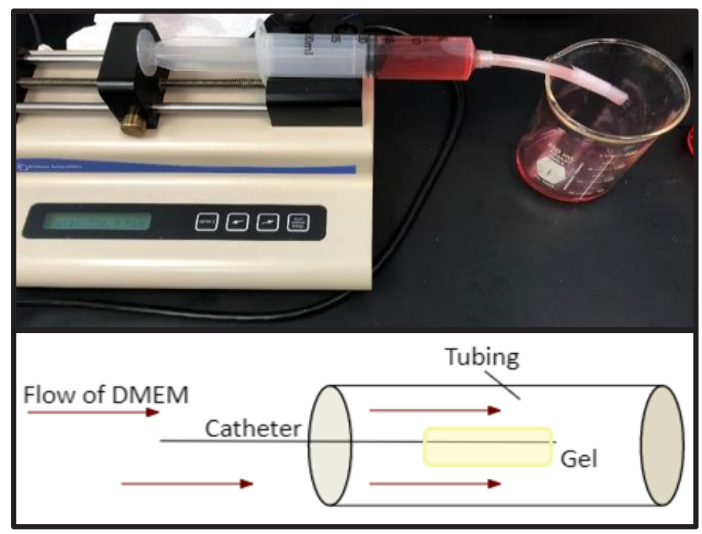

Figure 1. The shear flow protocol is illustrated where the gel coated catheter is fed through plastic tubing and connecting to a syringe pump. 


\section{BCA Assay}

A bicinchoninic acid (BCA) assay was used to determine the concentration of protein sheared off during the shear testing. Reagents were prepared according to the kit (Thermofisher). In a standard 96-well plate, $50 \mu \mathrm{L}$ was transferred from each time point aliquot in triplicate. A standard concentration curve was established, and the 96-well plate was read using a spectrophotometer. Results were analyzed by comparing samples to the standard curve.

\section{RESULTS}

Binding Assessment

IR produced spectra (transmittance vs. wavelength) that provided information about chemical bonds. Two control samples, dextran gel only, without being soaked in HA/PBS mixture, and HA only, in powdered form, were read. As previously mentioned, samples were prepared by removing the cast gel from the nylon after soaking in HA/PBS for the allotted time, dried for 24 hours, and placed directly on the machine's crystal for analysis. In Figure 2 depicting IR results from all samples, the control tests revealed an aromatic carbon-carbon double bond visible around $1600 \mathrm{~cm}^{-1}$, from the ring characteristic of dextran, as well as carbon-hydrogen bonds appearing in the $2900 \mathrm{~cm}^{-1}$ region. No peak occurs at $1200 \mathrm{~cm}^{-1}$. In the HA only test, the presence of a potential phosphate $\left(\mathrm{PO}_{4}\right)$ bond appears at $500 \mathrm{~cm}^{-1}$. No peaks occur at the $1200 \mathrm{~cm}^{-1}$ and $3300 \mathrm{~cm}^{-1}$ wavelengths.

A sample from each of the PBS/HA soak time points (one hour, four hours, and 24 hours) was tested using IR. A small amount of gel remained at the four hour and 24-hour time points. For the one hour, dip-coated gel, peaks can be seen starting to form at $1200 \mathrm{~cm}^{-1}$ and $3300 \mathrm{~cm}^{-1}$. For the four hour PBS/HA soak of the dip-coated gel, peaks can be observed surrounding the $500 \mathrm{~cm}^{-1}$ and around the $1500 \mathrm{~cm}^{-1}$ region. Additionally, peaks can be seen beginning to form around the $3300 \mathrm{~cm}^{-1} \mathrm{mark}$. A notable peak appears around the $500 \mathrm{~cm}^{-1}$ mark. A peak can be seen beginning to form at the $1200 \mathrm{~cm}^{-1}$ mark.

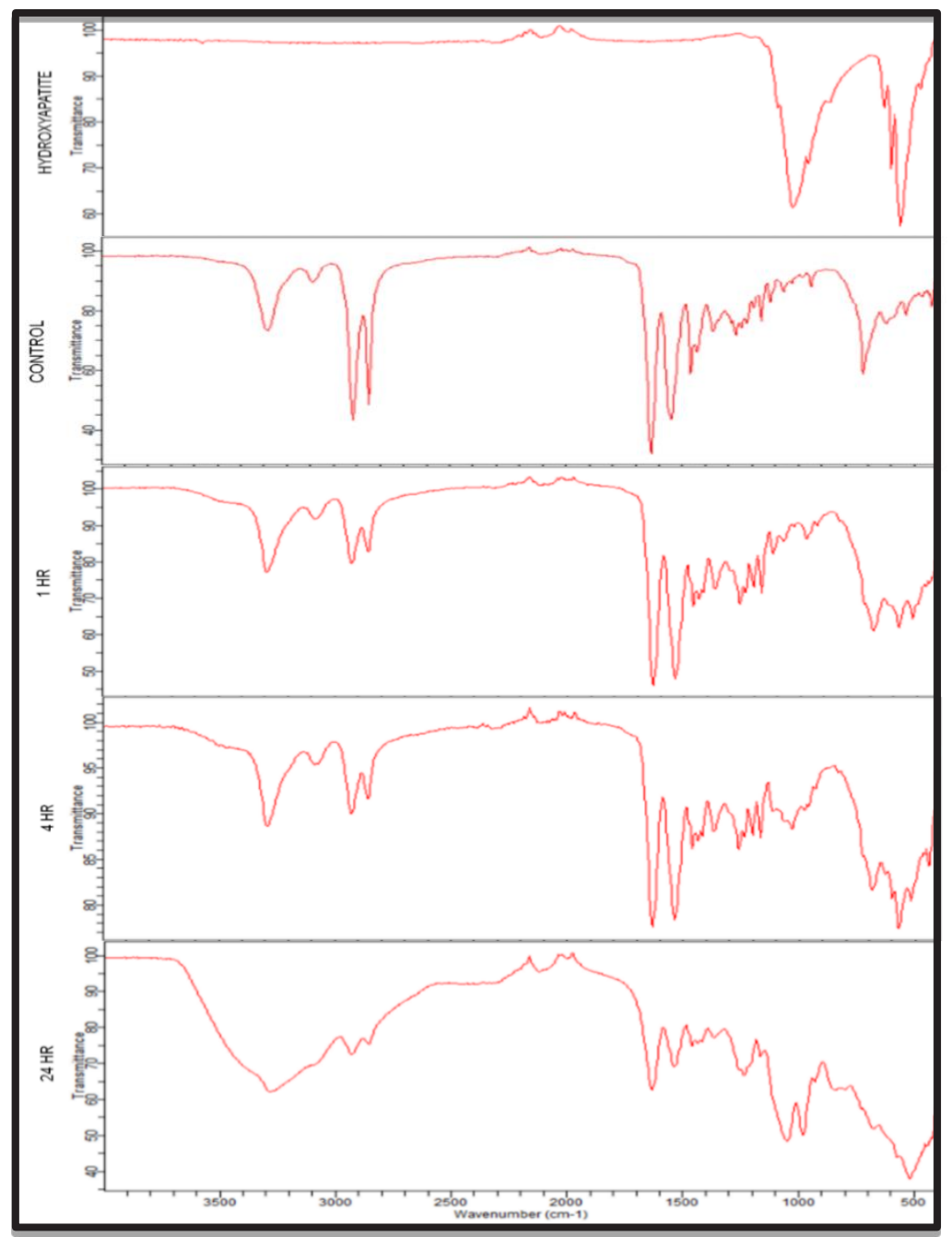

Figure 2. IR spectra results for $\mathrm{HA}$ only, control, one, four, and 24-hour gels. Increase in intensity at $1200 \mathrm{~cm}^{-1}$ indicates $\mathrm{Ca}$ binding to $\mathrm{SO}_{4}$. 


\section{Shear Force Assessment}

A BCA assay revealed the protein concentration that was released from the gel on the catheter during shear force testing. The assay was completed in triplicate, and the average value for each of the three trials was averaged together. Effectively no protein (BSA) was sheared off between the zero minute and five-minute time points. Figure 3 depicts the concentration of protein in $\mu \mathrm{g} / \mathrm{mL}$ in the media collected for aliquots at each time point (minutes). Between the five to six minute time period, an average of $0.029 \pm 0.024 \mu \mathrm{g} / \mathrm{mL}$ was released from the gel.

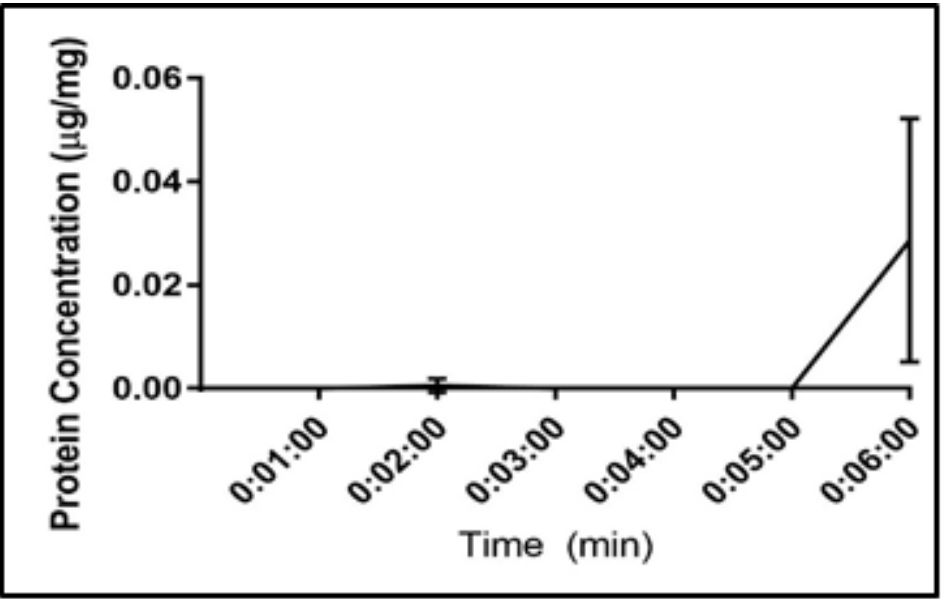

Figure 3. The graph above illustrates the results from shear testing, with $0.55 \pm 1.3 \mathrm{ng} / \mathrm{mL}$ released at two minutes, and $0.029 \pm 0.024 \mu \mathrm{g} / \mathrm{mL}$ released from five to six minutes.

When the end of the catheter was coated in BSA loaded gel and used for shear flow testing, minimal protein was lost between the zero and one minute mark. Similar results were seen with the slant test as described above, in which $0.1184 \pm 0.049 \mu \mathrm{g} / \mathrm{mL} \mathrm{of}$ protein was found in collected media at the five to six-minute mark.

\section{Scanning Electron Microscopy (SEM)/Energy Dispersive Spectrometry (EDS) Results}

The one hour sample produced definitive results, shown in Figure 4, which calcium was indeed found on the surface of the gel. After soaking one hour in PBS/HA, calcium appeared on the sample at $0.45 \%$ by weight, with the images shown below taken at the $100 \mu \mathrm{m}$ scale. The four hour and 24-hour samples produced negligible calcium content by EDS measurement.
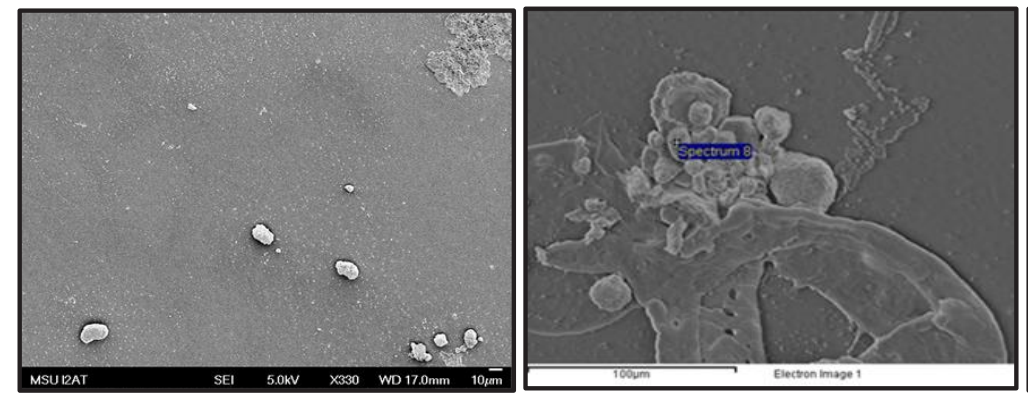

\begin{tabular}{|c|c|c|}
\hline Element & Weight\% & Atomic\% \\
\hline $\mathrm{C}$ & 82.58 & 87.69 \\
\hline $\mathrm{O}$ & 13.88 & 11.07 \\
\hline $\mathrm{P}$ & 0.15 & 0.06 \\
\hline $\mathrm{Ca}$ & 0.45 & 0.14 \\
\hline
\end{tabular}

Figure 4. EDS testing revealed that the calcium on the surface of the gel was $0.45 \%$ by weight.

\section{DISCUSSION}

\section{Design criteria}

While current treatments are available to help reduce the effect of calcification on blood flow, no treatment completely eradicates vascular calcification. As a result, subsequent interventions are needed. The concept for these constructs is founded upon integral components that work in tandem: a gel coated balloon angioplasty procedure that can deliver anti-calcification agents to the vessel. The gel coating should detach from the balloon and adhere to calcification in order to time-release a concentration of RANKL. Once applied to the site of vascular calcification, the RANKL should combat the hydroxyapatite buildup by causing macrophage differentiation into osteoclasts to resorb bone tissue. 
The gel coating requirements included the following: non-toxicity, biocompatibility, cost-effectiveness, and stability in aqueous environments, ability to bind to hydroxyapatite and carry a therapeutic drug to the site of calcification. Previous use of dextran-sulfate gel has also confirmed its hydrophilic and biocompatible properties make it useful as a drug delivery mechanism. ${ }^{9}$ However, with our results, we can show that it can bind in the desired manner to sites of calcification where RANKL delivery will be most useful. We also show that it can withstand high shear forces without losing loaded protein too quickly.

\section{Comparison of gel-to-nylon coating methods}

Two coating methods were tested in the early stages of this experiment: spin-coating and dip-coating. The dip-coating method on the thin nylon film pieces is commonly used with sol-gels and allowed for a thicker layer of gel to be formed. ${ }^{24}$ Conversely, spincoating resulted in a thin, even layer on nylon. After the mixture of glycerol and ethanol was used to cross-link the spun gel, it dissolved nearly instantaneously, too quickly for practical purposes. As well, if this design were to be mass-produced, a dip coating may be a more cost-effective approach, as no specialized machinery would be required. Sol-gel dip-coating methods have been reported as inexpensive and easier to employ than spray or sputter-coatings. ${ }^{24-26}$ Because the dip-coating method can be applied independent of the shape of the object being coated, it allows for a uniform coating. ${ }^{25}$

\section{Roles of dextran, BSA, and bydroxyapatite}

Dextran-sulfate sodium salt provides a non-toxic, water-soluble, and relatively inexpensive vehicle for drug delivery. ${ }^{10}$ For preliminary testing purposes, BSA was chosen to represent RANKL because of its similarity in molecular weight. Our design involves targeting and attachment to sites of arterial calcification. To model this in vitro, the main component of these calcium aggregates, HA, was used to assess the gel's targeted binding capabilities. To our knowledge, dextran-sulfate has not been previously studied concerning binding to either bone material or hydroxyapatite, though multiple studies have examined hydrogels as potential scaffolds for bone regeneration. ${ }^{27,28}$ Many gels have also been functionalized with different peptides in previous experiments to be used as bone defect fillers. ${ }^{29}$

\section{IR Testing}

The most significant results achieved were those at the one-hour PBS/HA soak time point. Referring to Figure 5, peaks can be seen starting to form at $1200 \mathrm{~cm}^{-1}$, indicating calcium binding to sulfate. This signifies that the dextran-based gel has successfully bound to HA. Additionally, the peak at $3300 \mathrm{~cm}^{-1}$ corresponds with hydroxyl $(-\mathrm{OH})$ groups present after binding. Peaks at approximately $1000 \mathrm{~cm}^{-1}$ are characteristic of HA. Intensity decreases at the $2900 \mathrm{~cm}^{-1}$ peak (C-H bond) which could indicate the loss of $\mathrm{C}-\mathrm{H}$ bonds where $\mathrm{Ca}_{3}\left(\mathrm{PO}_{4}\right)_{2}$ is binding.

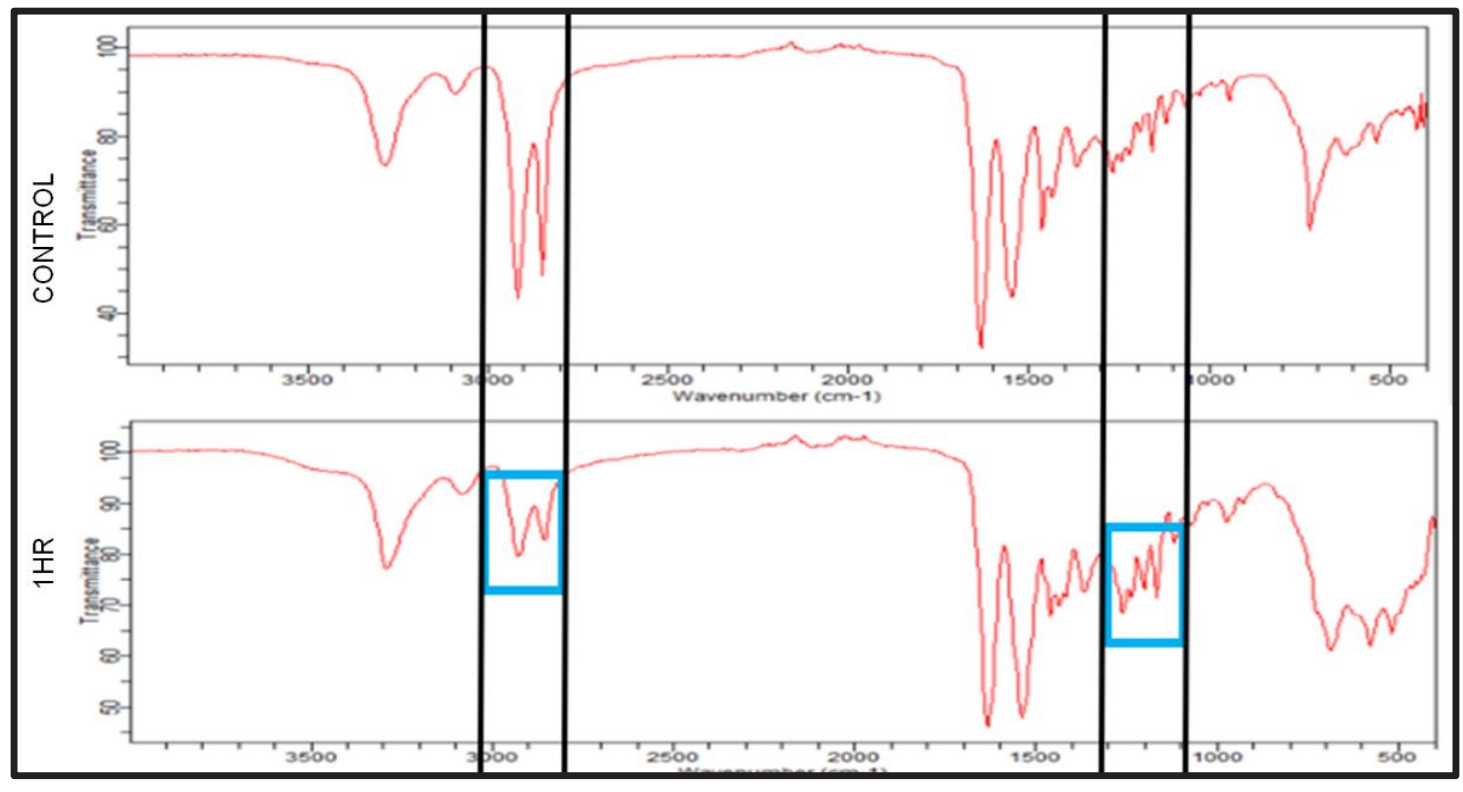

Figure 5. After one hour, a peak forms at $1200 \mathrm{~cm}^{-1}$, indicating calcium binding with sulfate (HA to gel). There is a loss of intensity at $2900 \mathrm{~cm}-1$ (C-H bond) that could indicate the loss of $\mathrm{C}-\mathrm{H}$ bonds where $\mathrm{Ca}_{3}\left(\mathrm{PO}_{4}\right)_{2}$ is binding 


\section{SEM/EDS Testing}

Preliminary SEM and EDS results in Figure $\mathbf{6}$ provide evidence that calcium was present on the dip-coated sample that was soaked in the HA/PBS solution for one hour. Ideally, this means that the gel can adhere to calcification for at least one hour. However, the one and 24-hour samples show no signs of calcium still on the surface of the gel. This is potentially the result of the gel dissolving over the longer period of time in solution. From this, it is hypothesized that the gel would be able to bind to the calcium deposits for the duration of time needed for the balloon to be inflated, removed, and then allow for the release of RANKL at the site. Further testing is required to confirm this hypothesis.
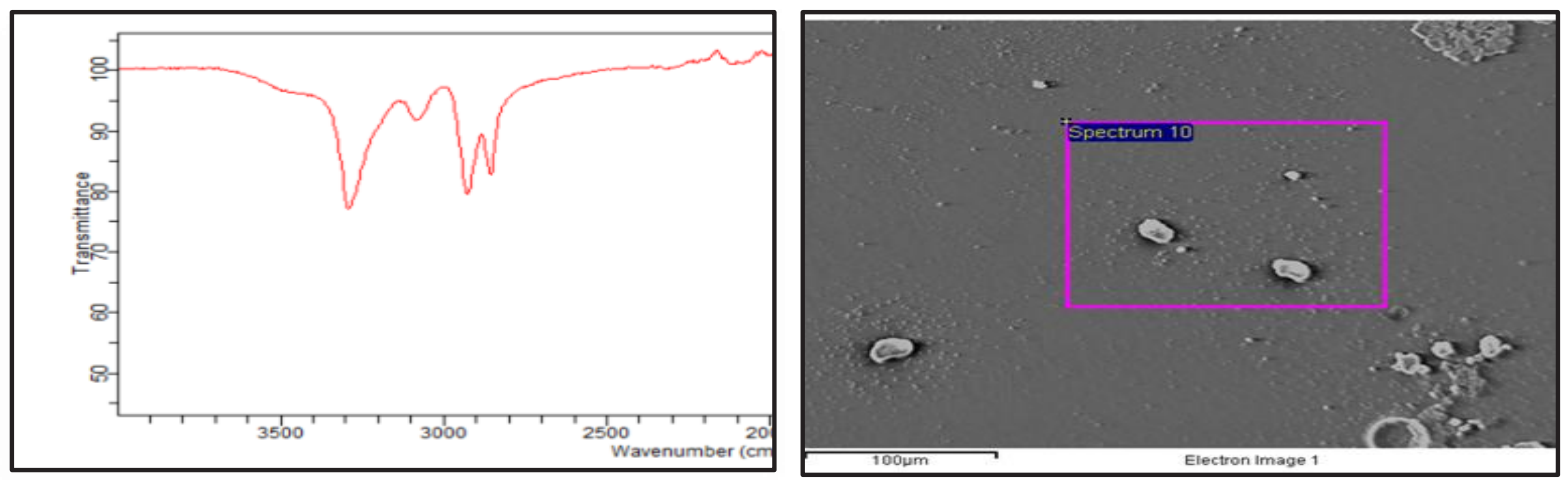

Figure 6. IR for the one-hour soaked gel confirmed through SEM imaging with evidence of Calcium bound to the gel surface.

\section{Shear Testing}

Though blood flow and shear stress on arterial walls proves a contentious topic in bio-fluid mechanics, a previous study shows a validated computational model with a flow rate of $19.4 \mathrm{~mL} / \mathrm{min}$ (or 1,164 mL/hr). ${ }^{23}$ This number was decided on using a pulsatile flow equation, shown in Equation 1 below using a dynamic blood viscosity of $1.5 \mathrm{cP}$. Because DMEM has a lower viscosity (1.1 $\mathrm{cP}$ ), a lower speed is required to simulate blood flow across the same cross-sectional area. The accepted blood flow rate according to Engel et al. is $19.4 \mathrm{~mL} / \mathrm{min}^{23}$ The max speed on the machine used was $\sim 16 \mathrm{~mL} / \mathrm{min}$ and was therefore used for shear testing.

$$
u(r, t)=\operatorname{Real}\left(\sum_{\omega=1}^{\infty} \frac{\widehat{q}}{\pi R^{2}}\left(\frac{\left(1-\frac{J_{0}\left(\alpha \frac{r}{R} i^{3 / 2}\right)}{J_{0}\left(\alpha i^{3 / 2}\right)}\right)}{\left(1-\frac{2 J_{1}\left(\alpha i^{3 / 2}\right)}{\alpha i^{3 / 2} J_{0}\left(\alpha i^{3 / 2}\right)}\right)}\right) e^{i \omega t}\right),
$$

Equation 1.

While it is difficult to narrow down a specific value for blood flow through an artery, for this preliminary study, a rate between 15$20 \mathrm{~mL} /$ min most accurately reflects what can be done outside of in vivo testing according to literature. ${ }^{30}$ The speed is large enough to simulate the transitional blood flow moving through a vessel with a diameter of three to five $\mathrm{mm}$.

From the quantitative measurement of protein in shared media, the dextran-based gel appears to be successful regarding a delivery vehicle for anti-calcification agents. Ideally, the gel should hold the medication or drug long enough for the balloon to reach the site of calcification in the arteries. According to reports on angioplasty procedures, the standard time for a surgeon to deliver the balloon is three to five minutes. ${ }^{31}$ From shear testing, it was found that little to no protein was lost in the first five minutes of shear flow over the gel. Once the five-minute mark was reached, minimal amounts of BSA appeared in the collected media. This is indicative of proper release time in terms of the gel dissolving to allow RANKL to bind to calcification. However, further testing is required to ensure that RANKL would behave similarly. In previous studies, RANKL and fluorescent dextran were incubated together with ST2 cells and were shown to co-localize together in the same regions within the cells, indicating that they may have similar binding affinities. ${ }^{32}$

\section{CONCLUSIONS}

While a drug-eluting stent is already available in commercial use, it is not wholly effective due to design elements and drug choice. The current drug is Paclitaxel; however, it is not an adequate countermeasure to calcification and staunches the magnitude of the impacts. ${ }^{7}$ While future work is required to explore the success of RANKL over Paclitaxel, the current experimentation sought to 
determine whether a dextran-based gel could effectively bind to calcification sites and deliver RANKL. Three distinct assertions were formed in investigating the gel's properties. Dextran-sulfate sodium salt lent a satisfactory outcome as a binding agent. The gel was able to endure the hydroxyapatite soak treatments. The dextran-sulfate and HA were able to adhere to one another, signifying favorable attachment. The dextran-sulfate gel was able to withstand the in vivo shear forces of the blood that it would encounter during the surgical procedure of balloon angioplasty. Future implications for the work done indicate the need for further testing at a more complex level. This would involve replacing BSA with RANKL to determine if it exhibits similar behavior. Explicitly, a re-evaluation would be beneficial to solidify that the conclusions drawn from the research project are accredited. The outcomes from the SEM and EDS supplied qualitative answers and confirmed the IR results. However, a verification of these experimental developments is necessary. As well, a co-culture involving osteoclast progenitor cells in the presence of the gel would allow observation of whether or not cells will both survive and differentiate in the presence of the drug. An in vivo assessment of shear flow is also required to prove the blood flow model presently used accurately reflects working conditions and to give evidence to the overall success of the design.

\section{ACKNOWLEDGMENTS}

Authors thank Dr. Yu in the Agricultural and Biological Engineering Department at Mississippi State University for guidance throughout the project. Authors also thank Dr. Scott and Guerry Sanchez in the chemistry department for the use of IR instrument. Authors also thank the previous senior design group, notably Christian Griffith and Rachel Hybart for their preliminary research and guidance.

\section{REFERENCES}

1. Benjamin, EJ., Blaha, MJ., Chiuve, SE., Cushman, M., Das, SR., Deo, R., de Ferranti, SD., Floyd, J., Fornage, M., Gillespie, C., Isasi, CR., Jimenez, MC., Jordan, LC., Judd, SE., Lackland, D., Lichtman, JH., Lisabeth, L., Liu, S., Longenecker, CT., Mackey, RH., Matsushita, K., Mozaffarian, D., Mussolino, ME., Nasir, K., Neumar, RW., Palaniappan, L., Pandey, DK., Thiagarajan, RR., Reeves, MJ., Ritchey, M., Rodriguez, CJ., Roth, GA., Rosamond, WD., Sasson, C., Towfighi, A., Tsao, CW., Turner, MB., Virani, SS., Voeks, JH., Willey, JZ., Wilkins, JT., Wu, JHY., Alger, HM., Wong, SS., Muntner, P. (2017). Heart Disease and Stroke Statistics 2017 At-a-Glance. Circulation, n.d. bttps:// healthmetrics.heart.org/wpcontent/ uploads/2017/06/_Heart-Disease-and-Stroke-Statistics-2017-ucm_491265.pdf

2. Lee, KM., Lee, EO., Lee, YR., Joo, HK., Park, MS., Kim, C., Choi, S., Jeong, JO., and Jeon, BH. (2017). APE1/Ref-1 Inhibits Phosphate-Induced Calcification and Osteoblastic Phenotype Changes in Vascular Smooth Muscle Cells. IJMS, 18(10), 2053. https:// doi.org/10.3390/ijms18102053

3. Torremadé, N., Bozic, M., Panizo, S., Barrio-Vazquez, S., Fernandez-Martín, JL., Encinas, M., Goltzman, D., Arcidiacono, MV., Fernandez, E., Valdivielso, JM. (2016). Vascular Calcification Induced by Chronic Kidney Disease Is Mediated by an Increase of $1 \alpha$-Hydroxylase Expression in Vascular Smooth Muscle Cells. Journal of Bone and Mineral Research. bttps:// doi.org/10.1002/jbmr.2852

4. Redfors, B., Maehara, A., Witzenbichler, B., Weisz, G., Stuckey, TD., Henry, TD., McAndrew, T., Mehran, R., Kirtane, AJ., Stone, GW., Généreux, P. (2017). Outcomes After Successful Percutaneous Coronary Intervention of Calcified Lesions Using Rotational Atherectomy, Cutting Balloon Angioplasty, or Balloon-Only Angioplasty Before Drug-Eluting Stent Implantation. J of Invasive Cardiol, 11, 378-386.

5. Medtronic. Coronary Balloons, (2011). http:// www.medtronic.com/us-en/healthcare-professionals/products/cardiovascular/coronaryballoons.html (accessed January 2018)

6. Iqbal, J., Gunn, J., Serruys, PW. (2013). Coronary stents: historical development, current status, and future directions. British Medical Bulletin, 106(1), 193-211. bttps:/ / doi.org/10.1093/ bmb/ldt009

7. Cohen, JL., Glickstein, JS., Crystal, MA. (2017). Drug-Coated Balloon Angioplasty: A Novel Treatment for Pulmonary Artery In-Stent Stenosis in a Patient with Williams Syndrome. Pediatric Cardiology, 38(8), 1716-1721. https:// doi.org/10.1007/s00246017-1646-1

8. Richards, JM., Kunitake, J., Hunt, HB., Wnorowski, AN., Lin, DW., Boskey, AL., Donnelly, E., Estroff, LA., Butcher, JT.. (2018). Crystallinity of hydroxyapatite drives myofibroblastic activation and calcification in aortic valves. Acta Biomaterialia. 71, 24-36. bttps:// doi.org/10.1016/j.actbio.2018.02.024

9. Lamichhane, S., Anderson, J., Remund, T., Kelly, P., Mani., G. (2016). Dextran sulfate as a drug delivery platform for drugcoated balloons: Preparation, characterization, in vitro drug elution, and smooth muscle cell response. Journal of Biomedical Materials Research. Part B, Applied Biomaterials, 104(7), 1416-30. bttps:/ / doi.org/10.1002/jbm.b.33494

10. Lin, W. C., Yu, D. G., \& Yang, M. C. (2005). pH-sensitive polyelectrolyte complex gel microspheres composed of chitosan/sodium tripolyphosphate/dextran sulfate: Swelling kinetics and drug delivery properties. Colloids and Surfaces B: Biointerfaces, 44(2-3), 143-151. bttps:/ / doi.org/10.1016/j.colsurfb.2005.06.010

11. Van Tomme, S. R., \& Hennink, W. E. (2007). Biodegradable dextran hydrogels for protein delivery applications. Expert Revien of Medical Devices. https:// doi.org/10.1586/17434440.4.2.147 
12. Arturson, G., \& Wallenius, G. (1964). The intravascular persistence of dextran of different molecular sizes in normal humans. Scandinavian Journal of Clinical and Laboratory Investigation, 16(1), 76-80. https:/ / doi.org/10.3109/00365516409060485

13. Sarmento, B., Ribeiro, A., Veiga, F., \& Ferreira, D. (2006). Development and characterization of new insulin containing polysaccharide nanoparticles. Colloids and Surfaces B: Biointerfaces, 53(2), 193-202. bttps:// doi.org/10.1016/j.colsurfb.2006.09.012

14. Van Tomme, S. R., Van Steenbergen, M. J., De Smedt, S. C., Van Nostrum, C. F., \& Hennink, W. E. (2005). Self-gelling hydrogels based on oppositely charged dextran microspheres. Biomaterials, 26(14), $2129-2135$. https:// doi.org/10.1016/j.biomaterials.2004.05.035

15. Hofbauer, LC., Brueck, CC., Shanahan, CM., Schoppet, C., Dobnig, H. (2007). Vascular calcification and osteoporosis--from clinical observation towards molecular understanding. Osteoporosis International: A Journal Established as Result of Cooperation between the European Foundation for Osteoporosis and the National Osteoporosis Foundation of the USA, 18(3), 251-9. bttps: / / doi.org/ 10.1007/s00198-006-0282-z

16. Hofbauer, LC., \& Schoppet, M. (2004). Clinical implications of the osteoprotegerin/RANKL/RANK system for bone and vascular diseases. JAMA. https:// doi.org/10.1001/jama.292.4.490

17. Steeve, KT., Marc, P., Sandrine, T., Dominique, H., \& Yannick, F. (2004). IL-6, RANKL, TNF-alpha/IL-1: Interrelations in bone resorption pathophysiology. Cytokine and Growth Factor Reviews. https:/ / doi.org/ 10.1016/j.cytogfr.2003.10.005

18. Sandberg, WJ., Yndestad, A., Øie, E., Smith, C., Ueland, T., Ovchinnikova, O., Robertson, A., Müller, F., Semb, AG., Scholz, H., Andreassen, AK., Gullestad, L., Damås, JK., Frøland, SS., Hansson, GK., Halvorsen, B., Aukrust, P. (2006). Enhanced Tcell expression of RANK ligand in acute coronary syndrome: Possible role in plaque destabilization. Arteriosclerosis, Thrombosis, and Vascular Biology, 26(4), 857-863. https:/ / doi.org/10.1161/01.ATV.0000204334.48195.6a

19. Bucay, N., Sarosi, I., Dunstan, C. R., Morony, S., Tarpley, J., Capparelli, C., ... Simonet, W. S. (1998). Osteoprotegerin-deficient mice develop early onset osteoporosis and arterial calcification. Genes and Development, 12(9), 1260-1268. bttps://doi.org/10.1101/gad.12.9.1260

20. Panizo, S., Cardus, A., Encinas, M., Parisi, E., Valcheva, P., López-Ongil, S., Coll, B., Fernandez, E., Valdivielso, JM. (2009). RANKL increases vascular smooth muscle cell calcification through a rank-bmp4-dependent pathway. Circulation Research, 104(9), 1041-1048. https:/ / doi.org/10.1161/CIRCRESAHA.108.189001

21. Biscetti, F., Giovannini, S., Straface, G., Bertucci, F., Angelini, F., Porreca, C., Landolfi, R., Flex, A. (2016). RANK/RANKL/OPG pathway: genetic association with history of ischemic stroke in Italian population. Eur Rev Med Pharmacol Sci, 20(21), 4574-4580. bttp:/ / wmw.ncbi.nlm.nih.gov/pubmed/27874938

22. Khosla, S. (2001). Minireview: The OPG/RANKL/RANK system. Endocrinology. https://doi.org/10.1210/endo.142.12.8536

23. Engels, G. E., Blok, S. L. J., \& van Oeveren, W. (2016). In vitro blood flow model with physiological wall shear stress for hemocompatibility testing-An example of coronary stent testing. Biointerphases, 11(3), 31004. bttps://doi.org/10.1116/1.4958979

24. Asri, R. I. M., Harun, W. S. W., Hassan, M. A., Ghani, S. A. C., \& Buyong, Z. (2016). A review of hydroxyapatite-based coating techniques: Sol-gel and electrochemical depositions on biocompatible metals. Journal of the Mechanical Behavior of Biomedical Materials. https:// doi.org/10.1016/j.jmbbm.2015.11.031

25. Haddow, D. B., James, P. F., \& Van Noort, R. (1996). Characterization of sol-gel surfaces for biomedical applications. Journal of Materials Science: Materials in Medicine, 7(5), 255-260. https:/ / doi.org/10.1007/BF00058562

26. Gupta, R., \& Kumar, A. (2008). Bioactive materials for biomedical applications using sol-gel technology. Biomedical Materials (Bristol, England), 3(3), 34005-34020. https:// doi.org/10.1088/1748-6041/3/3/034005

27. Kim, J., Kim, I. S., Cho, T. H., Lee, K. B., Hwang, S. J., Tae, G., Noh, I., Lee, SH., Park, Y., Sun, K. (2007). Bone regeneration using hyaluronic acid-based hydrogel with bone morphogenic protein-2 and human mesenchymal stem cells. Biomaterials, 28(10), 1830-1837. https:// doi.org/10.1016/j.biomaterials.2006.11.050

28. Dalton, PD., \& Woodfi, T. (2008). Polymeric Scaffolds for Bone Tissue Engineering. Bone, 32(3), $2004-2005$. bttps:// doi.org/10.1002/jbm.a.31829.ELECTROSPINNING

29. Dennis, S. C., Whitlow, J., Detamore, M. S., Kieweg, S. L., \& Berkland, C. J. (2017). Hyaluronic-Acid-Hydroxyapatite Colloidal Gels Combined with Micronized Native ECM as Potential Bone Defect Fillers. Langmuir: The ACS Journal of Surfaces and Colloids, 33(1), 206-218. bttps://doi.org/10.1021/acs.langmuir.6b03529

30. Aarnoudse, W., van't Veer, M., Pijls, N. H. J., ter Woorst, J., Vercauteren, S., Tonino, P., ... van de Vosse, F. (2007). Direct Volumetric Blood Flow Measurement in Coronary Arteries by Thermodilution. Journal of the American College of Cardiology, 50(24), 2294-2304. https:/ / doi.org/ 10.1016/j.jacc.2007.08.047

31. Schorn, I., Malinoff, H., Anderson, S., Lecy, C., Wang, J., Giorgianni, J., \& Papandreou, G. (2017). The LUTONIX ${ }^{\circledR}$ drugcoated balloon: A novel drug delivery technology for the treatment of vascular disease. Advanced Drug Delivery Reviews. bttps:// doi.org/10.1016/j.addr.2017.05.015

32. Kariya, Y., Honma, M., Aoki, S., Chiba, A., \& Suzuki, H. (2009). Vps33a mediates RANKL storage in secretory lysosomes in osteoblastic cells. Journal of Bone and Mineral Research, 24(10), 1741-1752. https:/ / doi.org/10.1359/jbmr.090409 


\section{ABOUT STUDENT AUTHORS}

Jenna Mosier, Hannah Stealey, Kalifa Stringfield, and Katie Webb graduated in May of 2018 from Mississippi State University.

\section{PRESS SUMMARY}

Vascular calcification is a condition in which the arteries stiffen and plaque build-up occurs, blocking blood flow. Current treatments for this issue are only temporary solutions, with drug-eluting stents being the most successful. This experiment seeks to design a gel coating for stents that is safe and nontoxic within the body and can successfully carry and release anti-calcification drugs to the state of diseased arteries. From the results, it was determined that a stable and biocompatible gel was formed. 
Print Edition ISSN 1536-4585

Online Edition ISSN 2375-8732 